Florida International University FIU Digital Commons

$12-8-1997$

\title{
Development and experimental verification of a three-dimensional model of left ventricular flow dynamics
}

Tong Ding

Florida International University

DOI: $10.25148 /$ etd.FI14062294

Follow this and additional works at: https://digitalcommons.fiu.edu/etd

Part of the Mechanical Engineering Commons

\section{Recommended Citation}

Ding, Tong, "Development and experimental verification of a three-dimensional model of left ventricular flow dynamics" (1997). FIU Electronic Theses and Dissertations. 2822.

https://digitalcommons.fiu.edu/etd/2822 


\title{
FLORIDA INTERNATIONAL UNIVERSITY
}

Miami, Florida

DEVELOPMENT AND EXPERIMENTAL VERIFICATION OF A THREE-DIMENSIONAL MODEL OF LEFT VENTRICULAR FLOW

\author{
DYNAMICS
}

A thesis submitted in partial satisfaction of the

requirements for the degree of

MASTER OF SCIENCE

IN

MECHANICAL ENGINEERING

by

Tong Ding

1998 
To: Gordon Hopkins

College of Engineering \& Design

This thesis, written by Tong Ding, and entitled DEVELOPMENT AS WELL AS EXPERIMENTAL VERIFICATION OF A THREE-DIMENSIONAL MODEL OF LEFT VENTRICULAR FLOW DYNAMICS, having been approved in respect to style and intellectual content, is referred to you for judgement.

We have read this thesis and recommend that it be approved.

Dr. James Moore

Dr. Ceasar Levy

Dr. Richard T. Schoephoerster, Major Professor

Date of Defense: December 8, 1997

The thesis of Tong Ding is approved.

Dean Gordon Hopkins

College of Engineering \& Design

Dr. Richard L. Campbell

Dean of Graduate Studies

Florida International University, 1998 


\section{ACKNOWLEDGMENTS}

I wish to thank Dr. Levy and Dr. Moore for their helpful comments and patience. I also want to thank the members of the lab: Flavio Souza Campos, for his valuable advice and help; Lijun Niu, for her help with everything.

A special thanks must go to my major professor, Dr. Schoephoerster, for his support and encouraging comments, and especially for having the confidence in me to give me the chance to do this project. 


\section{ABSTRACT OF THE THESIS}

\section{DEVELOPMENT AND EXPERIMENTAL VERIFICATION OF A THREE-DIMENSIONAL MODEL OF LEFT VENTRICULAR FLOW DYNAMICS \\ by}

Tong Ding

Florida International University, 1998

\section{Professor Richard T. Schoephoerster, Major Professor}

Ischemic heart disease, which results from the insufficient coronary artery blood flow is a leading cause of mortality in developed countries. It manifests itself by abnormal left ventricular wall motion during systole.

A three dimensional numerical model was developed to simulate the flow patterns in the left ventricle. Numerical solutions were obtained by discretizing the Navier-Stokes equations for viscous, incompressible, steady flow using finite element method.

A diagnostic index Central Ejection Region (CER) as well as its quantitative version CER coefficient which are based on the flow patterns were defined as the region in which velocity vectors were aligned 5 degrees from the long axis. They seem to be very sensitive to the degrees and size of ischemia. 
To validate the numerical method, experimental measurements as well as the numerical computation were performed on sphere-shape normal and the ischemic left ventricle model. A good agreement has been achieved. 


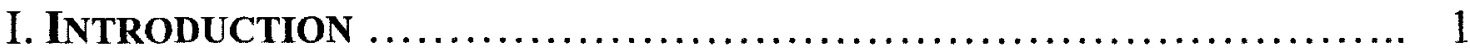

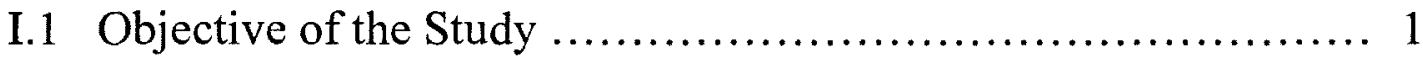

I.2.1 Physiology of Human Heart.................................. 3

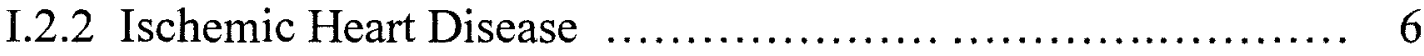

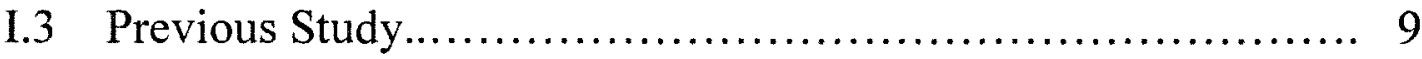

I.4 Problem Statement ....................................... 14

II. Mathematical Equations and Numerical Methodology.... 16

II.1. Problem Definition ................................... 16

II.2. Mathematical Governing Equations ....................... 17

II.3.1 Numerical Method ..................................... 18

II.3.2 Formulations of the Discrete Problem ....................... 18

II.4.1 Geometry Generation .................................. 20

II.4.2 Geometry Data Modification.............................. 23

II.4.3 Mesh Algorithm........................................ 24

II.5.1 Boundary Conditions ................................... 26

II.5.2 Simulated Ischemic Boundary Conditions ................... 27

II.6.1 Central Ejection Region and CER Coefficient ................ 31

II.6.2 Data Processing ......................................... 33

III EXPERIMENTAL VERIFICATION OF THE NUMERICAL METHOD ...... 35

III.1 Experimental Setup ...................................... 35

III.2 Digital Particle Image Velocimetry System ................... 37

III.3 Experiment Procedure ..................................... 39

III.4 Geometry Reconstruction ................................ 41

IV RESULTS AND DISCUSSION .................................... 43

IV.1 Comparison of the Numerical and Experimental Results ...... 43 IV.2.1 Flow Patters for Various Time Steps of Normal and Ischemic Left Ventricles .............................................. 45

IV.2.2 Flow Pattern of Simulated Ischemic Left Ventricles .......... 47 IV.3.1 CER and CER Coefficient of Normal and Ischemic Left Ventricles 
IV.3.2 CER and CER Coefficient of Simulated Ischemic Left Ventricles 51

IV.4 Discussion ........................................ 54

V CONCLUSIONS AND RECOMMENDATIONS FOR THE FUTURE WORK... 98 V.1 Conclusion ............................................ 98

V.2 Recommendations for Future Work ....................... 100

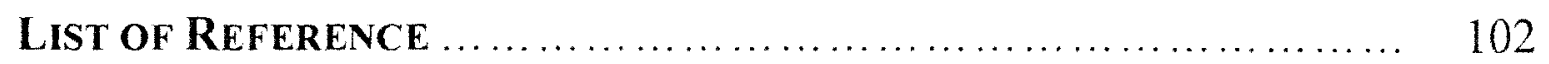

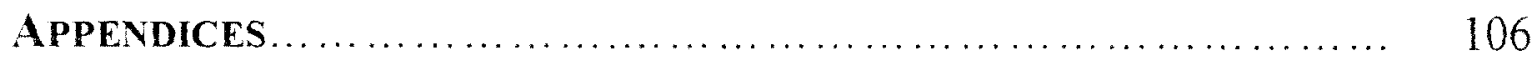




\section{LIST OF FIGURES}

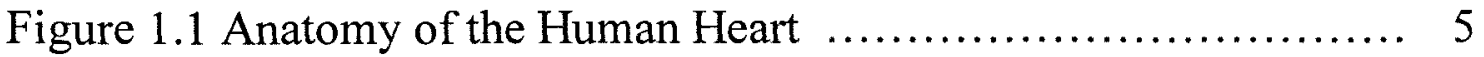

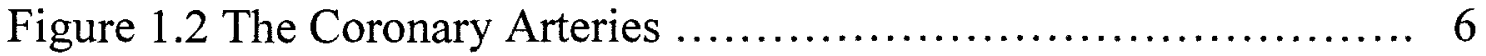

Figure 1.3 Abnormal Left Ventricular Wall Movements ................ 8

Figure 2.1 Cine - Angiograms Derived Contour Lines During Systole ... 20

Figure 2.2 Steps to Generate the 3-D Geometry ....................... 22

Figure 2.3 Alignment of the Geometry (Normal and Ischemic) .......... 23

Figure 2.4 Mesh Algorithm ..................................... 25

Figure 2.5 Mitral Valve's Movement During Systole .................. 27

Figure 2.6 (a) Simulated Ischemic Region (Small Ischemia on the Side). 28

Figure 2.6 (b) Simulated Ischemic Region (Large Ischemia on the Side). 29

Figure 2.6 (a) Simulated Ischemic Region (Large Ischemia at Back) .... 29

Figure 2.7 A Cross Section of LV with Three CERs ................... 32

Figure 3.1 Experimental Setup ................................. 36

Figure 3.2 DPIV System Components .............................. 38

Figure 3.3 Geometry Reconstruction ............................. 41

Figure $4.1 \mathrm{~b}$ Velocity and Vz Contour of Normal LV (NT1, NT2) ...... 55

Figure $4.1 \mathrm{~b}$ Velocity and Vz Contour of Normal LV (NT3, NT4) ...... 56

Figure 4.1c Velocity and Vz Contour of Normal LV (NT5) ............ 57

Figure 4.2a Velocity and Vz Contour of Ischemic LV (IT1, IT2) ...... 58

Figure 4.2b Velocity and Vz Contour of Ischemic LV (NT3, NT4) ..... 59

Figure 4.3a Velocity and $\mathrm{Vz}$ Contour of Simulated Ischemic LV

(Small Ischemia on the Side $F=0.5$ and $F=0.3$ ) ............6 60

Figure 4.3b Velocity and $\mathrm{Vz}$ Contour of Simulated Ischemic LV

(Small Ischemia on the Side $\mathrm{F}=0.1$ and $\mathrm{F}=0.0$ ) ............61

Figure 4.3c Velocity and $\mathrm{Vz}$ Contour of Simulated Ischemic LV

(Small Ischemia on the Side $\mathrm{F}=-0.1$ and $\mathrm{F}=-0.3$ ) ...........662

Figure 4.4a Velocity and $\mathrm{Vz}$ Contour of Simulated Ischemic LV

(Large Ischemia on the Side $\mathrm{F}=0.5$ and $\mathrm{F}=0.3$ ) ............63

Figure 4.4b Velocity and $\mathrm{Vz}$ Contour of Simulated Ischemic LV

(Large Ischemia on the Side $\mathrm{F}=0.1$ and $\mathrm{F}=0.0$ ) ...........64

Figure 4.4c Velocity and $\mathrm{Vz}$ Contour of Simulated Ischemic LV

(Large Ischemia on the Side $\mathrm{F}=-0.1$ and $\mathrm{F}=-0.3$ ) ..........66

Figure 4.5a Velocity and Vz Contour of Simulated Ischemic LV (Large Ischemia at Back $\mathrm{F}=0.5$ and $\mathrm{F}=0.3$ ) ..............66 
Figure 4.5b Velocity and $\mathrm{Vz}$ Contour of Simulated Ischemic LV

(Large Ischemia at Back $\mathrm{F}=0.1$ and $\mathrm{F}=0.0$ ) ................6 67

Figure 4.5c Velocity and Vz Contour of Simulated Ischemic LV

(Large Ischemia at Back $\mathrm{F}=-0.1$ and $\mathrm{F}=-0.3$ ) ..............68

Figure 4.6a Different Views of the CER inside the Normal LV (NT1) .. 69

Figure 4.6b Different Views of the CER inside the Normal LV (NT2) $\ldots 70$

Figure 4.6c Different Views of the CER inside the Normal LV (NT3) ... 71

Figure 4.6d Different Views of the CER inside the Normal LV (NT4) $\ldots 72$

Figure 4.6e Different Views of the CER inside the Normal LV (NT5) ... 73

Figure 4.7a Different Views of the CER inside the Ischemic LV (IT1) ...74

Figure 4.7b Different Views of the CER inside the Ischemic LV (IT2) $\ldots 75$

Figure 4.7c Different Views of the CER inside the Ischemic LV (IT3) $\ldots 76$

Figure 4.7d Different Views of the CER inside the Ischemic LV (IT4) $\ldots 77$

Figure 4.8a 3-D View of the CER inside LVs with Simulated Ischemic

Region (Small Ischemia on the Side) ...................... 78

Figure $4.8 \mathrm{~b}$ Front View of the CER inside LVs with Simulated Ischemic

Region (Small Ischemia on the Side) ..................... 79

Figure 4.8c Side View of the CER inside LVs with Simulated Ischemic

Region (Small Ischemia on the Side) .................... 80

Figure 4.9a 3-D View of the CER inside LVs with Simulated Ischemic

Region (Large Ischemia on the Side) .................... 81

Figure $4.9 \mathrm{~b}$ Front View of the CER inside LVs with Simulated Ischemic

Region (Large Ischemia on the Side) .................... 82

Figure 4.9c Side View of the CER inside LVs with Simulated Ischemic

Region (Large Ischemia on the Side) .................... 83

Figure 4.10a 3-D View of the CER inside LVs with Simulated Ischemic

Region (Large Ischemia at Back) ........................ 84

Figure 4.10b Front View of the CER inside LVs with Simulated Ischemic

Region (Large Ischemia at Back) .........................8 85

Figure 4.10c Side View of the CER inside LVs with Simulated Ischemic

Region (Large Ischemia at Back) ..........................86

Figure 4.11 CER Coefficient vs. Time Steps for Normal and Ischemic LV 88

Figure 4.12 CER Coefficient vs. Simulation Factor ..................... 89

Figure 4.13 CER coefficient vs. Simulation Factor based on Sphere Shape

Model

Figure 4.14 Velocity Vector Plots from the Numerical Simulation and 
Experimental Measurements for Normal LV ............. 91

Figure 4.15 Velocity Profile of Experimental and Numerical Results close to Outlet (Vz and Vy Component) ......................... 92

Figure 4.16 Velocity Profile of Experimental and Numerical Results close to Bottom (Vz and Vy Component) ......................... 93

Figure 4.17 Velocity Vector Plots from the Numerical Simulation and Experimental Measurements for Ischemic LV ............. 94

Figure 4.18 Velocity Profile of Experimental and Numerical Results close to Outlet ( $\mathrm{Vz}$ and $\mathrm{Vy}$ component) ........................ 95

Figure 4.19 Velocity Profile of Experimental and Numerical Results close to Bottom ( $\mathrm{Vz}$ and Vy component) ....................... 96

Figure 4.20 Comparison of the CER of Experimental and Numerical Results (Normal and Ischemic LV)

97 


\section{LIST OF TABLES}

Table 4.1 Time after Onset of Systole for Each Normal LV contour and corresponding Re for each time step ..................... 87

Table 4.2 Time after Onset of Systole for Each Ischemic LV contour and corresponding Re for each time step .......................

Table 4.3 CER Coefficients of Each Time Step of Normal and Ischemic LV ............................................... 88

Table 4.4 CER Coefficients if Simulated Ischemic LVs ............... 89 


\section{CHAPTER I}

\section{INTRODUCTION}

\section{I.1 Objective of the study}

Ischemic heart disease, which results from insufficient coronary artery blood flow, is one of the leading causes of mortality in developed countries. Other names for this condition include coronary heart disease and arteriosclerotic heart disease. Some deaths occur suddenly as a result of an acute coronary occlusion or of fibrillation of the heart, whereas others occur slowly over a period of weeks to years as a result of progressive weakening of the heart pumping process.

The resulting abnormal function of the heart has been assessed for diagnostic and prognostic purposes predominately and most successfully by the ejection fraction (Rahimtoola, 1982), a global measurement relating stroke volume to available blood volume in the left ventricle. However, coronary artery disease produces localized areas of abnormal wall movement which may vary with time within the cardiac cycle, and compensatory actions in the unaffected regions of the myocardium can 
result in inadequate characterization of the state of disease using parameters based on global measures alone.

The advent of noninvasive imaging technology such as echocardiography has led to a rapid growth of studies related to left ventricular function. Parameters, which based on the image, had been shown to have the ability to estimate the ventricular performance (Nobuyuki et al. 1992). Regional wall motion analysis has also begun to be used as an adjunct tool in function assessment. However these techniques only provide information about small regions without supplying a measure of the heart's overall function.

To describe the overall left ventricular function, a quantitative index of global left ventricular function based on regional wall motion has to be universally agreed upon (Clayton et al., 1984; Owen et al., 1991). An accurate method to quantify the degree of ischemia to aid in choosing approriate treatment to prevent furthers myocardial damage also needs to be developed.

The objectives of this study are the development of a threedimensional numerical model that has realistic left ventricular geometry; Modification of Central Ejection Region (CER) as well as its quantitative 
version CER coefficient, which is a potential diagnostic index of left ventricular function evaluation and assessment, from the two-dimensional flow to one suitable for three-dimensional flow; Verification of the above numerical model through in vitro experimental measurements.

\subsection{Physiology of human heart}

The human heart is a hollow muscular, conical shaped organ located obliquely between the lungs, and enclosed in the cavity of the pericardium. The base is directed upward, backward and to the right. The apex is directed downward, forward and to the left. In adults, the heart measures about five inches in length, three inches and a half in breadth in its broadest part. It weights from ten to twelve ounces in males and eight to ten ounces in females [23].

The heart, by virtue of the contractile activity of its muscular walls, propels blood throughout the body so as to deliver oxygen and nutrients to and removes waste from each of the organs. It also provides for the transport of hormones and other regulatory substances between various regions of the body. 
The human heart (Figure 1.1) consists of two separate pumps; each composed of an atrium and a ventricle. The right side is responsible for supplying blood to the pulmonary circulation while the left side is responsible for supplying blood to the systemic circulation.

The atria are collecting chambers, and the ventricles are pumping chambers. The right ventricle receives blood from the right atrium and pumps it into the pulmonary circulation; the left ventricle receives blood from the left atrium and pumps it into the systemic circulation. Between the cavities of the atria and ventricles lie the atrioventricular valves: on the right the tricuspid valve and on the left the mitral valve. These valves prevent the back flow of the blood from the ventricles to the atria when the ventricles contract.

The aortic valve and pulmonary valve, which situate at the outflow of the ventricles, prevent back flow of the blood from the aorta and pulmonary artery into the ventricles when they relax.

Both atria are thin-walled muscular chambers. The thinness of their walls reflects the low pressures normally developed in the atrial cavities. The ventricles, on the other hand, have thick muscular walls, especially the left ventricle, which has approximately three times the mass and twice the 
thickness of the right ventricle. The left ventricle is longer and more conical in shape than the right ventricle. It resembles an elongated cone with inflow and outflow tracks adjacent to each other. By contrast, the right ventricle has more of a crescent shape with separated tracks.

The interior surfaces of the heart are lined by a thin and smooth membrane called the endocardium. The outer surfaces are covered by a protective connective tissue called the epicardium. The region between the epicardium and the endocardium is referred to as the ventricular myocardium, which is formed by a series of overlapping muscle bundles spiraling from the fibrous base to the apex.

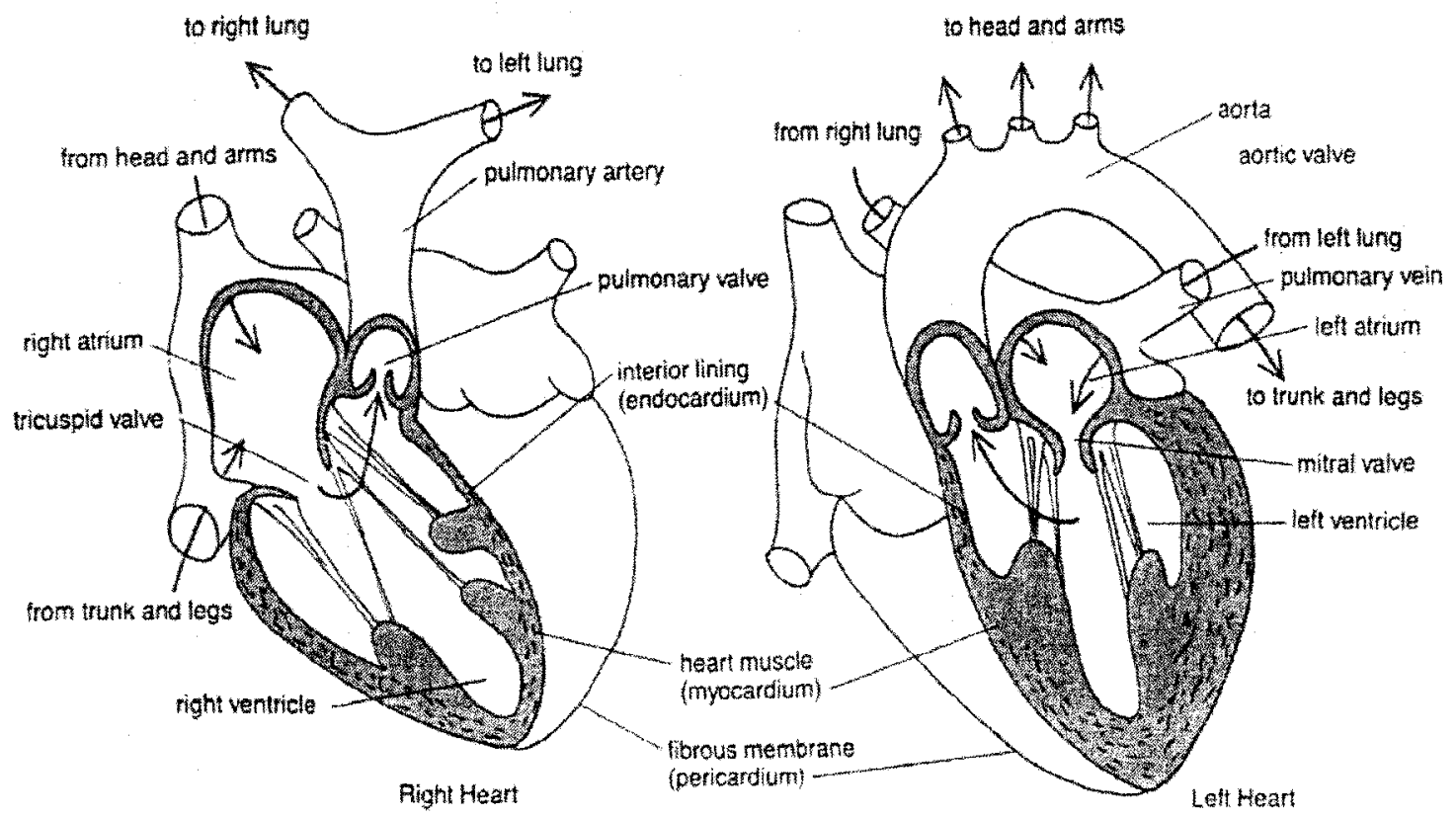

Figure 1.1 The anatomy of the human heart [5] 


\section{I.2.2 Ischemic heart disease}

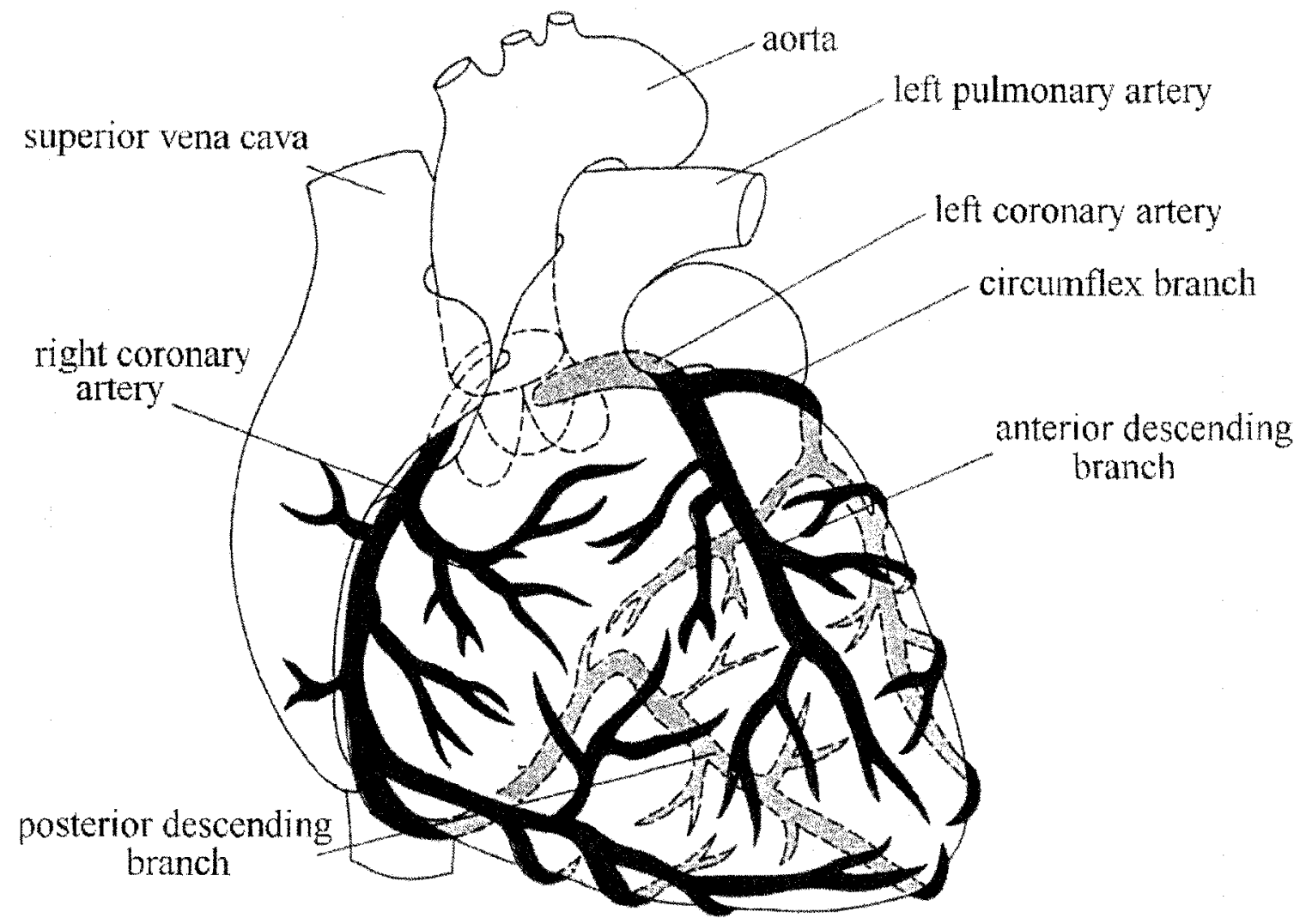

Figure 1.2 The Coronary Arteries [23]

Blood is transported to the heart muscle by the left and right coronary arteries, which arise at the root of the aorta behind the right and left cusps of the aortic valve, and their many branches, reaching the myocardium by way of small arteries. Approximately $5 \%$ of the total blood flow from the left ventricle goes through the coronary circulation. The oxygen needs of the heart muscle are only minimally met by this amount of blood flow. Any 
constrictions of the coronary arteries and their branches may lead to damage of the heart muscle by restriction of its blood supply. This symptom is referred to as ischemic heart disease.

By far the most frequent cause of diminished coronary artery blood flow is atherosclerosis. This may be due to abnormalities of lipid or carbohydrate metabolism, or may be the result of organizing thrombosis. In certain persons, large quantities of cholesterol gradually deposited at many points in the arteries. Later on, these deposits become invaded by fibrous tissue, and they also frequently become calcified. The net result is the developments of atherosclerotic plaques that protrude into the vessel and either completely block or partially block blood flow. A very common site for development of atherosclerotic plaques is the first few centimeters of the coronary arteries. When this situation becomes severe and beyond any compensatory mechanism, acute coronary occlusion occurs leading to ischemia and infarction of the affected area. The regional heart muscle infarction may cause a decrease in local contractility so as to reduce the heart's overall pumping function.

Three kinds of abnormal wall motion have been observed on ischemic heart: hypokinesis, in which the infarct area has a reduced 
contraction; akinesis when the infarct area is absence of contraction; and dyskinesis for which the infarct area produces paradoxical motion. Figure 1.3 shows these different wall movements, where arrows indicate the direction of the wall movement.

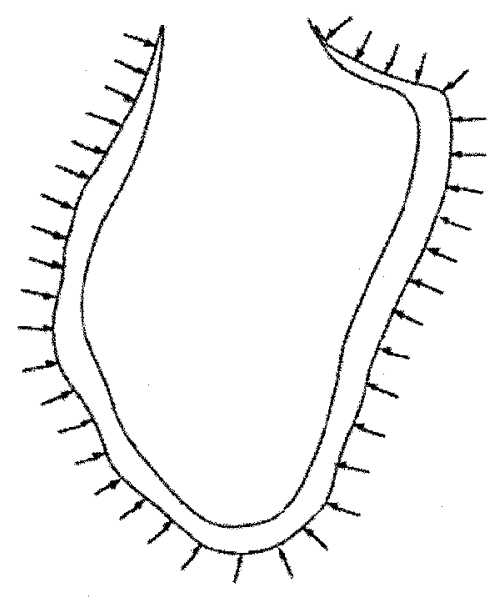

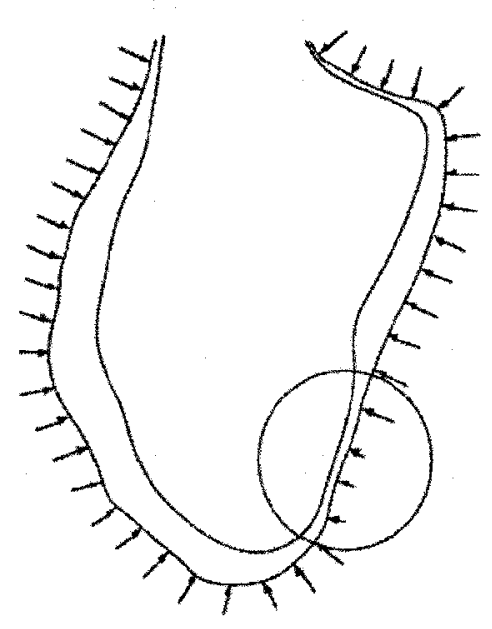

Hypokinesis

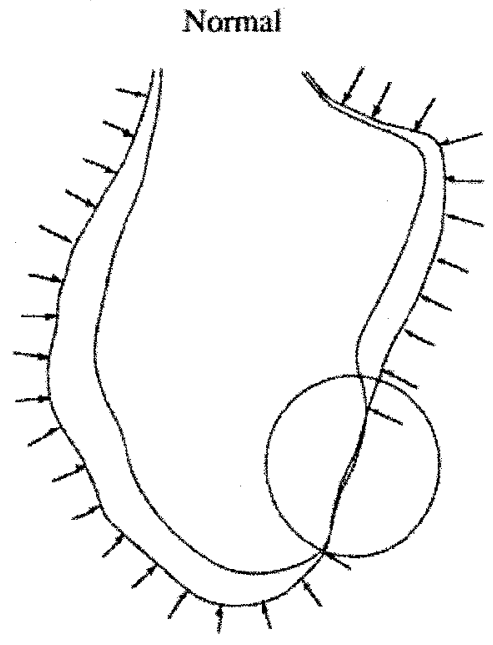

Akinesis

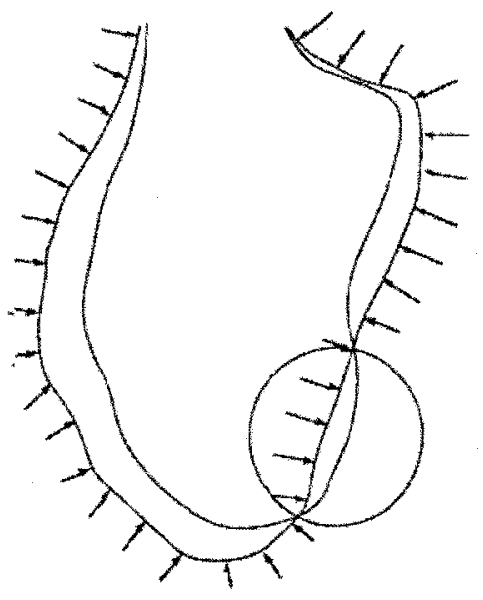

Dyskinesis

Figure 1.3 Abnormal left ventricular wall movements [4] 


\section{I.3 Previous Study}

For the past two decades, computational fluid dynamics has been a useful research tool in the study of cardiovascular fluid mechanics. The ultimate goal is to develop a computational scheme in which realistic threedimensional flow simulations can be achieved (Wendt, 1992).

The earliest work that solving biomechanics problems using computational fluid dynamics found in the literature was back in 1972, Peskin numerically solved the Navier-Stokes equations in the presence of moving immersed boundaries that moved by the local velocity of the fluid and exerting forces. In 1977 Peskin improved the method by including the muscular heart wall.

By the late 1980 's, it was feasible to simulate three-dimensional timedependent flow. Three-dimensional equations of a viscous incompressible fluid that contains an immersed system of elastic fibers and contractile fibers were solved (Peskin et al. 1988, McQueen et al. 1988). Recently, Yoganathan et al. (1994) solved the three-dimensional Navier-Stokes equations for time-dependent flow in a compliant thin-walled, anatomically correct left ventricle during early systole. He also used the magnetic resonance imaging (MRI) technique to measure the three-dimensional 
velocity in a MRI slice through a human subject, making MRI a means of obtaining the intravenricular flow field (Walker et al. 1996).

All the studies (Georgiadis et al. 1992, Hampton et al. 1992, Redaelli et al. 1996, Taylor et al. 1994 and 1995) which focused on the fluid dynamics inside the left ventricular chamber modeled blood as the Newtonian flow with the constant properties. The wall mechanics was not included.

Due to the irregular geometry, complicated movement and the lack of three-dimensional data, most studies (Georgiadis et al. 1992, Gonzalez et al. 1996, Redaelli et al. 1996, Taylor et al. 1994) utilized simple geometry models to study the left ventricular ejection. The movement of the left ventricular wall was simulated by the inflow across the wall.

Taylor et al. used three-dimensional radius-varying spherical models, and the boundary condition was calculated from the volume reduction. Boundary nodes were assumed to move towards the outlet center (Taylor et al., 1994). A year later, Taylor et al. (1995) built a realistic threedimensional left ventricular model to study the ejection fluid dynamics indicating that there is a definite difference in the geometric shapes and flow patterns in normal and infarct hearts. 
Georgiadis et al. (1991) introduced a two-dimensional ellipsoidal cavity by using different chamber eccentricities. Boundary condition was assumed to be uniform and perpendicular to the wall. This model was extended to three-dimension (Georgiadis et al., 1993). Schoephoerster et al. (1993) built a two-dimensional real-shape model from a series of digitized echocardiograms. The boundary nodes were assumed to move towards the closest point on the geometry of next time step.

Gonzalez et al. (1995) solved equations for a three-dimensional spherical geometry with the same assumption of boundary condition.

Redaelli et al. (1996) utilized a simple contracted moving boundary condition on an axis-symmetric finite element model.

Quantitative experimental measurements of the left ventricle flow are very limited. Schoephoerster et al. (1991) measured velocity of different positions past-mitral valve in a rigid body left ventricle with LDA.

With the development of different heart imaging technologies, most of the work in this area has been directed towards detailing the actual movement of the left ventricular wall (Sennan et al., 1986), or towards determining the rheological and mechanical properties of the myocardium (McPherson et al., 1987) from the image information. Very little attention 
had been given towards the analysis of regional flow patterns within the left ventricular chamber, which is undoubtedly influenced by the time dependent regional movement of the left ventricular wall.

Schoephoerster et al. (1993) introduced a two-dimensional model of left ventricular flow dynamics. Based on that model, Schoephoerster et al. (1994) further studied the relation between left ventricular function and the flow patterns based on left ventricular wall motion. In this study, an index which has the potential to describe left ventricular global function based on regional and temporal variations in left ventricular wall motion was developed: the Central Ejection Region (CER), as well as its related quantitative index, the CER coefficient.

CER is the region of flow domain, which is aligned for ejection. It is defined as the flow domain in which the velocity direction is within five degrees of the left ventricular long axis. The data from this study shows that the CER is sensitive to regional decreased wall movement and the severity of the ischemia. Under normal wall motion conditions, the CER mainly follows the symmetric line of the left ventricle. However, in ischemic cases, the CER becomes thinner, shorter, and even breaks into disconnected small pieces for some very severe cases. The end of the CER tends to shift 
towards the ischemic region. The average CER coefficient for the ischemic left ventricle was lower than that for normal left ventricle; it also decreased with the increase of the severity of simulated ischemic cases.

Gonzalez (1995) extended Schoephoerster's work to a threedimensional sphere-shaped model with both normal and simulated ischemic cases. The results further indicated that CER is a valuable left ventricular pumping efficiency index. CER coefficient quantitatively shows that the flow patterns are rather sensitive to moderated degrees of hypokinesis. Also Gonzalez emphasize the importance of building the three-dimensional model using the real left ventricular wall motion as input to the computer model.

The present study is a further step of the work done by Schoephoerster and Gonzalez. Yet, it is still a preliminary step of the complete understanding of left ventricular flow dynamics. Two goals were accomplished: 1) development of a three dimensional more realistic numerical model as well as the CER; and, 2) verification of the numerical method by experimental measurements using digital particle image velocimetry (DPIV). 
Our ultimate aim is to develop and use the diagnostic index presented as an improved clinical tool to complement the capabilities of newer generation MRI machines which will be able to directly measure velocity patterns.

\section{I.4 Problem Statement}

When its wall expending and contracting periodically during each cardiac cycle, the human left ventricle is a three-dimensional irregular chamber, which continuously changes its geometry. The accurate numerical or experimental simulation of the flow within this chamber would be extremely complex, which would require difficult manufacturing technique, large amounts of computer resources as well as a complicated numerical method, therefore, is beyond the scope of this study.

In the current study, a simplified model was utilized to compute the velocity field based on the wall motion. The results were used to primarily study how normal and varying abnormal wall contractions affect the cavity flow dynamics. Since the mitral valves remain closed, while the aortic valves remain open during systole, the geometry of the control volume was modeled as a contracting chamber with a single outlet. 
The Navier-Stokes equations were used to describe the flow inside the left ventricle. A general-purpose flow dynamics software package FIDAP was used to solve the digitized Navier-Stokes equations.

An index, which can be used to describe the function of the left ventricle, was concluded from the numerical results: central ejection region (CER). It represents the region in which the blood inside the left ventricle is aligned to eject. The CER appears to be a useful tool to visualize changing flow with changes in wall motion resulting from changes in the severity of the simulated ischemia. 


\section{CHAPTER II}

\section{MATHEMATICAL EQUATIONS \&}

\section{NUMERICAL METHODOLOGY}

\section{II.1 Problem Definition}

As mentioned in the previous chapter, the model of the current study is based on a known control volume, which is a contracting chamber with a single outlet. The velocity field was computed based on the wall motion. The results were used to primarily study how various wall contractions affect the chamber flow dynamics.

The model was built on the following assumptions: The inside flow was steady, laminar and incompressible. The wall was impermeable, nonslip with a prescribed contraction. The wall mechanics were not included in this model. The actual boundary conditions were simulated by the inflow across the left ventricular wall. At the outlet, velocity was uniform. Since the average shear rate inside the left ventricle was expected to exceed $50^{-1}$ sec, the inside flow was considered to behave as a Newtonian fluid. The temperature and the physical properties of blood were assumed to remaine unchanged. Gravitational effects were ignored. 


\section{II.2 Mathematical Governing Equations}

The governing equations for the problem were the continuity equation and the Navier-Stokes equations for incompressible, steady flow with constant properties and no gravitational iterms:

$$
\begin{aligned}
& \frac{\partial U i}{\partial x i}=0 \\
& \frac{\partial U i}{\partial t}+U j \frac{\partial U i}{\partial x j}=-\frac{1}{\rho} \frac{\partial P}{\partial x i}+\mu \frac{\partial}{\partial x j}\left(\frac{\partial U i}{\partial x j}\right)
\end{aligned}
$$

where

$i=1,2,3$

$U_{i}=$ velocity component in the ith direction

$x_{i}=$ ith direction

$P=$ pressure

$\rho=1.1 \mathrm{~g} / \mathrm{cm}^{3}$, fluid density

$\mu=3.5$ poise, fluid viscosity

Equation (2.1) and (2.2) represent a system of four equations with four unknowns: $U_{i}$ and $P$. With the proper boundary and initial conditions, this system of equations has a unique solution. 


\section{II.3.1 Numerical Method}

Finite element method (FEM), which has found increased use and wider acceptance for the solution of the equations governing viscous fluid flows in recent years, was adopted to solve the governing equations. A general-purpose code FIDAP (Fluid Dynamics International, Evanston, IL 60201) was utilized for the calculation.

The full Navier-Stokes equations describing the flow domain were solved using Galerkin's weighted residual approach in conjunction with finite element approximation. To reduce disk storage requirements, a segregated algorithm was used to solve the nonlinear system of matrix equations arising from the FEM discretization of the flow equations.

All the computations were carried out on a Silicon Graphics Power Challenge Server.

\section{II.3.2 Formulations of The Discrete Problem}

The objective of FEM is to reduce the continuum problem to a discrete problem described by a system of algebraic equations. It begins with the division of the continuos flow domain into a number of simply shaped elements. Within each element, variables $u_{i}$ and $p$ were interpolated 
by functions of compatible order, in terms of values to be determined at nodes. The discrete analogue of equation (2.1) and (2.2) for an individual element can be expressed by the following matrix equations:

$$
\begin{aligned}
& M \dot{U}+A(U) U-C P=0 \\
& C^{T} U=0 \\
& \text { where } U=\left(U_{1} U_{2} U_{3}\right)^{T}
\end{aligned}
$$

where A represents the convection of momentum. $M$ represents the mass term in the field equations.

Usually the FEM is not applied directly to the full system of governing equations but rather to a perturbed system of equation in which the continuity requirement is weakened and replaced by:

$$
u_{\mathrm{i}, \mathrm{j}}=-\varepsilon p
$$

Where $\varepsilon$ is a small number, typically between $10^{-5}$ and $10^{-9}$. This approach, referred to as a penalty function approach, has the great advantage of eliminating the dependent variable $p^{\varepsilon}$, which is then recovered by post-processing from the velocity field by,

$$
p^{\varepsilon}=-\frac{1}{\varepsilon}{ }_{u_{i, j}}^{\varepsilon}
$$


When the penalty formulation is employed, equation (2.4) was replaced by:

$$
C^{T} U=-\varepsilon M_{p} P
$$

Further, $P$ can be eliminated from equation (2.3):

$$
M \dot{U}+A(U) U-\frac{1}{\varepsilon} C_{M_{p}}{ }^{-1} C^{T} U=0
$$

\section{II.4.1 Geometry Generation}

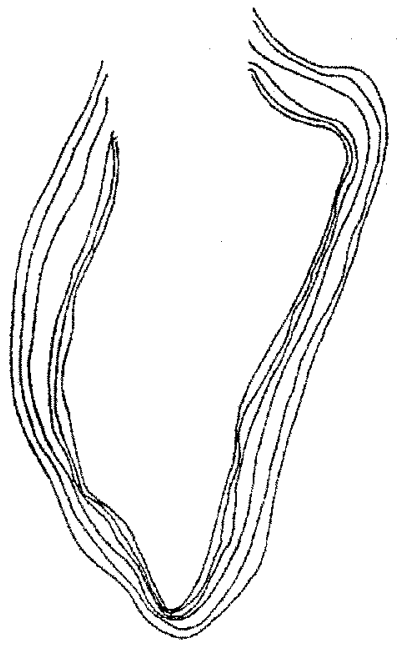

(a)

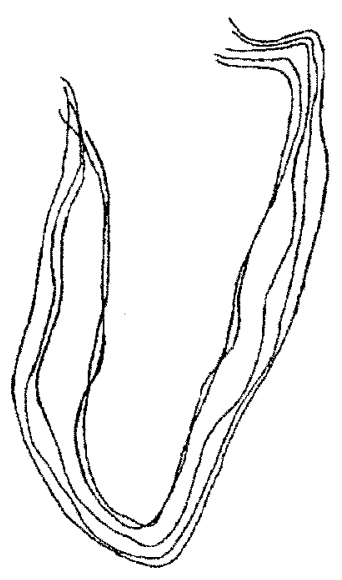

(b)

Figure 2.1 Cine - Angiograms derived contour lines during systole
(a) Normal left ventricle
(b) Ischemic left ventricle

The three-dimensional geometries of the left ventricle used in the study were reconstructed from the digitized two-dimensional cineangiograms of real patients (Figure2.1). Based on 30 frames per second rate, six curves which represented the contour of left ventricle along the 
long axis during systole were obtained from a normal left ventricle and five were obtained from a left ventricle with known ischemia. Both left ventricles have similar ejection fraction, and no apparent abnormal wall motion from end-diastole to end-systole.

Three dimensional geometry generation starts with all the curves placed on the $\mathrm{Y}-\mathrm{Z}$ plane, the long axes aligned with $\mathrm{Z}$-axis, and the simple steps:

1. Each curve was broken into two parts at the apex, which is the farthest point from the outlet center. Then each half was divided into 200 equal length curve segments (Figure 2.2a).

2. The corresponding separation points on both halves were connected by a line segment (Figure $2.2 \mathrm{~b}$ ).

3. Each line segment was translated to a new coordinate system in which the line was aligned with the $\mathrm{Y}$-axis and perpendicular to the Z-axis. Then the line was rotated 180 degrees according to the Zaxis and its center. The trace of its two ends formed a closed circle (Figure 2.2c). 
4. Two hundred equally distributed points on each circle were digitized and reverse translated back to its original coordinate system (Figure 2.2d).

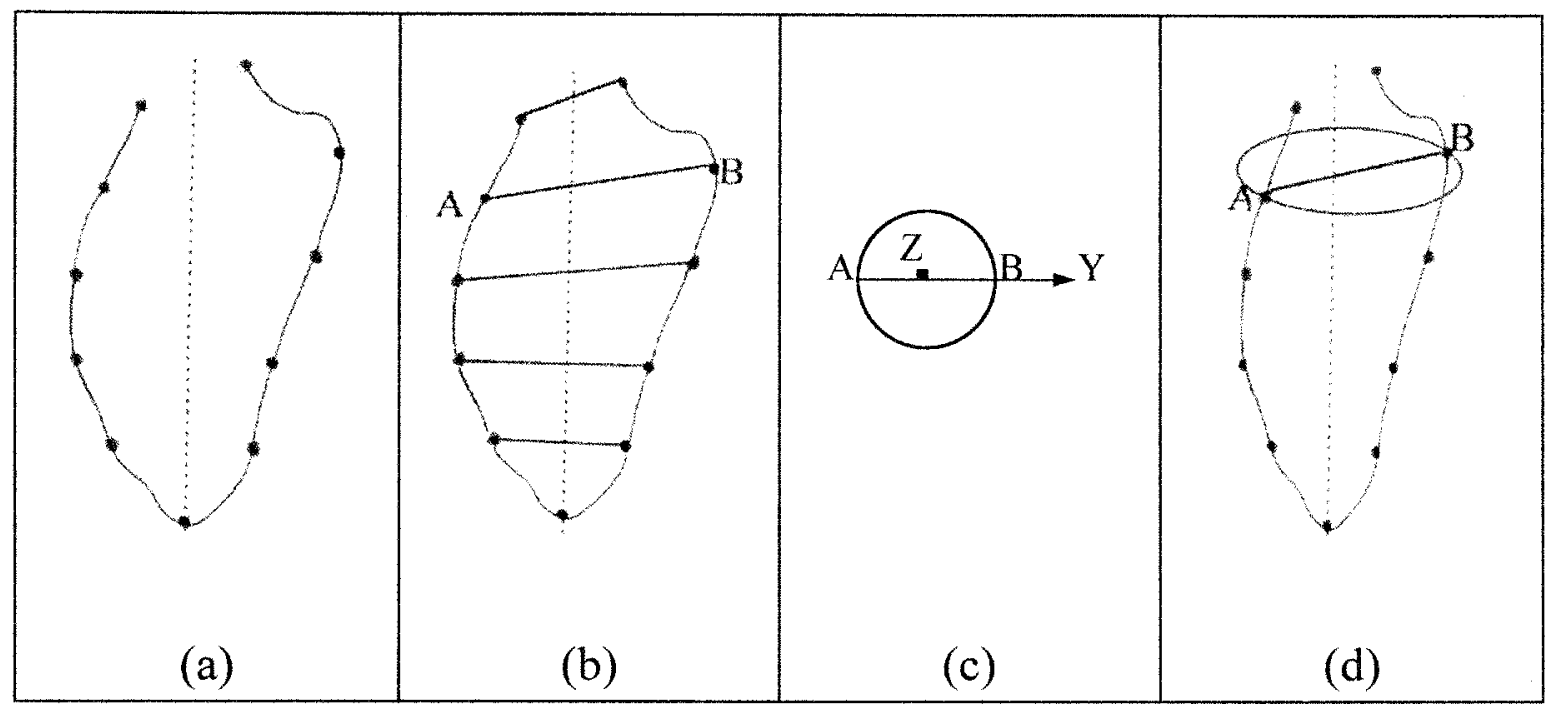

Figure 2.2 Steps to generate the 3D geometry

Thus each three-dimensional geometry is consisted of two hundred tilted circles and one apex point. Two hundred points were digitized on each circle, for a total of forty thousand and one points on one geometry.

Step 1 was accomplished utilizing a function inside FIDAP to equally divide the curves and output the coordinates of each separate point. The rest was done by a computer code GEO-GEN.c written in C language. The main mathematical theory used was the coordinate system transformation. The source code is presented in Appendix A. 


\section{II.4.2 Geometry data modification}
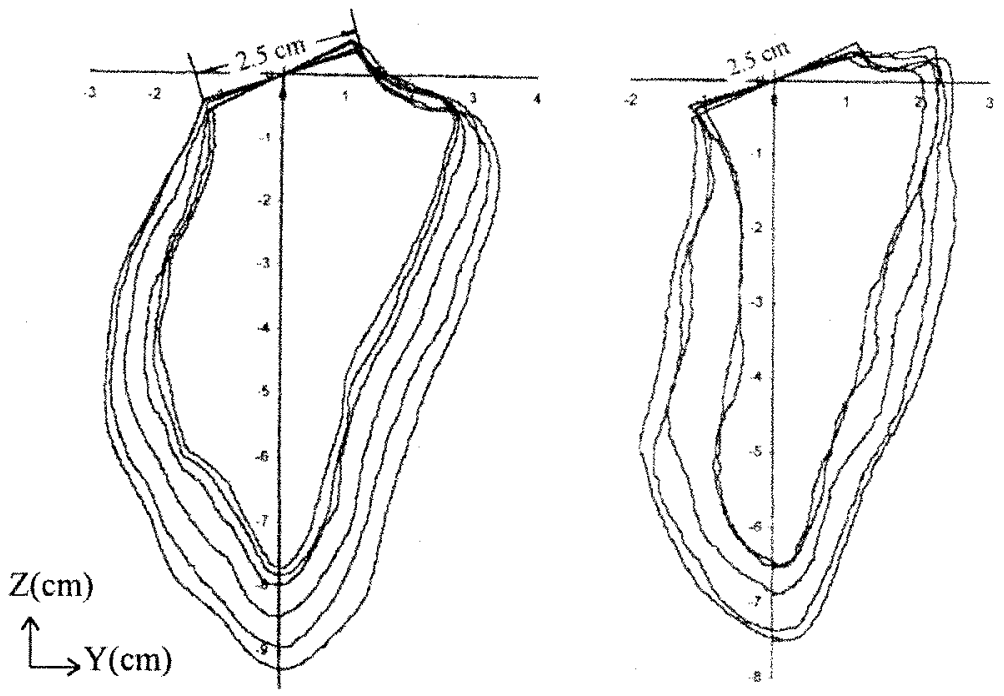

Figure 2.3 Alignment of the geometry (Normal and Ischemic)

As mentioned before, the original two-dimensional geometries were obtained from real patients. Figure 2.1 has captured the actual motion of the left ventricle, which includes rigid body movement and the contractile deformation. These curves were digitized based on a static reference point, with the measurement unit pixels.

To avoid additional complications that would result from taking into account inertial effects due to the rigid body, some modifications were necessary.

Figure 2.1 shows the geometry after the modification: all the centers of the outlet were moved to the origin, with every long axis aligned with the 
Z-axis (Figure 2.2). The outlet diameter of the first geometry was translated to $2.5 \mathrm{~cm}$, which is the average size of the human aortic valve.

\section{II.4.3 Mesh Algorithm}

FIDAP requires that the computational domain to be defined by mesh solids to apply the meshing process. The contours of the mesh solid follow the contours of the flow domain. The only map meshing method, which is available for three-dimensional domains, is a regular "checkerboard" shape meshing.

To keep the element aspect ratio close to 1 , the entire flow domain was decomposed into fifty-two sub-domains. Each was defined by one mesh solid. Adjacent domains were connected by the mutual mesh surface. All the mesh solids were meshed into eight-node isoparametric brick elements (Figure 2.3). There were a total 22560 elements and 21429 nodes generated for each geometry. This is the maximum node number allowed by the memory size and disk space of our computer to perform the correct computation. 


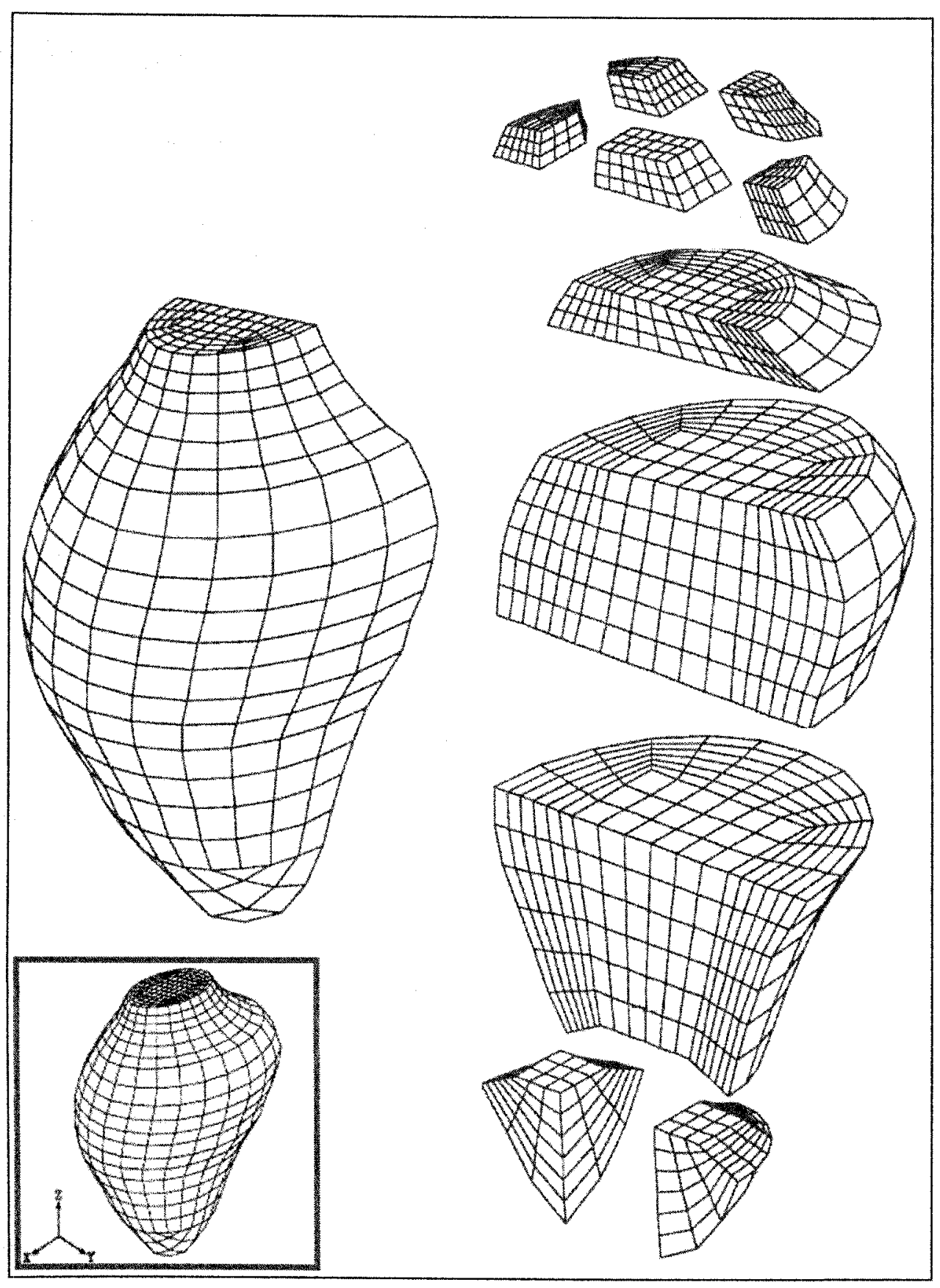

Figure 2.4 Mesh algorithm 


\section{II.5.1 Boundary conditions}

It was assumed that during the time period between two adjacent left ventricular geometries, the left ventricular wall moved so insignificantly that the wall movement could be regarded equivalent to the flow across the wall. Thus the prescribed-wall-motion boundary was substituted by the flow across the wall. The time period between two geometries varies from $67 \mathrm{~ms}$ to $134 \mathrm{~ms}$.

The boundary conditions of each node were computed from two adjacent left ventricular geometries. Each node on the first geometry wall was assumed to move towards the position, which was occupied by the closest point on the next geometry at a constant velocity. The coordinates of each node on the wall were output from FIDAP. The displacement between each node and its closest point on the next geometry were calculated, then divided by time period between these two geometries to get velocity boundary conditions.

Although mitral valves remain closed during systole, they do not contract like the muscular wall. On the other hand, it has paradoxical movement under the pressure, which is built up inside the left ventricle (Figure 2.4). This paradoxical movement caused a vortex near the outlet 

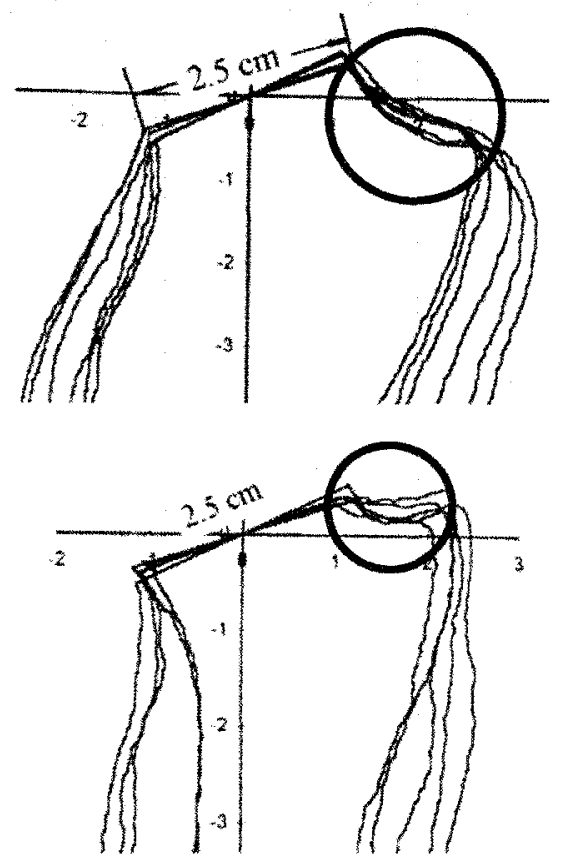

Figure 2.5 Mitral valve's movement during systole

affecting the convergence of the entire

flow domain. Since we are only interested in the main ejection region, all the boundary velocities of mitral area were set to zero to eliminate the extra computation caused by this effect.

The displacement and velocity boundary conditions were calculated by program BCondition.C, which is presented in Appendix B.

The boundary velocity at the outlet was assumed to be uniform and perpendicular to the outlet surface. The magnitude was calculated from the mass conservation inside the control volume.

\section{II.5.2 Simulated ischemic boundary conditions}

A better understanding of how ischemia affects the left ventricular flow pattern development may be obtained by controlling the severity or the location of the ischemic region. For this purpose, a simulated ischemic region was imposed on the normal left ventricle wall to simulate ischemia. 
Since the normal left ventricle has the most symmetric contraction during the $\mathrm{T} 1$ time step, all the simulations were applied on the geometry of this time step.

Figure 2.5a-c show three simulated ischemic left ventricular models with the ischemia of different sizes or positions we used in the study: small ischemia on the side, large ischemia on the side and large ischemia at back.

The gray area indicates the simulated ischemic region.

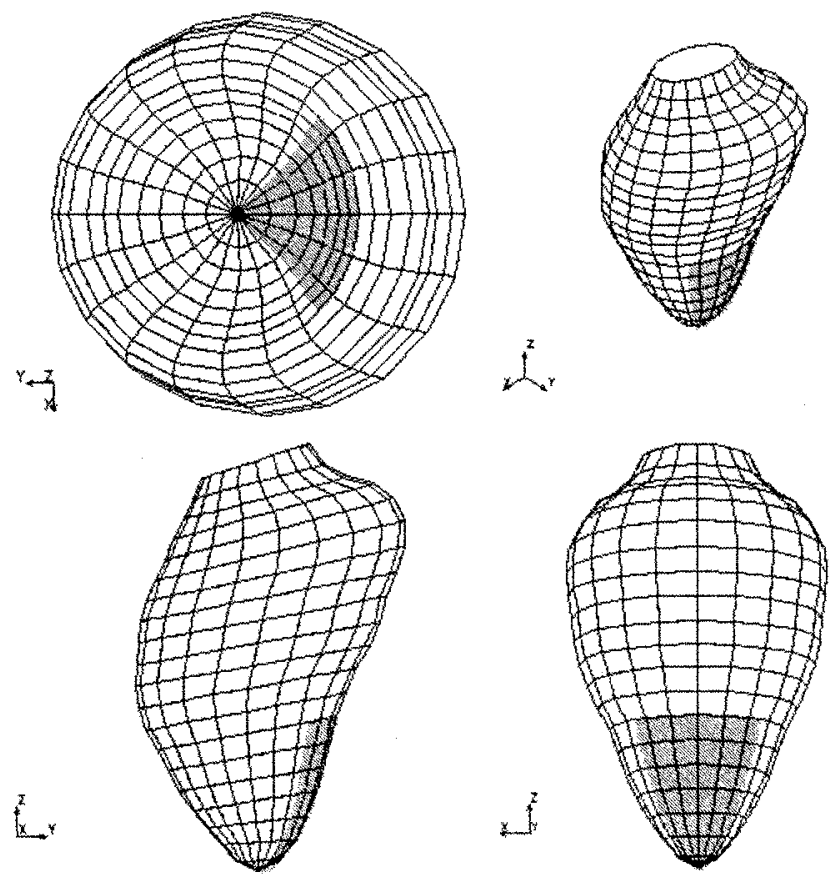

(a)

Figure 2.6 Simulated ischemic region

(a)small ischemia on the side (b)large ischemia on the side (c)large ischemia at back 

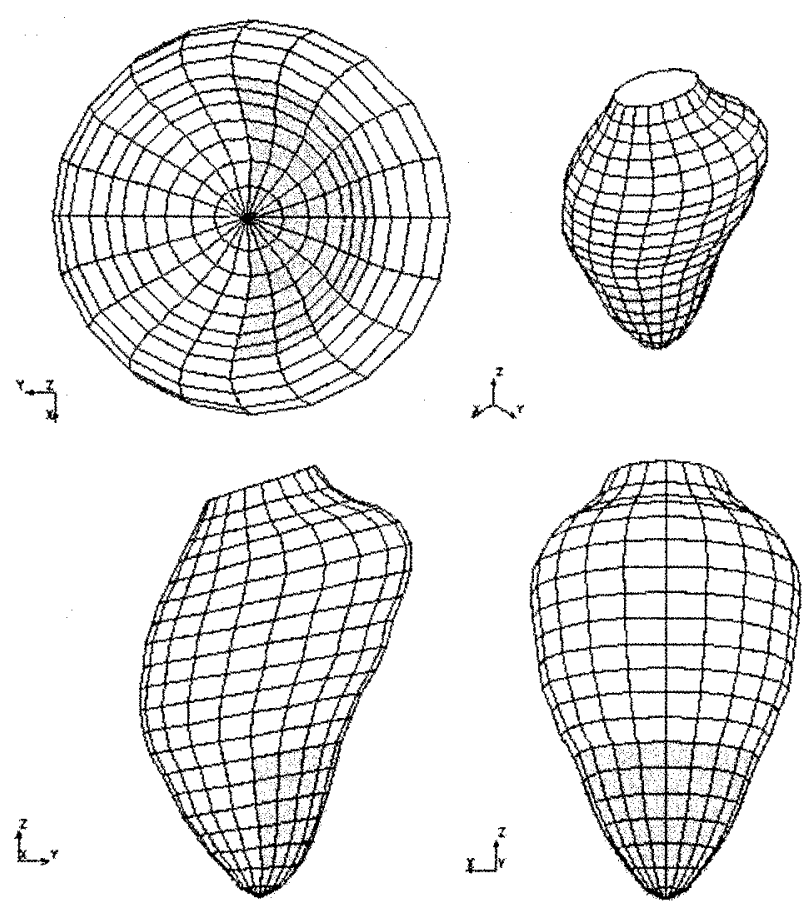

(b)
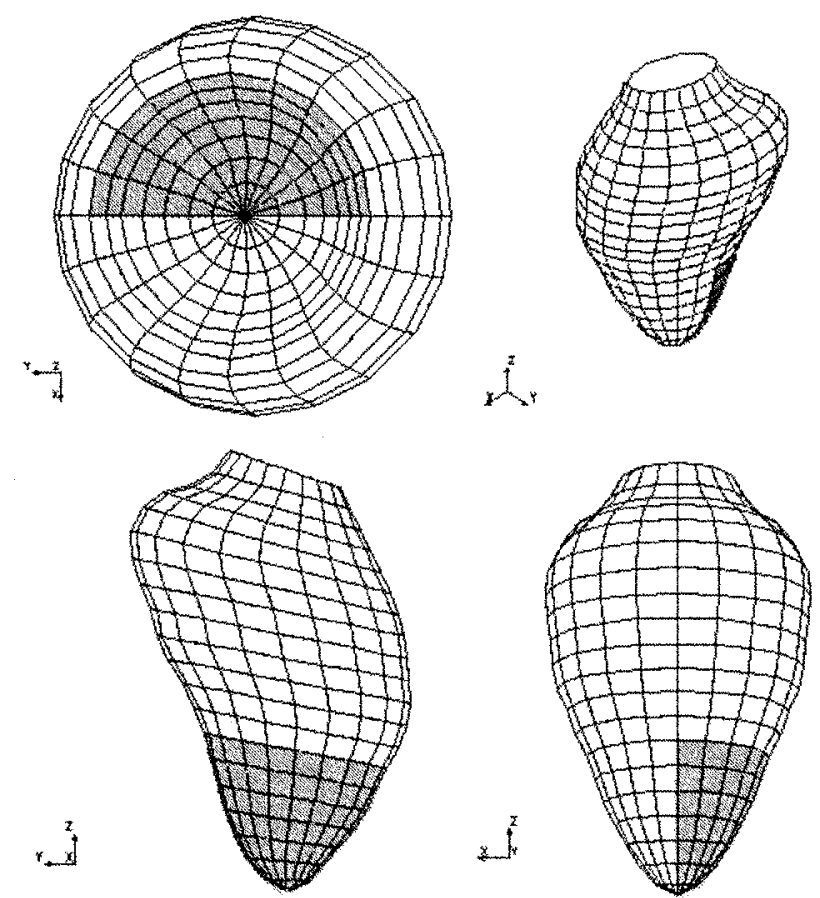

(c)

Figure 2.6 Simulated ischemic region

(a)small ischemia on the side (b)large ischemia on the side (c)large ischemia at back 
As mentioned before, each geometry is consists of 201 layers of digitized points including the apex. The simulated ischemia was on the bottom 67 layers, which is approximately one third of the total height. The small region extended around 45 degrees with respect to $\mathrm{Y}$-axis. The large regions extended around 90 degrees with respect to the $\mathrm{Y}$-axis or $\mathrm{X}$-axis.

A simulation factor $\mathrm{F}$ was used to control the severity of the ischemia. It represents the percentage of the normal velocity boundary conditions and was multiplied to the velocity normal boundary condition of all the nodes located in the ischemic region. When $\mathrm{F}$ is between 0 and 1 , the normal velocity boundary conditions were reduced to a certain percentage, and hypokinesis was simulated. $\mathrm{F}$ of 0 reduced all the velocity boundary conditions to 0 and simulated akynises. When $F$ is less than 0 , the magnitude of the velocity boundary condition was reduced and the direction of it was reversed, thus dyskinesis was simulated.

For each ischemic region, three hypokinesis, two dyskinesis and askynesis were simulated with $F$ values of $0.5,0.3,0.1,0,-0.1$ and -0.3 .

The computation of simulated boundary conditions was performed by program BCondition.c. 


\section{II.6.1 Central Ejection Region(CER) and CER coefficient}

To extend the CER defined for the two-dimensional numerical model (Schoephoerster 1993), the CER as well as its quantitative version the CER coefficient were developed for the three-dimensional realistically shaped model.

CER is defined as the region in which the velocity direction is within 5 degrees with the left ventricular long axis. The long axis is the line segment connecting the center of the outlet and the apex. Physically, CER is the flow domain in which the flow is aligned to eject. So the larger the CER, the more efficient the left ventricle is.

It is a common assumption that an ideal ventricle should contract symmetrical to the centerline, especially the left ventricle that has a pearshape chamber. So we expected that the CER of a healthier left ventricle would have a better alignment with the centerline than the one of an ischemic left ventricle.

The CER coefficient is a number based on the CER which can quantitatively described the left ventricular ejection. It is defined as:

$\mathrm{C}_{C E R}=\frac{\sum_{1}^{N}\left(1-\frac{1}{M} \sum_{1}^{M} \frac{d_{C E R}}{d_{B L}}\right)}{N}$ 


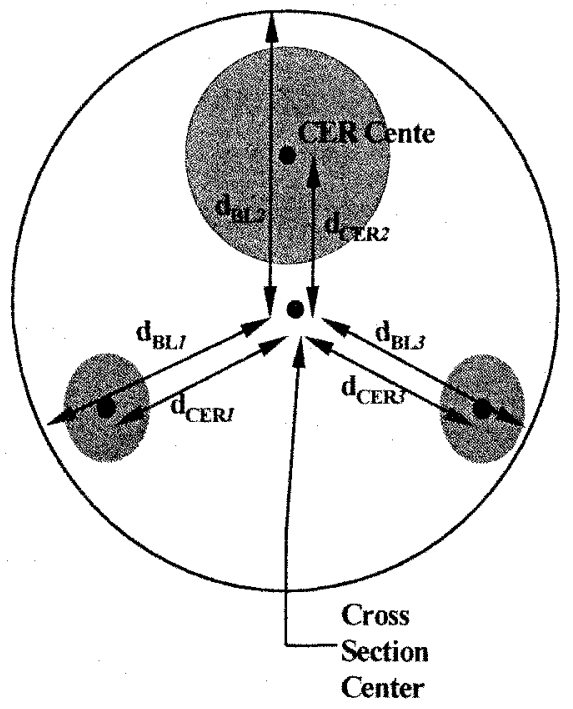

Figure 2.7 A Cross Section Of LV with Three CERs distributed horizontal cross sections from the outlet to apex. $d_{B L}$ is the distance from the left ventricular wall to the geometric center of a particular cross section, $d_{C E R}$ is the distance from the center of the same cross section to the center of the CER cross section. $M$ is the number of the CER regions on the same cross section. If a particular cross section had no CER, $d_{C E R}$ was set equal to $d_{B L}$.

From equation (2.9) we can see that CER coefficient is between 0 and 1. A value of one indicates that the CER center is aligned with the cross section center on every cross section. That means that the CER follows the left ventricular centerline perfectly, indicating an ideal contraction. Whereas a value of zero means that all the flow has been pushed to one side of the left ventricle instead of the outlet indicating a poor contraction. 


\section{II.6.2 Data Processing}

The calculation of CER coefficient was based on the velocity information of the positions on $\mathrm{N}$ horizontal planes within the flow domain. $\mathrm{N}$ was 50 in the present study. Unlike the Finite Analytic Method grid, which was used by Silva (1991) and Gonzalez (1994), the FEM mesh doesn't naturally meet this condition. So the entire velocity field was interpolated into a new mesh having 50 equally distributed horizontal planes from the lowest point of the outlet to apex, all the nodes on each plane evenly distributed in the $\mathrm{X}$ and $\mathrm{Y}$ direction.

It is necessary to numerically find the geometric center for each horizontal cross section and the geometric center of CER on each cross section to obtain the $d_{B L}$ and $d_{C E R}$. The curve, which is made by connecting the center of each layer of the geometry, is considered to be the centerline of the left ventricular cavity. The gravity center of the CER was considered the CER center. Its coordinates were determined by:

$$
\begin{gathered}
X_{C E R}=\frac{\sum_{N} X_{i} A_{i}}{\sum_{N} A_{i}} \\
Y_{C E R}=\frac{\sum_{N} Y_{i} A_{i}}{\sum_{N} A_{i}}
\end{gathered}
$$


where $X_{i}$ and $Y_{i}$ are the coordinates of the nodes within the CER, and $A_{i}$ is the area summation of the elements around the node.

To obtain $d_{B L}$, the local radius, which went through the CER center, was used. The angle between the X-axis and the line segment connecting the cross section center and CER center and the angles between the X-axis and the every radius on the cross section were compared. The radius whose angle with the $\mathrm{X}$-axis is the closest to the angle between the $\mathrm{X}$-axis and the line segment connecting the cross section center and CER center were used to compute $d_{B L}$. 


\section{CHAPTER III}

\section{EXPERIMENTAL VERIFICATION OF \\ THE NUMERICAL METHOD}

In order to verify the numerical method, a spherically shaped left ventricular model was built based on all the assumptions of the numerical model. The velocity field of the cross section alone the long axis were measured using Digital Particle Image Velocimetry (DPIV). The numerical procedure was carried out using FIDAP with the experimental boundary conditions as input.

\section{II.1 Experimental Setup}

Figure 3.1 shows the details of the experimental set up. A transparent latex balloon with a single outlet was used to simulate the left ventricular sac during systolic stage. Before contraction, the diameter of this balloon was dilated to approximately $8 \mathrm{~cm}$. The radius of the outlet was $3.3 \mathrm{~cm}$ which is approximately $40 \%$ of left ventricular chamber's radius (Gonzalez, 1995). A 0.5-centimeter grid was plotted on the right side wall of the rigid box. These grids were used to determine the pre-contraction left ventricular 
diameter and the position of the laser sheet. The left ventricle contracted and dilated passively by the pressure difference between the inside and outside of the left ventricle. When both valves are closed, a pressure difference can be built up by pressing the air into both water reservoirs using hand pumps. The small pressure difference could also be adjusted by changing the liquid level of the two reservoirs.

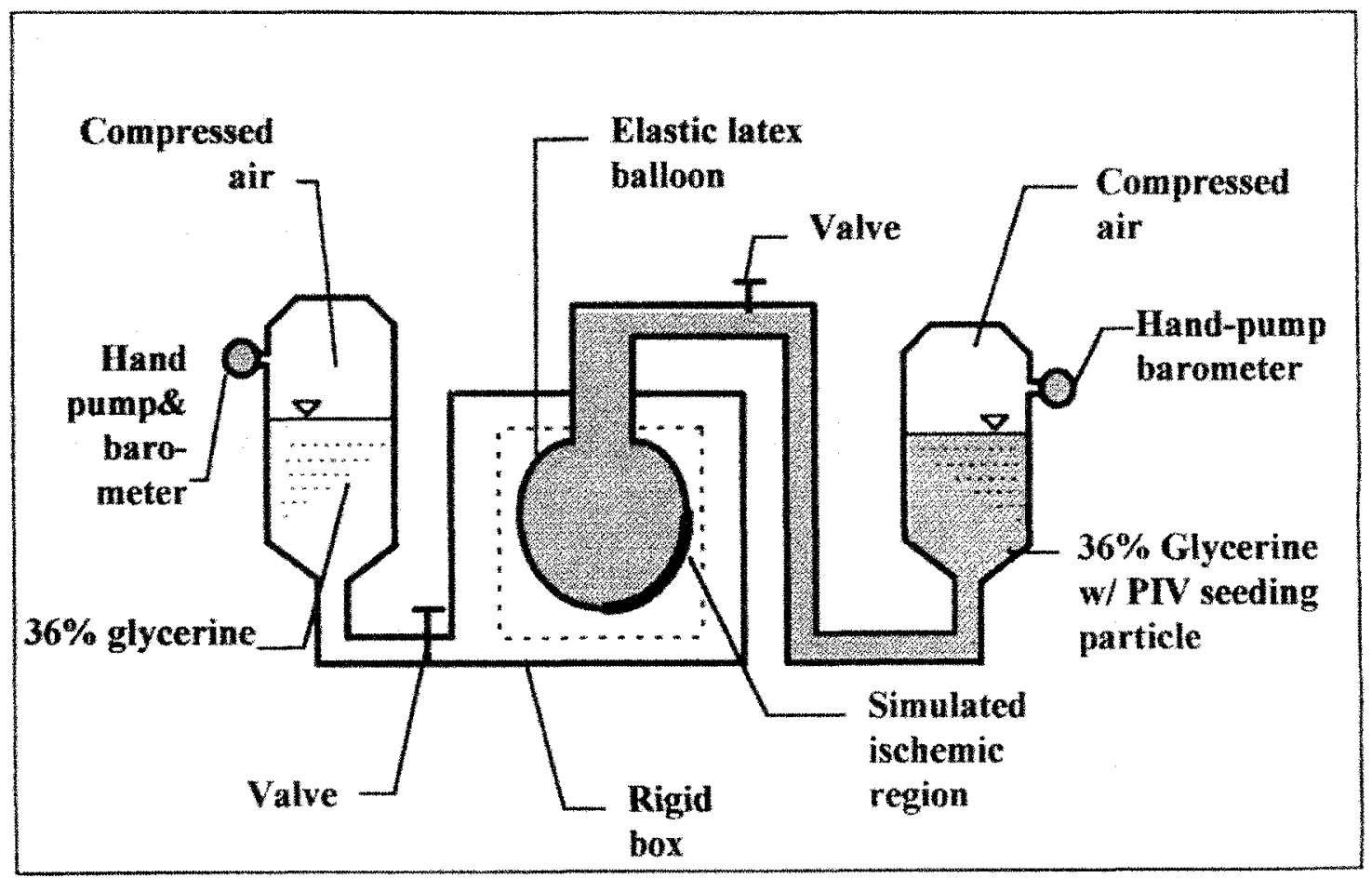

Figure 3.1 Experimental setup (dotted square indicates the test section)

The same fluid was used in both reservoirs: $36 \%$ glycerine by volume in saline. This solution matches both the density and the viscosity of human 
blood. PIV seeding particles were put in the fluid inside the left ventricle for the flow visualization and velocity fields measurements. These are OPTIMAGE seeding particles. They have a specific gravity of $1.0+/-0.02$ with wider distribution in sizes up to 250 microns. However nearly $80 \%$ of them have a diameter under 50 micron.

One normal case and one hypokinesis case was simulated. A thin layer of transparent Silicon glue was put on the pre-decided ischemic region to increase the stiffness of the left ventricular wall, so as to decrease the contraction of this region. Velocity measurements at the cross sections of a long axis plane for both cases were taken. For the hypokinesis case, the measured plane also went through the center of ischemic region.

\section{III.2 Digital Particle Image Velocimetry (DPIV) system}

DPIV is a laser software system which utilizes a non-intrusive technique that permits the mapping of instantaneous two-dimensional flowfields. Figure 3.2 shows the components of a DPIV system. A laser beam was generated from the laser generator, and converted to a thin sheet by the optic lens. In the present study, a 300 MV Argon laser was utilized. The laser sheet went through a plane of the test cross section of the flow. PIV 
beads in the flow field are illuminated by the laser and act as tracers which are captured by a CCD imaging camera with a maximum resolution of 486 $\mathrm{X} 1134$ pixels. The entire frame is transferred to a read-out buffer within 2 ms; thereafter, the sensor can be exposed again. A total of eight images can be saved in the buffer at the same time.

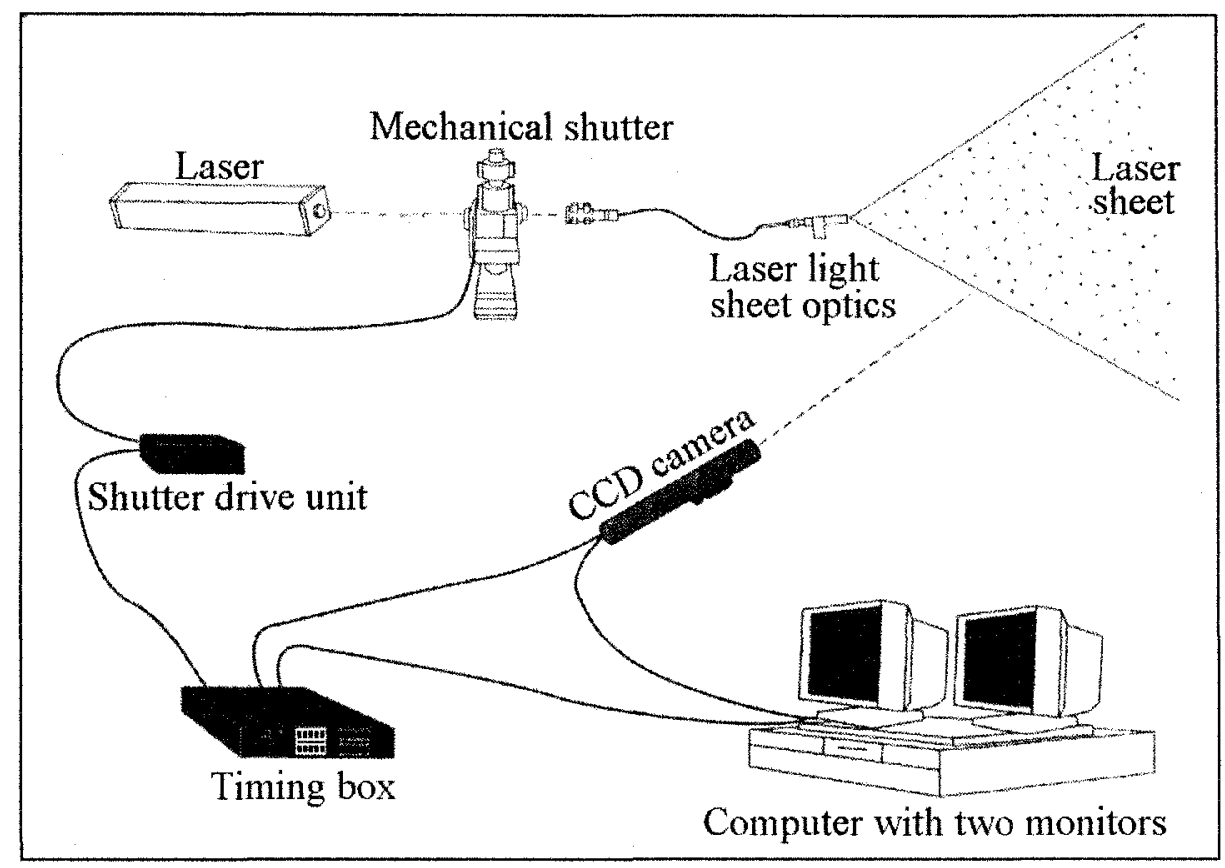

Figure3.2 DPIV system components

Before the laser beam was converted to a laser sheet, it went through a mechanical shutter which is controlled by a timing box and a shutter drive unit. The timing box could be programmed to control the mechanical shutter to chop the laser light at the camera's frame rate to prevent a performance 
degrading streaking of the particle images in the camera during the exposure. For the present study, $33 \mathrm{~ms}$ period between exposures was utilized.

A user friendly software FLOWGRABBER ${ }^{\mathrm{TM}}$ is provided with this system. It subsampls the two sequential digitized images with a variable size interrogation window, and computes the cross correlation of the 2 image samples with a Fourier analysis technique. The computation originally resulted in a displacement field of the tested cross section. Other associated kinetic fields such as velocity field, stream function, divergence, strain and vorticity, were derived from the displacement via finite differentiation and integration. Various options are allowed for data presentation in the form of vector plots, contour plots and ASCII files.

\section{III.3 Experiment Procedure}

As we mentioned before that two sets of experimental measurements were taken, one for normal left ventricle and one for hypokinesis left ventricle. For each case, the same measurements were repeated seven times for seven evenly distributed cross sections along the short axis. Due to the reason that there is no electronic equipment to trigger the flow and the 
DPIV system at the same time, the best way to capture the start point of the flow is to set off the DPIV system first then open the valves. Eight images that were captured continuously. Among these eight images, the last one captured the stationary flow and the first one captured the moving flow were saved to be further processed. There are a total of fourteen pairs of images for each left ventricle.

The contours of the left ventricle on all images were digitized by the DPIV built-in function. The images of the center cross section were also processed to get the velocity field.

The repeatability of the experiment was assumed by the following. Since the experiment set up is a closed semi-loop, the mass of the fluid inside the loop was constant. The rigid box and tubes kept the boundary conditions unchanged. The original fluid level of each reservoir was marked and the barometer reading was recorded before the first measurement was taken. Once the fluid was pumped back to its original position and the barometer was pumped to the same reading, the pressure boundary condition was the same. Therefore the pressure boundary condition was also constant. The fluid inside the left ventricle is also driven by the elastic force of the balloon itself. Since latex is an elastic material. The size of the 
pumped-up left ventricle is within its elastic limits. We can safely assume that its elastic characteristic has no change during the time period of each sets of the measurement that usually took less than one hour.

\section{III.4 Geometry Reconstruction}

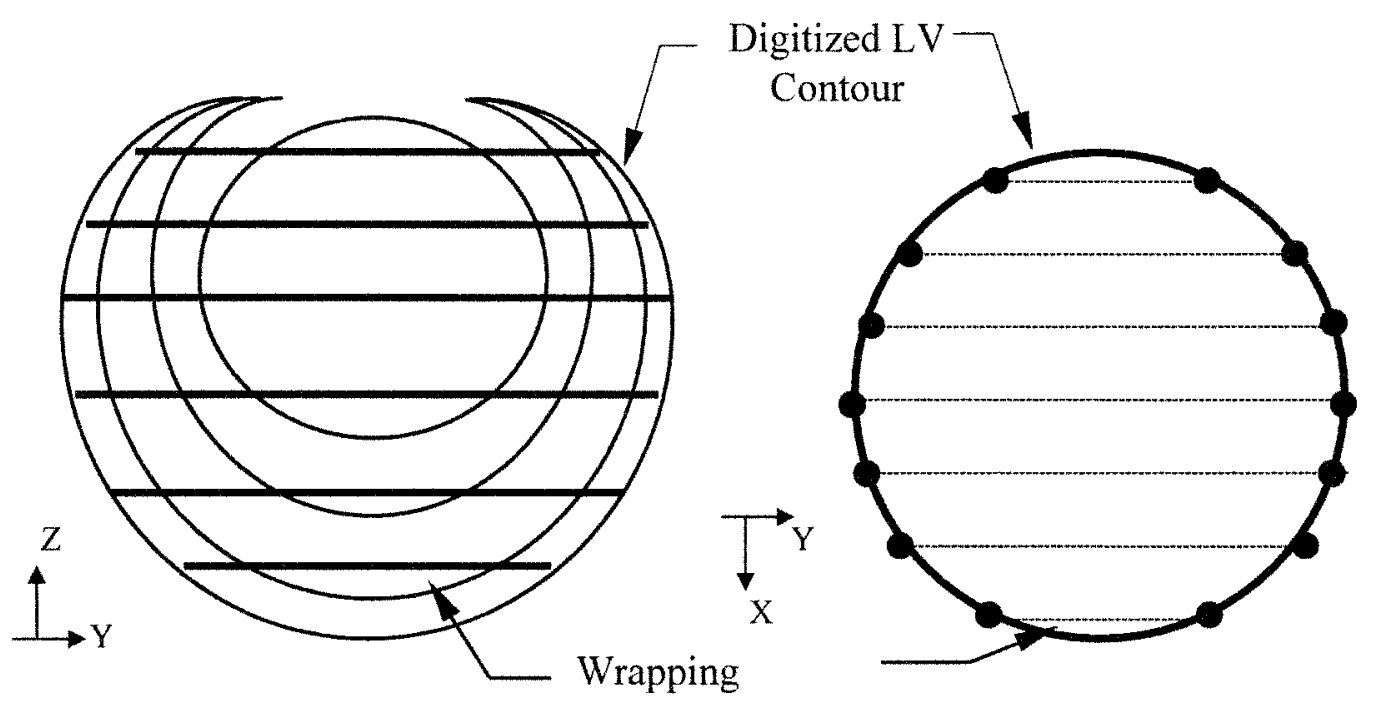

Figure3.3 Geometry Reconstruction

Seven digitized parallel vertical curves were used to reconstruct the whole geometry. These curves were then "wrapped" by two hundred horizontal curves. On each horizontal plane, two points on each vertical curve were digitized. Four to fourteen points were digitized for each horizontal cross section. Since the shape of the left ventricle was very close 
to a sphere. Every three consecutive points were fitted by an arc segment.

The segments formed a closed curve (figure 3.3). Two hundred points were digitized on each horizontal closed curve. These points were input to FIDAP to build the numerical geometry.

The mesh algorithm and boundary condition computation are the same as the ones for the human left ventricle model as we introduced in the previous chapter. 


\section{CHAPTER IV}

\section{RESULTS AND DISCUSSION}

\section{IV.1 Comparison of the numerical and experimental results}

The numerical results were qualitatively and quantitatively validated by the experimental measurements. Figure 4.14 to figure 4.19 are the comparison of experimental and numerical results for normal and ischemic left ventricles. For each case, the velocity field and two velocity components on two specific lines were compared. We arbitrarily chose one line on the upper half and one on the lower half of the cross section.

Velocity vector plots show a good qualitative agreement between the experimental and numerical results. The flow is symmetric with respect to the long axis for the normal left ventricle. For the ischemic case, the flow was generally directed towards the simulated ischemic region on the right lower part of the left ventricular wall.

A detailed comparision of the velocity on two specific lines of the cross section, a difference between the numerical and experimental results.

Compared to the numerical results, the $\mathrm{Z}$ component profile of the DPIV measurements are flat, especially for the curve near the bottom, with lower 
value in the middle and higher values at both ends. This can be explained by the fact that the experiments are transient with an actual contracting boundary. The measurements were taken within one second after the flow started to move from the stationary state. On the other hand, the numerical simulation solved the equations of steady flow, which means the flow is fully developed in time. The velocity difference on both ends of the curve can be explained by the difference between the real boundary condition and the assumed boundary condition we imposed on the numerical model. Also when we approach to the outlet, the effect of the boundary condition becomes smaller, so the two curves become more similar.

Figure 4.20 is the comparison of the CER between the numerical and experimental normal and ischemic LV. Since only two-dimensional data were available for the experimental results, the CER of the numerical model was calculated using two-dimensional formula for the corresponding plane. The graphs show that the CERs of the numerical models are narrower than that of the experimental models.

Although the above differences exist, there is still some agreement between the numerical and experimental results. For the normal left ventricle, the peak stays in the middle for both numerical and experimental 
measurements. For ischemic left ventricle, the peaks of the velocity profile apparently shifted to the right with approximately the same amount for the experimental and numerical cases. Each of the $\mathrm{Y}$ component profile curves reaches zero at the same or close to the same position as well.

Based on the above fact, it is believed that the numerical model can produce a CER that accurately reflects the flow development in the left ventricle.

\section{IV.2.1 Flow patterns for various time steps of normal and ischemic left}

\section{ventricles}

In this section, the velocity patterns of all time steps of the normal and ischemic left ventricles are discussed. To better visualize the threedimensional data, only the plots of the cross section, which contains the characteristic flow, were presented. Since both the geometry and the boundary conditions are symmetric with respect to the $\mathrm{Y}-\mathrm{Z}$ plane, the velocity vectors of the cross sections that aligned with $\mathrm{Y}-\mathrm{Z}$ plane are presented. Since the $\mathrm{Z}$ component of the velocity is the dominant one among all three velocity components. The contour plots of the $\mathrm{Z}$ component 
on the same cross section are also presented to help us to get a better look at the flow pattern.

Figures $4.1 \mathrm{a}$ to $4.1 \mathrm{c}$ are the velocity vectors and the $\mathrm{Z}$ component contour lines of each time step of the normal left ventricle. Figures $5.2 \mathrm{a}$ to $5.2 \mathrm{~b}$ are the velocity vectors and the $\mathrm{Z}$ component contours lines of all the time steps of the ischemic left ventricle.

It is clearly shown that the contour lines of the normal left ventricle for all time steps are shaped like narrow wedges pointed downwards. The lowest point of all the contour lines appeared approximately in the middle. This means that the high velocity region is in the middle of the cross section. Flow has been highly accelerated from the boundary to the center. The contraction is strong and symmetric.

Similarly shaped contour lines, with the lowest point in the middle, only appeared at the second time step (IT2) for the ischemic left ventricle. The contour line of the first time step (IT1) and third time step (IT3) are much more flat than that of the normal left ventricle. The lowest point of the contours lines shift to the right wall instead of staying in the middle. This means that the flow had not been accelerated much from the wall. The 
contraction is weak and non-symmetric. There is no apparent flow pattern for the last time of the ischemic left ventricle.

Table 4.1 and table 4.2 present the computed Reynolds number of each time step of the normal and ischemic left ventricle. The peak value of the two Ventricles are 3126.22 and 1317.69.

\section{IV.2.2 Flow pattern of simulated ischemic left ventricles}

As mentioned in the previous section, an ischemic area was imposed on the normal left ventricle with controlled abnormal wall motion to simulate the hypokinesis $(\mathrm{F}=0.5,0.3,0.1)$, akynesis $(\mathrm{F}=0.0)$ and dyskinesis $(F=-0.1,-0.3)$. For the simulations with the ischemic area on the right wall of the left ventricle, the same cross sections as the ones used in the previous section are presented. For the simulations with the ischemic region on the back wall, the $\mathrm{X}-\mathrm{Z}$ plane was chosen to present the velocity vector and $\mathrm{Z}$ component contour lines.

Figure $4.3 \mathrm{a}$ to $4.5 \mathrm{c}$ present the velocity vectors and twenty $\mathrm{Z}$ component contour lines of all the simulations. The corresponding contour lines are of the same value. 
The velocity vector plots qualitatively show that the flow around the ischemic region was directed to the ischemia more and more with decreases of F.

The $\mathrm{Z}$ component contour plots gave us a clearer look at the flow dynamics changes with the change in size and severity of the ischemic region. Almost all the contour lines except the ones near the outlet were affected by the ischemia. When $\mathrm{F}$ is larger than 0 , all the contour lines are attracted to the ischemic wall. Near the ischemic region, the contour lines become more and more dense with decreases of $\mathrm{F}$. When $\mathrm{F}$ is lower than 0 , the contour lines appear further away from the ischemic wall. The existence and the magnitude of the $\mathrm{Z}$ component at the boundary cause this behavior of the contour lines.

With the decrease of $F$ from 0.5 to -0.3 , the shape of the corresponding contour lines became more and more flat. The lowest point of the corresponding contour lines continually moves upward to the outlet. For the simulation with the large ischemic area, the contour lines gradually changed from one valley to two valleys.

Since we keep the Reynolds number the same for all cases, the outlet flow rate is the same. The change of the flow patterns show that with the 
severity of ischemia increased, the flow near the ischemic region contributed less and less to the outflow, while the flow of the unaffected regions contributed more and more.

The simulations with the ischemia on the side with two different sizes were compared. It is observed that with the same $F$, although the $\mathrm{Z}$ component contour lines of the simulation with large ischemia do not shift as much as the ones with small ischemia, their lowest point moved further upwards. The deformation of those contour lines is much bigger than that of the corresponding ones with small ischemia simulation.

\section{IV.3.1 CER and CER coefficient of normal and ischemic left ventricles}

As mentioned in the previous chapter, a clinical index Central Ejection Region (CER) as well as its quantitative version, the CER coefficient, would be used to estimate the global and regional performance of the left ventricle contraction. Figure $4.6 \mathrm{a}$ to $4.7 \mathrm{~d}$ present the CER of each time step of normal and ischemic left ventricles. Each CER is presented by two three dimensional views, a front view and a side view. Figure 4.11 and Table 4.3 presents the CER coefficient at each time step for the normal and 
ischemic left ventricles as well as the time-average value over the entire systolic period.

For all four time steps of the normal left ventricle, the CERs are solid, pillar shaped running continuously from the outlet to apex. Except the fourth time step, all the CERs were located in the middle of the left ventricular cavity, following the center line of the cavity very well, particularly the second and third time steps. The CER for the fourth time step shifted to the right wall and there appeared a little split at the end. This may attribute to the small area near the apex which has a weaker contraction compared to the unaffected areas.

Only the CER for the second time step of the ischemic left ventricle is similar to the CER for the normal left ventricle. For all other time steps, the CER was shorter and smaller in volume than the ones of the normal left ventricle. The shapes are thin and flat, and splitting to several branches, rather than staying in a solid pillar shape. Especially the last time step, the whole CER shrunk to a few very small pieces. Only a small section of the each CER followed the centerline, where most parts stayed along the left ventricular wall. 
Quantitatively the CER coefficient for the normal left ventricle ranged from 0.514 to 0.832 with a time-averaged value of 0.658 over systole. For the ischemic left ventricle, the CER coefficient ranged from 0.054 to 0.783 , with a time-averaged value over systole of 0.362 , which is about $55 \%$ of CER for normal left ventricle.

\section{IV.3.2 CER and CER coefficients of the simulated ischemic left}

\section{ventricles}

Figure $4.8 \mathrm{a}$ to $4.10 \mathrm{c}$ present a three-dimensional view, a front view and a side view of every CER for the simulated ischemic cases. The CER exhibited an apparent change with the change in ischemic region and $\mathrm{F}$.

For the hypokinesis cases, the lower part of the CER, corresponding to the ischemic region along the $\mathrm{Z}$ direction, had been pushed into the region which is surrounded by the ischemic left ventricular wall even with the mildest abnormal wall motion. The CER remained inside this region when $\mathrm{F}$ is larger than 0 . When $\mathrm{F}$ equals 0.5 , the lower part of the CER became concave and extended along the ischemic wall. The cross section of the CER changed from a circle to an arc. With the decrease of $F$, the concave CER split into three branches. With more decreases in $\mathrm{F}$, each 
branch became thinner and shorter, and moved closer and closer to the wall.

The middle branch moved towards the center of the ischemia whereas the other two branches moved closer to the corresponding boundary of the ischemic region.

When the abnormal wall motion became severer (akinesis or diskineses), the CER inside the ischemic region completely disappeared. There were still short branches of the CER above the ischemic region. There is insignificant difference among the CERs for the same ischemic region. With decreases in $\mathrm{F}$, the branches became slightly shorter and thinner, the split point moved slightly upwards.

Comparing Figure $4.10 \mathrm{~b}$ and $4.11 \mathrm{c}$, the CER along the long axis direction was affected when the ischemia was on the side than when the ischemia on the back of the left ventricular wall. This shows that the CER is more sensitive to the ischemia location when the ischemia is located on a cross section of a more regular geometry.

Table 4.1 and Figure 4.10 shows the CER coefficients of the simulated ischemic cases and the CER coefficient against $F$. With the same ischemic region, the CER coefficient decreased with a decrease in $F$. When F is zero, the curve became flat. With the same simulation factor, the 
simulation with the small ischemia on the side has the lowest CER coefficient, whereas the simulation with the large ischemia on the back has the highest CER coefficient. This is in partial agreed with Gonzalez. Figure 4.13 is the results based on spherical shaped model similar size and location of the ischemic region.

As discussed before, the CER tends to stay inside the region, which is surrounded by the ischemic wall. The larger ischemic wall surrounded a larger region than the small ischemic wall; so obviously, the CER for simulation with the larger ishemic region is closer to the centerline than the CER of the simulation with small ischemic region. We also discussed before that the ischemia on the side has a bigger affect on the CER than the ischemia on the back.

\section{IV.4 Comparision with Other Models}

These resulting flow fields from the current model agree with the results of others using axis-symmetric geometry models (Georgiadis et al. 1992 and Pelle et al. 1993) and more realistic geometry models (Peter et al. 1996 and Taylor et al. 1995), in that the flow was generally directed toward 
the long axis, and then out through the orifice for a normally contracting ventricle.

Compared to the models with Peter et al. and Taylor et al.'s model, both the vector plots and the magnitude images show that the flow converged toward the aortic valve from the entire ventricle. The maximum velocity occurred at the outlet of the ventricle. The result from Taylor et al. Also agrees that there is a definite difference in the geometric shapes and flow patterns in normal and ischemic hearts. The vector plot along the plane aligned with the long axis has a similar velocity profile as our results.

The result that CER is more sensitive with a more regular geometry $\mathrm{s}$ agrees with Gonzalez's work (1994). In his results, based on a spherical shaped model, the CER is very sensitive to the ischemia region.

Some observations are different from the previous models. Compared to the two-dimensional results by Silva (1991), the ischemia affects a larger region of the CER along the long axis direction. Compared to Gonzalez's (1994) results, a split of the CER is observed, and the CER is less sensitive to the location of the ischemic region. Most importantly, the current study gave a complete and a detailed look at some physical characters of the three dimensional CER. 


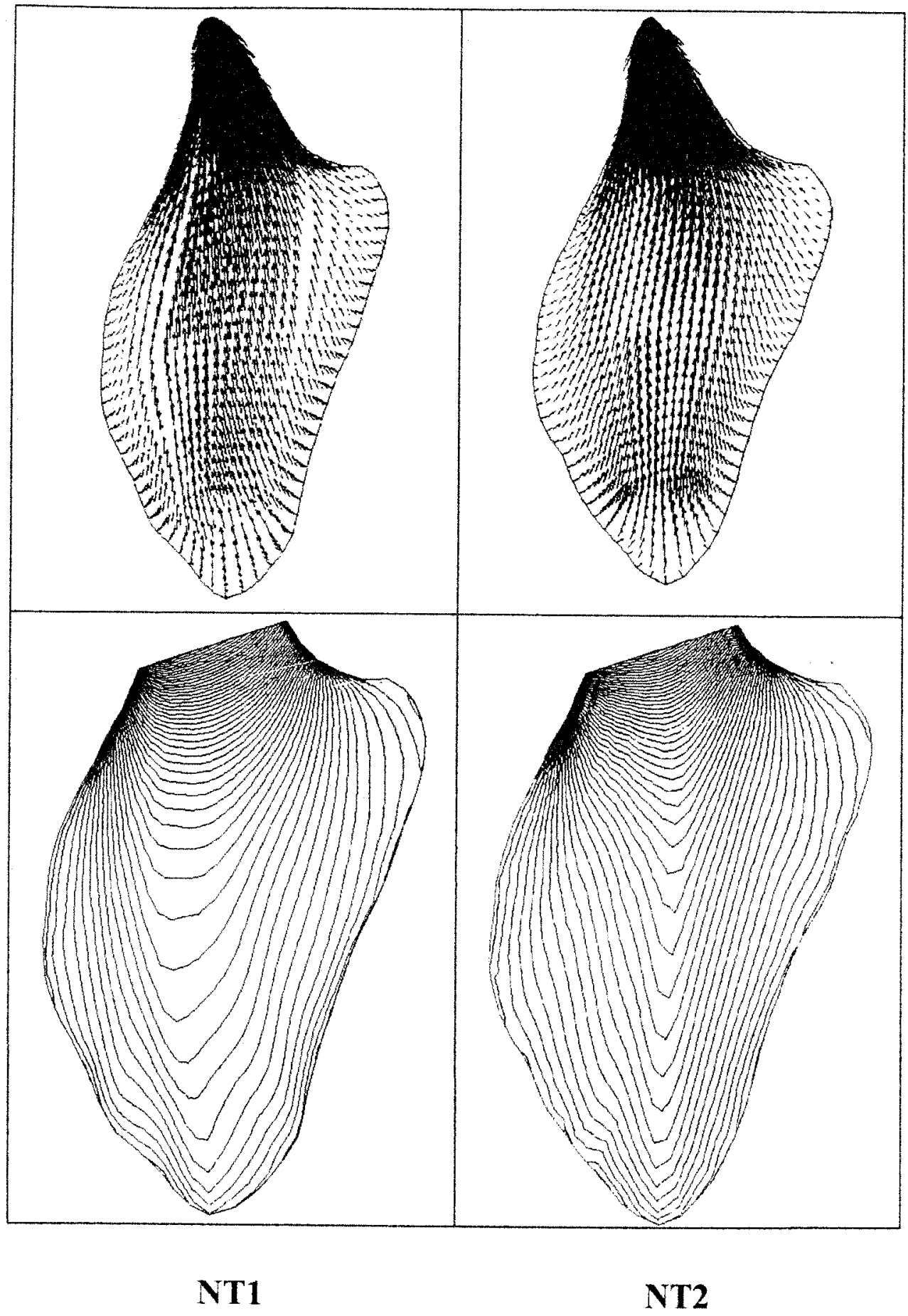

Figure 4.1a. Velocity \& Vz Contour of Normal left ventricle 


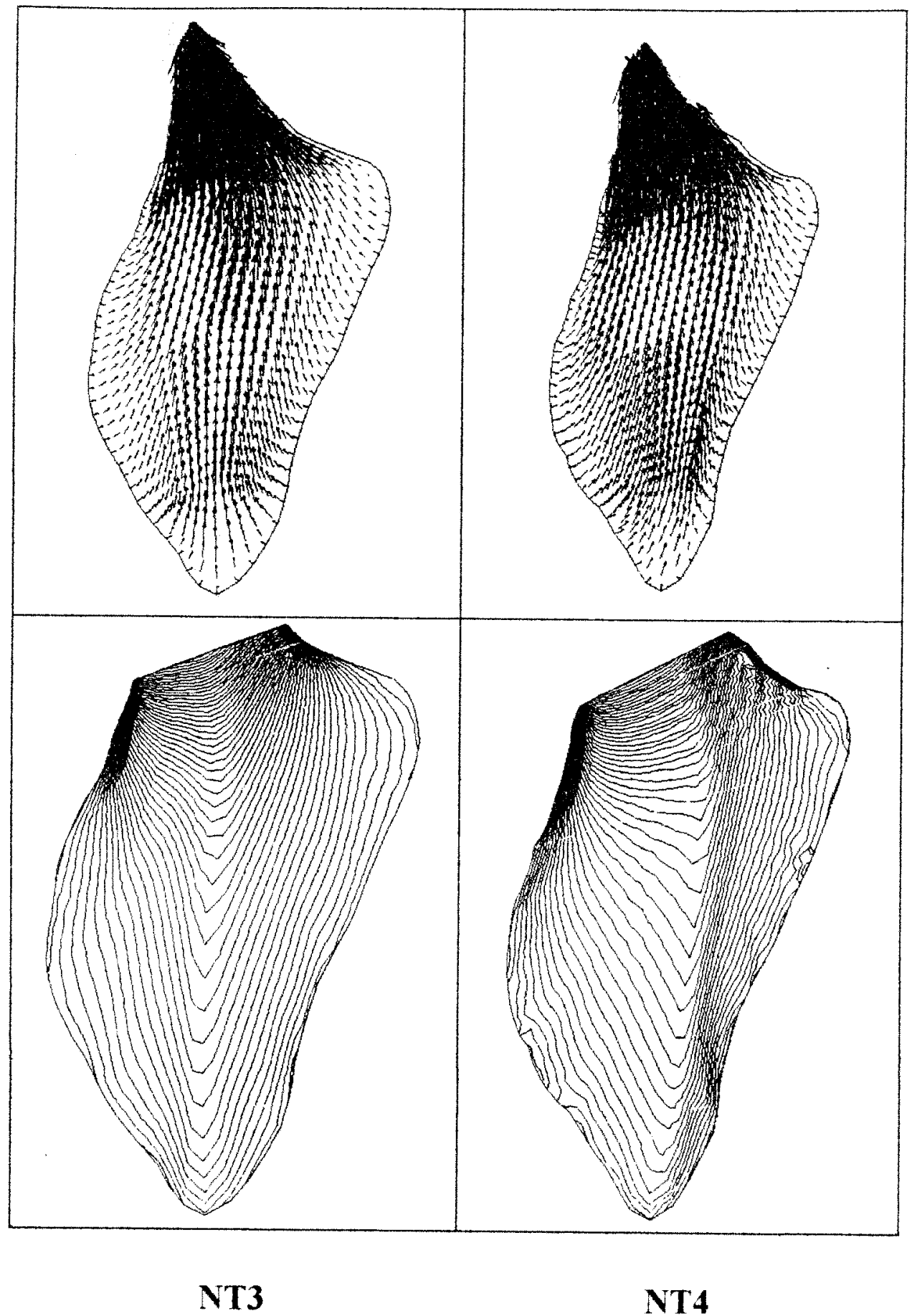

Figure 4.1b. Velocity \& Vz Contour of Normal left ventricle 


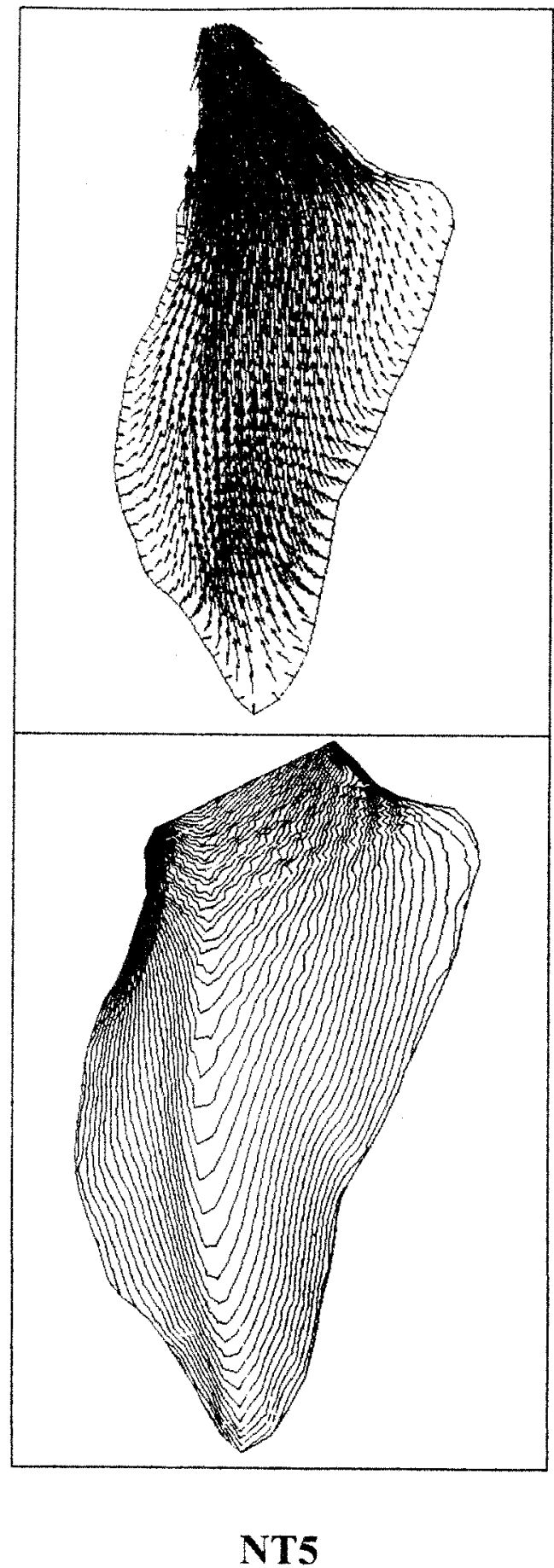

Figure 4.1c. Velocity \& Vz Contour of Normal left ventricle 


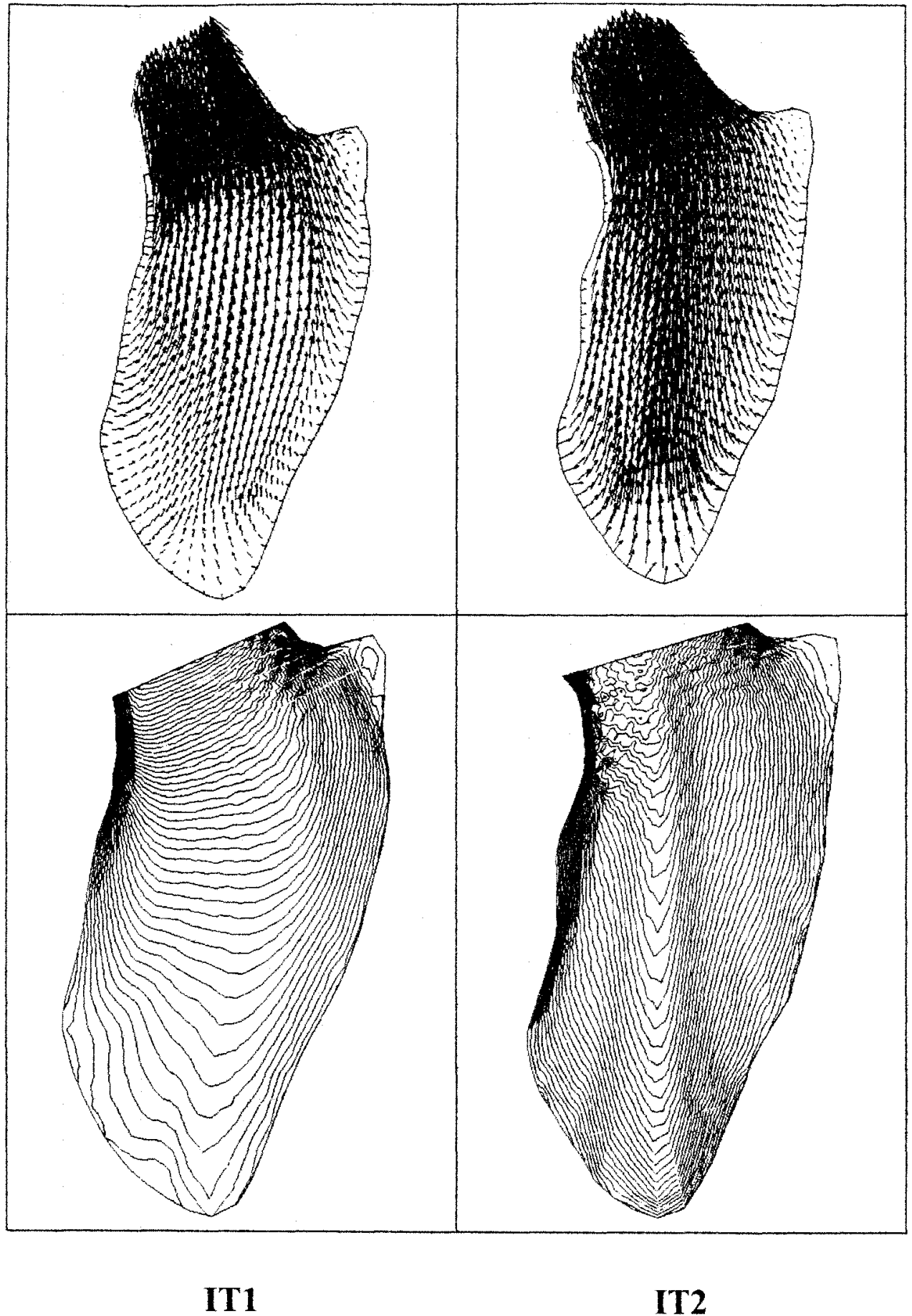

Figure 4.2a. Velocity \& Vz Contour of Ischemic left ventricle 


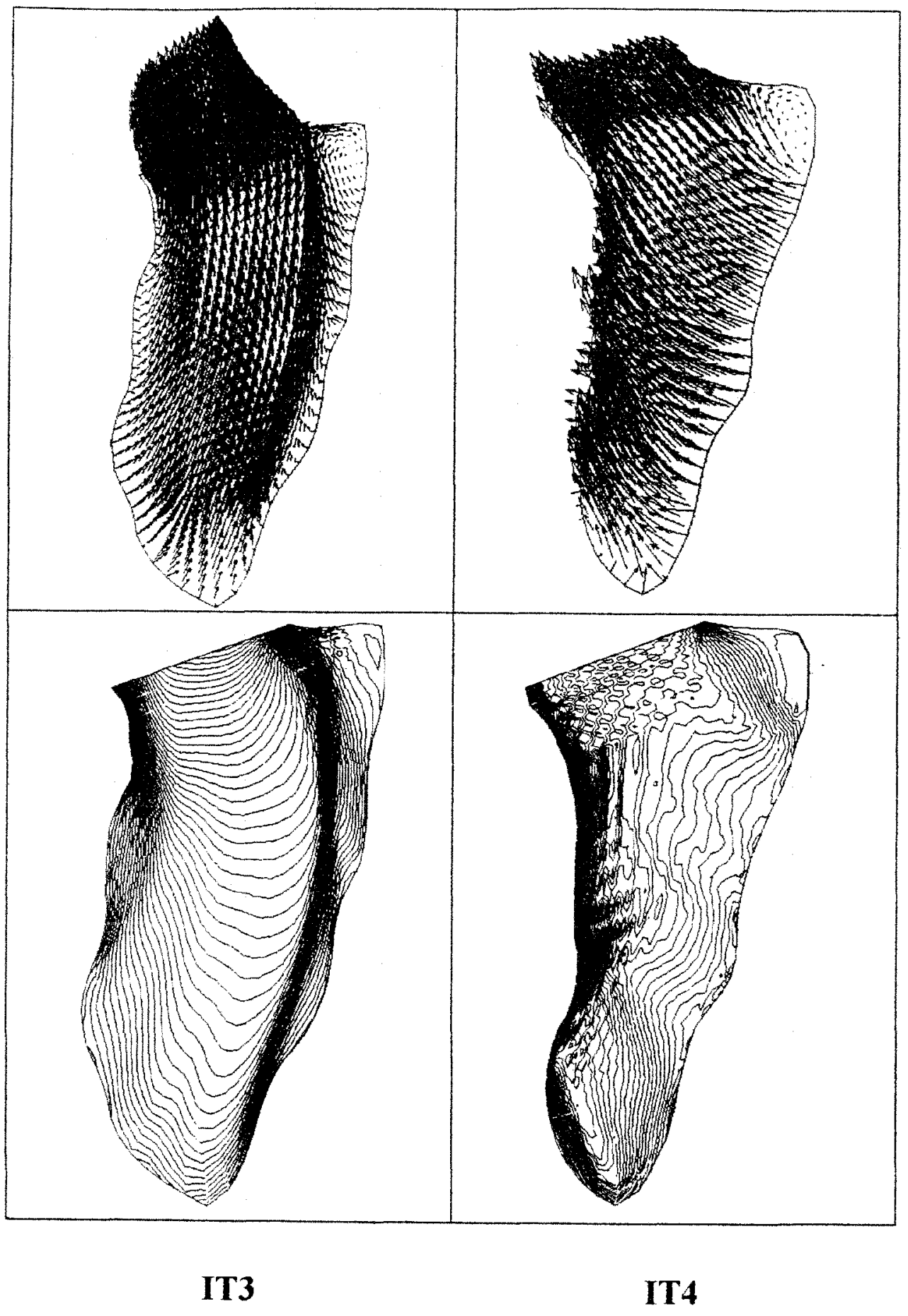

Figure 4.2b. Velocity \& Vz Contour of Ischemic left ventricle 


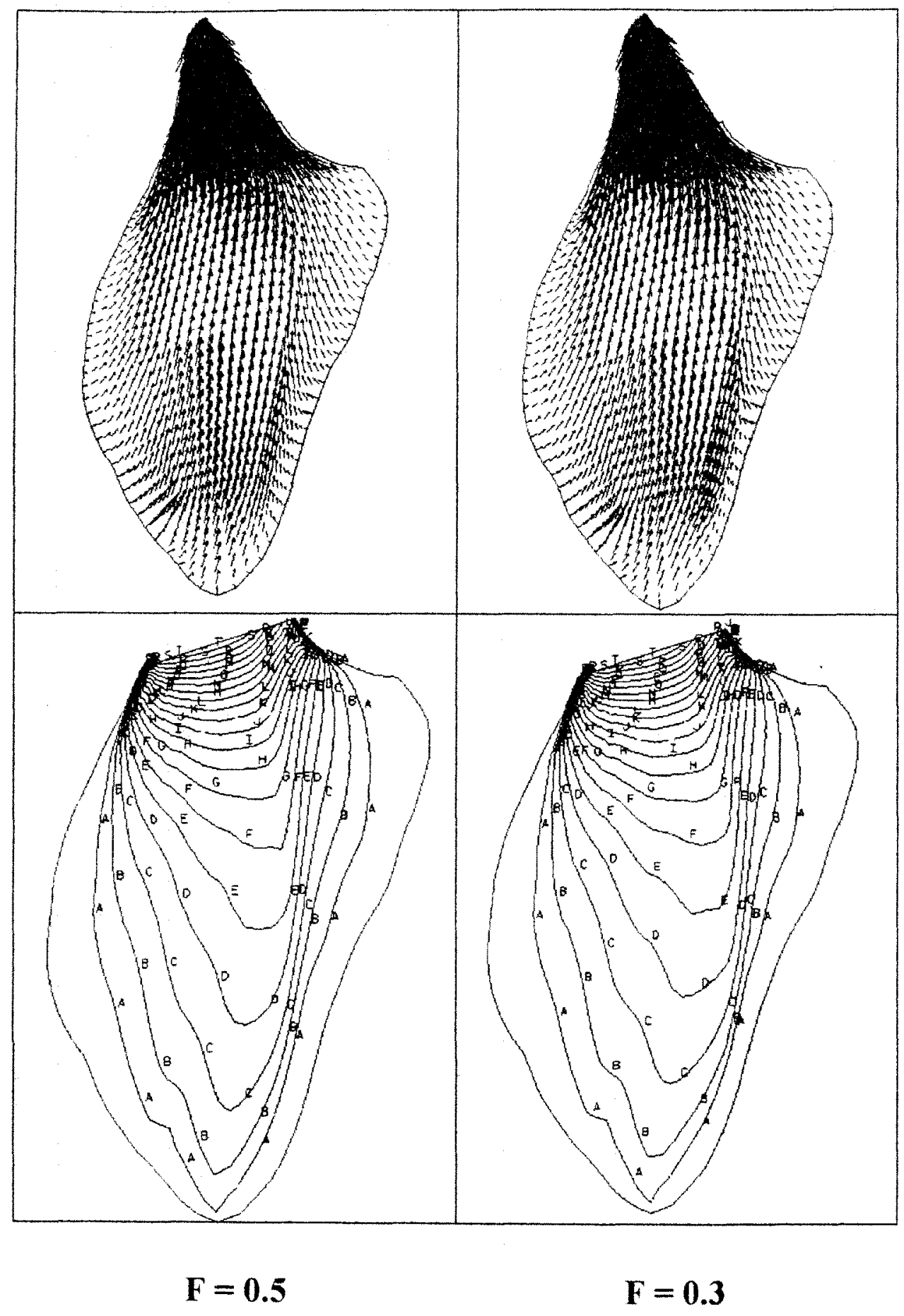

Figure 4.3a. Velocity \& Vz Contour of simulated ischemic left ventricle models small ischemia on the side 


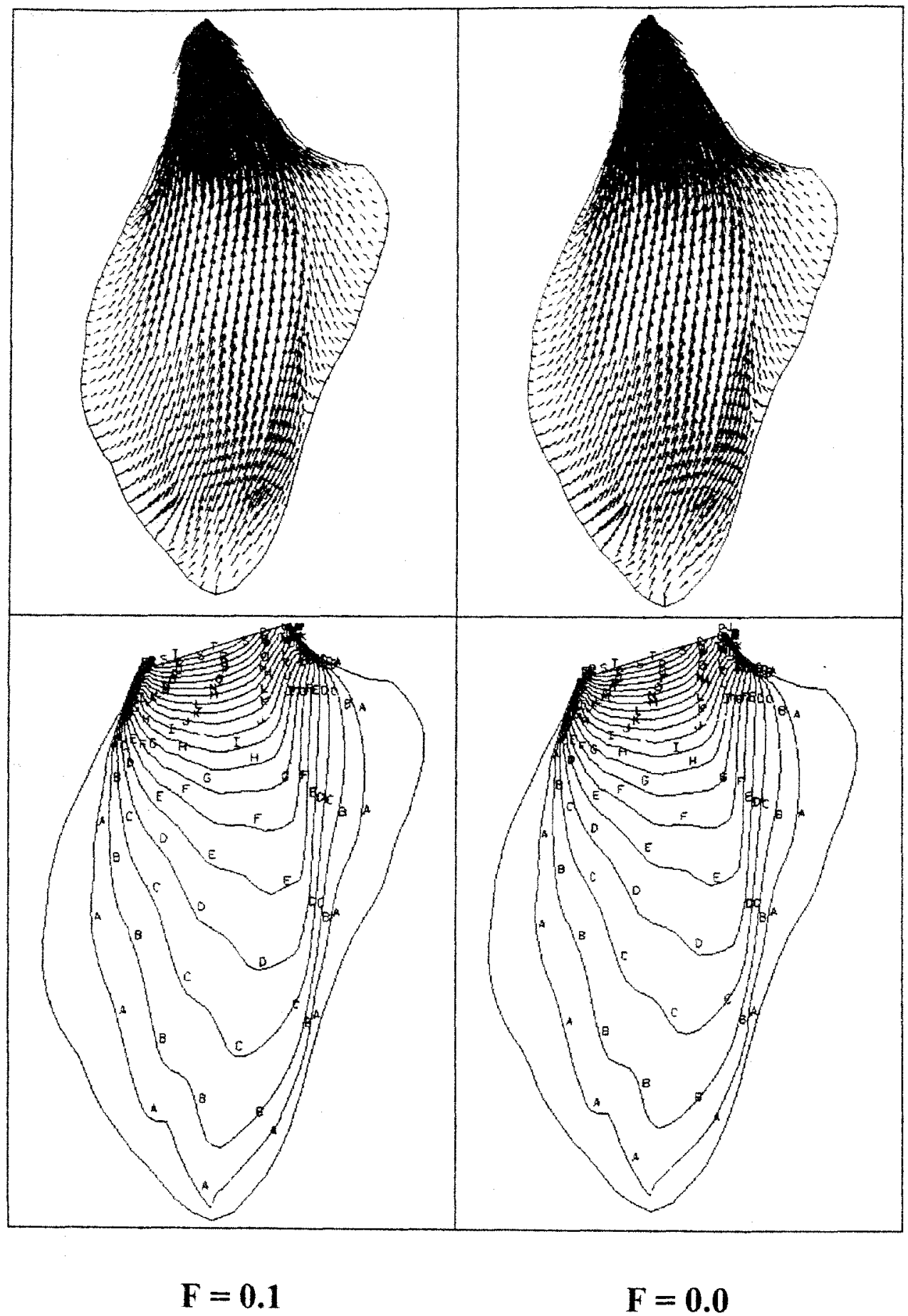

Figure 4.3b. Velocity \& Vz Contour of simulated ischemic left ventricle models small ischemia on the side 


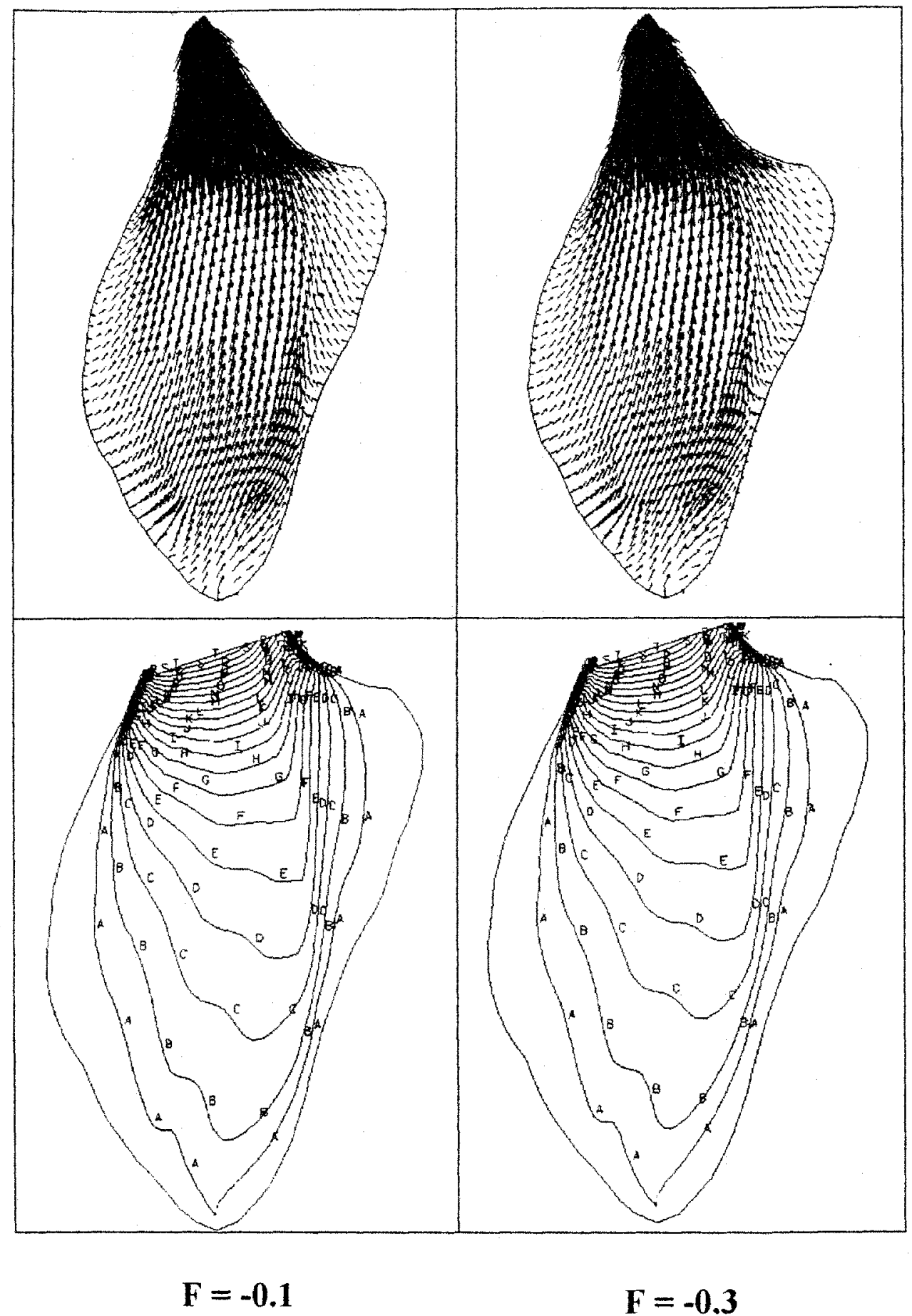

Figure $4.3 \mathrm{c}$. Velocity \& Vz Contour of simulated ischemic left ventricle models small ischemia on the side 


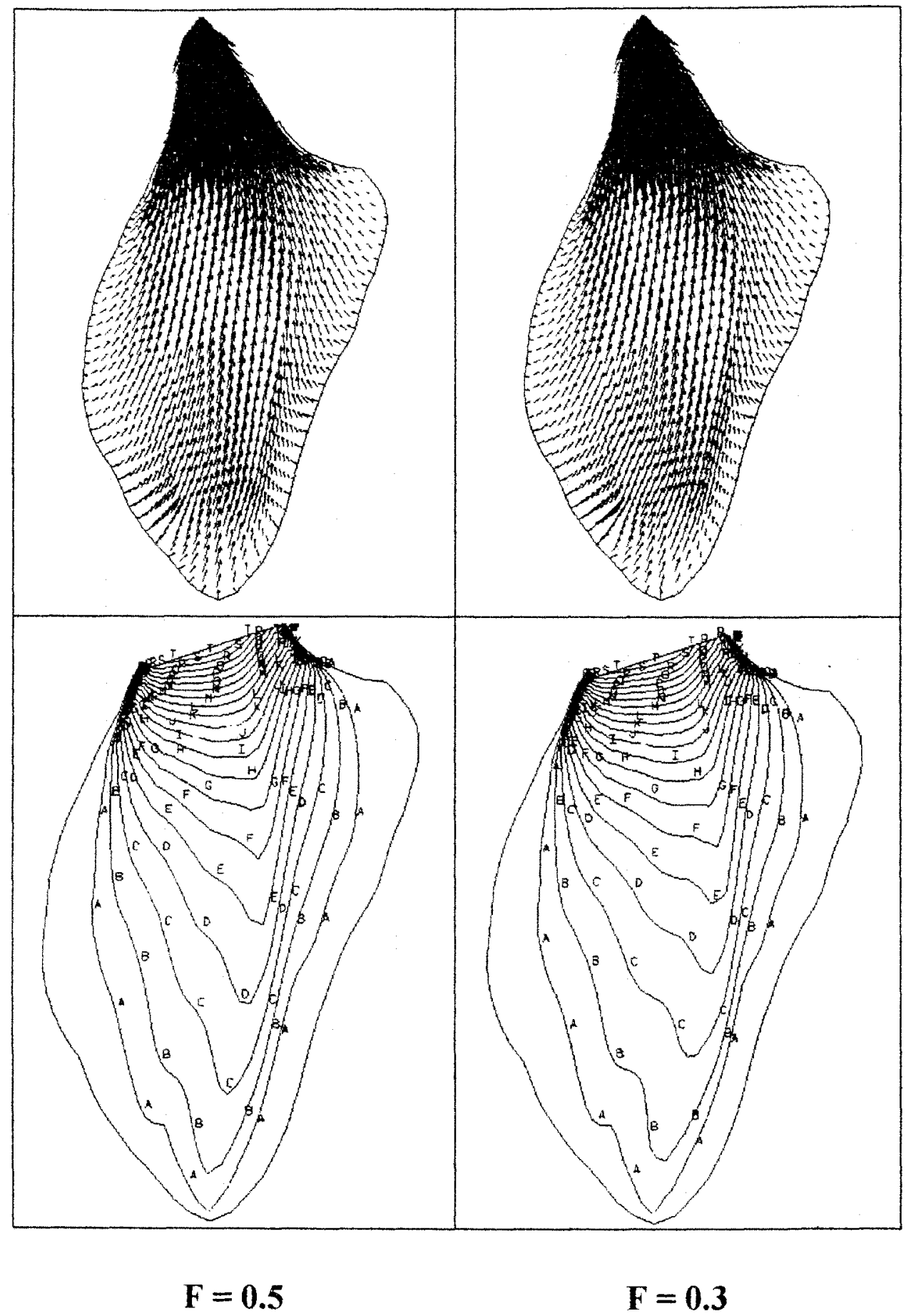

Figure 4.4a. Velocity \& Vz Contour of simulated ischemic left ventricles large ischemia on the side 


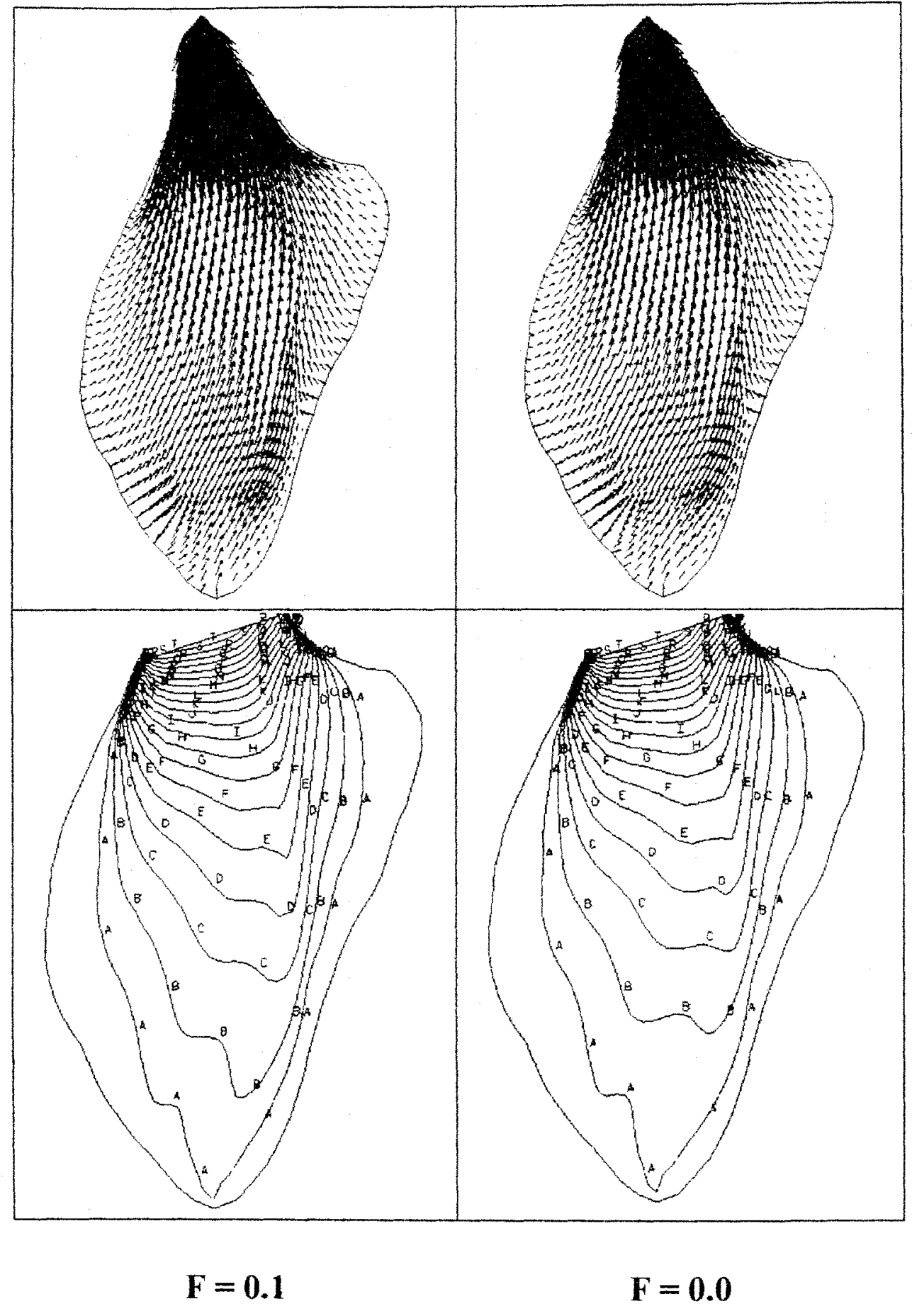

Figure $4.4 b$. Velocity \& Vz Contour of simulated ischemic left ventricles large ischemia on the side 


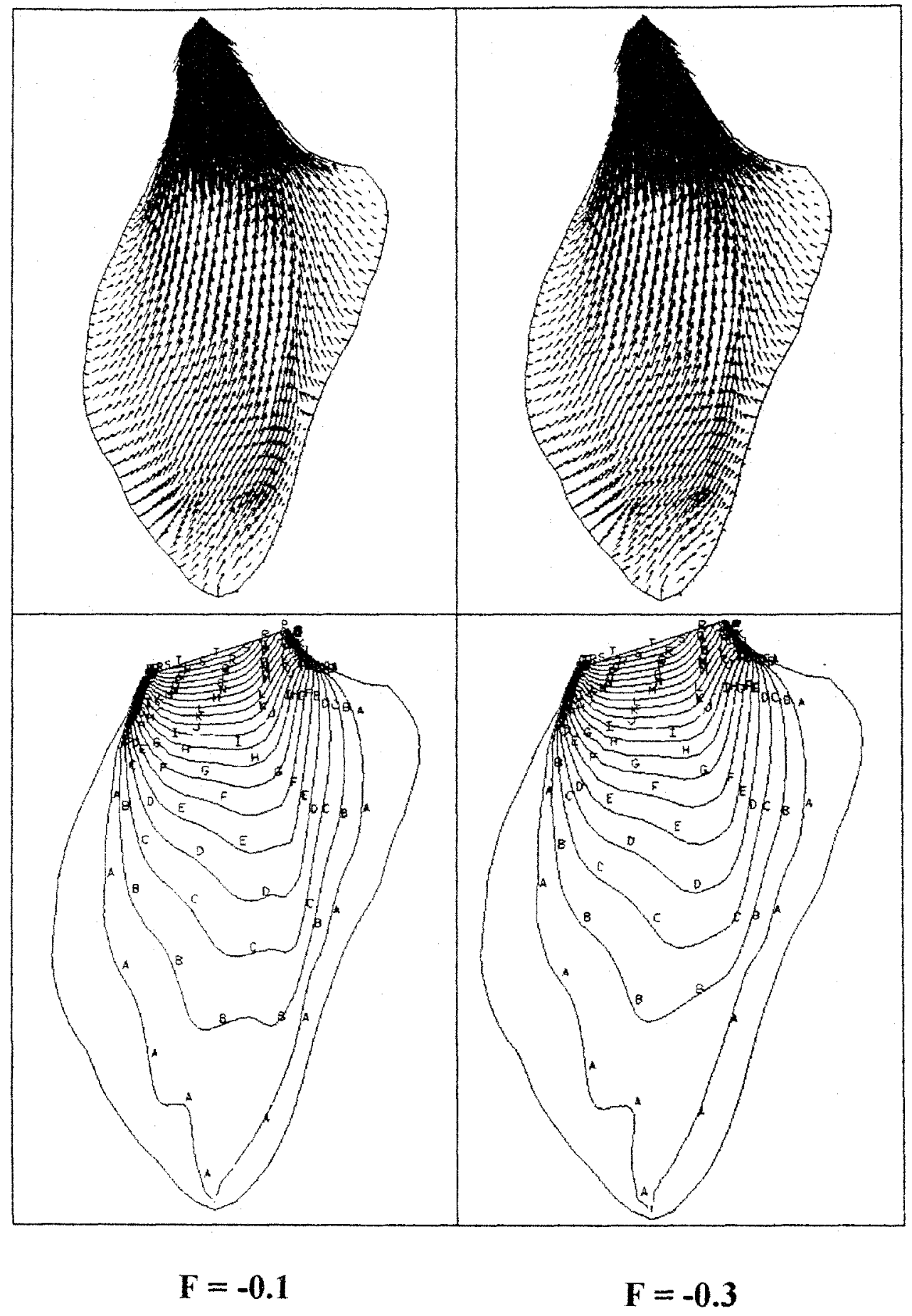

Figure $4.4 c$. Velocity \& Vz Contour of simulated ischemic left ventricles large ischemia on the side 


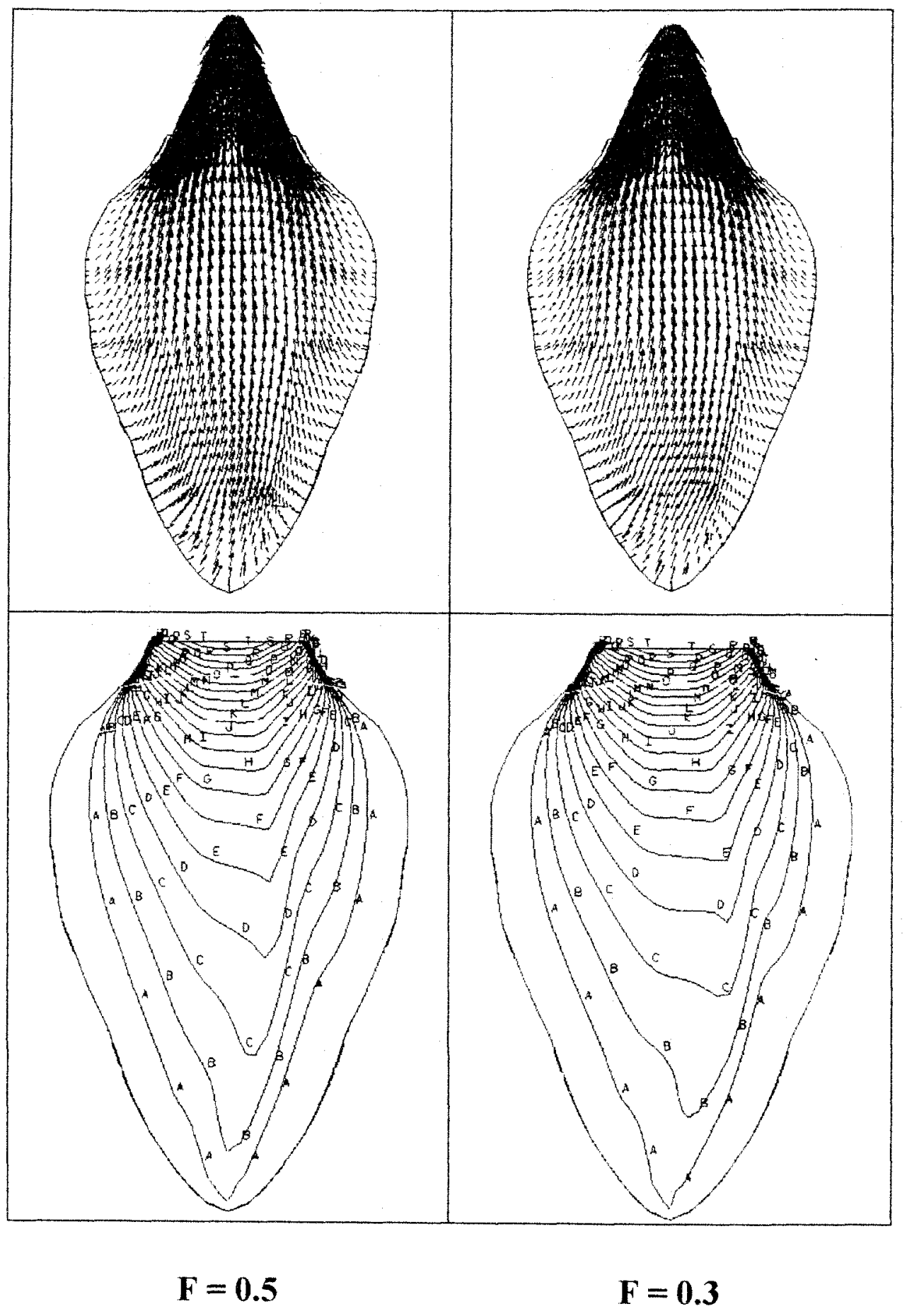

Figure 4.5a. Velocity \& Vz Contour of simulated ischemic left ventricles large ischemia at back 


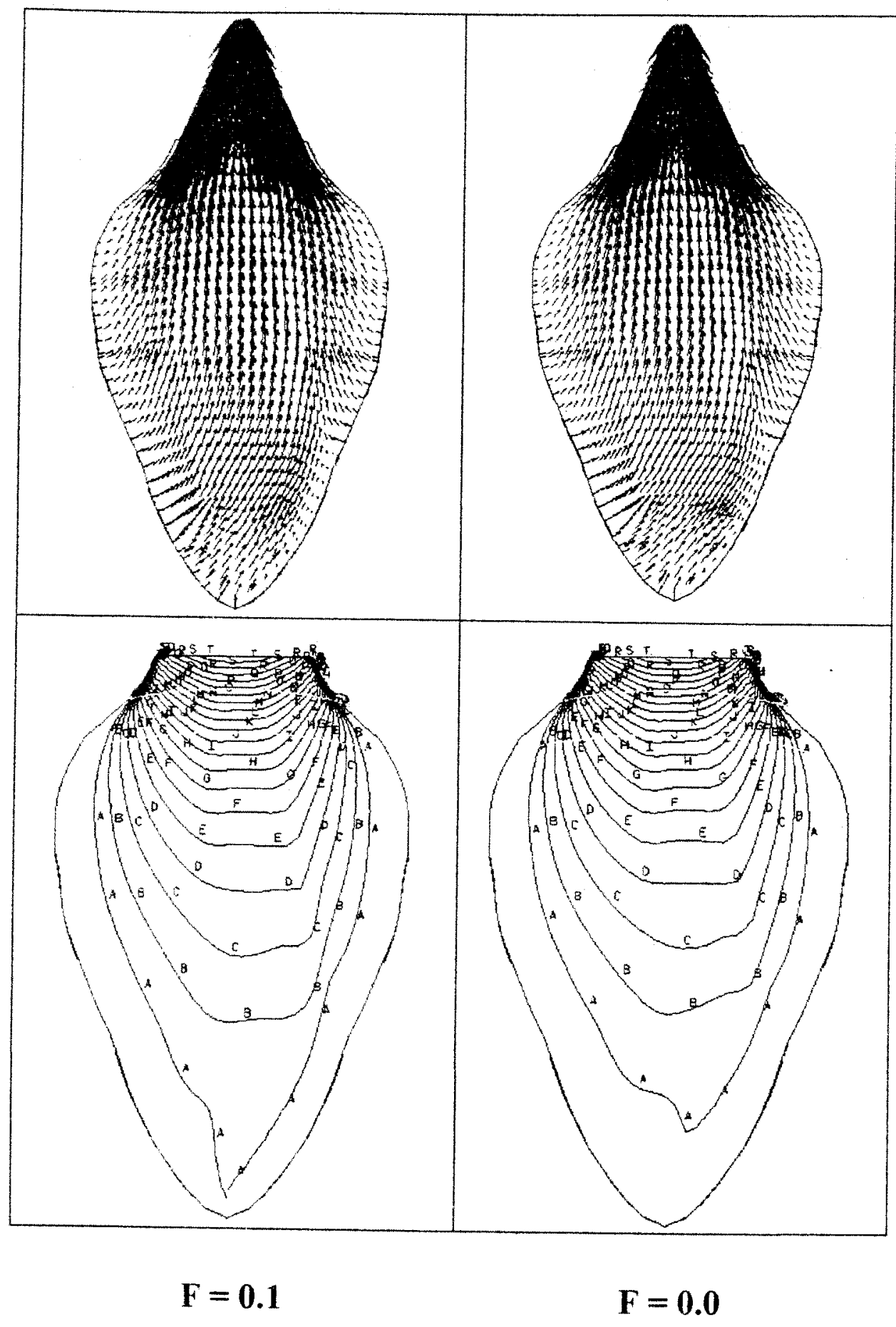

Figure $4.5 \mathrm{~b}$. Velocity \& Vz Contour of simulated ischemic left ventricles large ischemia at back 


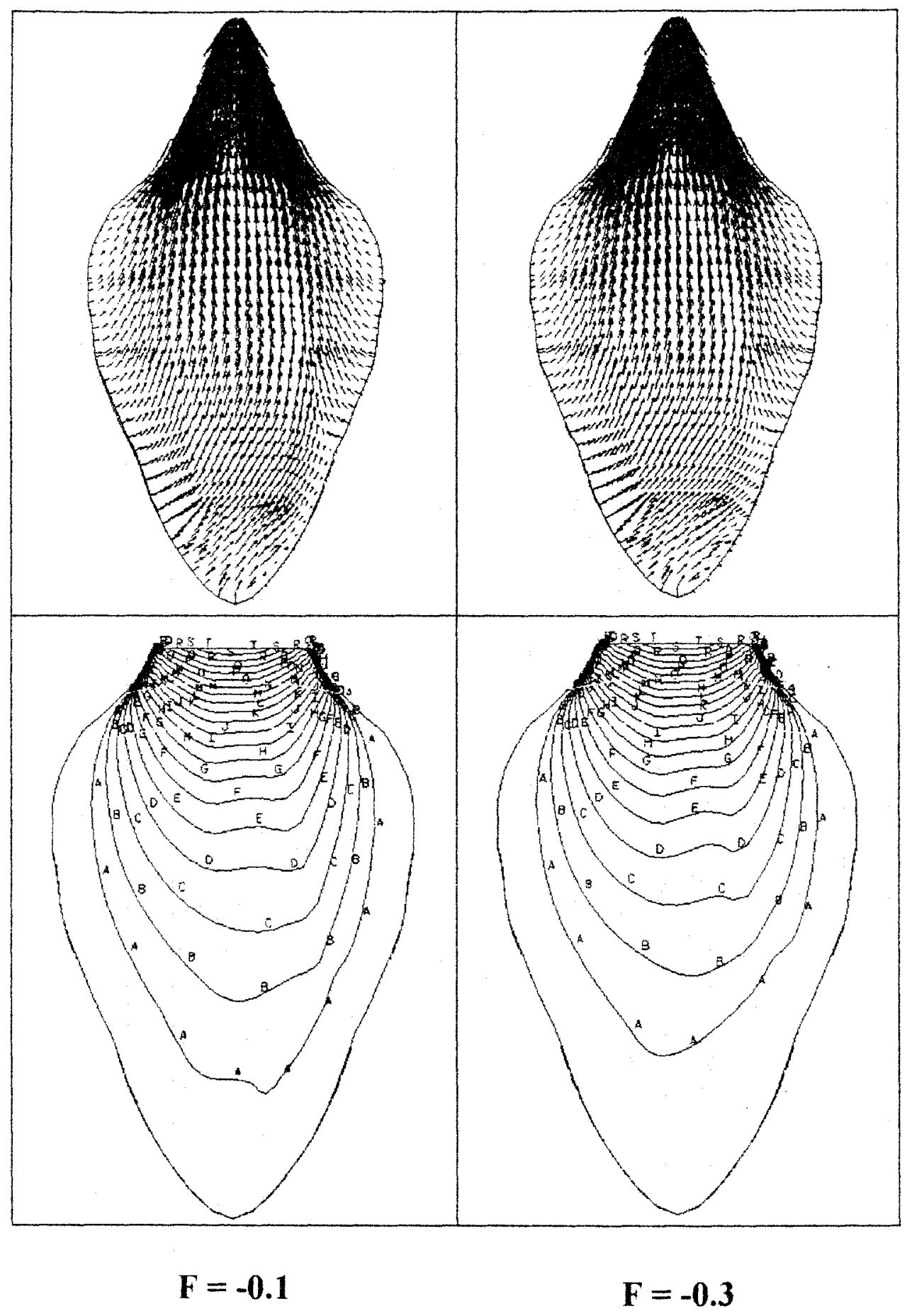

Figure $4.5 \mathrm{c}$. Velocity \& Vz Contour of simulated ischemic left ventricles large ischemia at back 


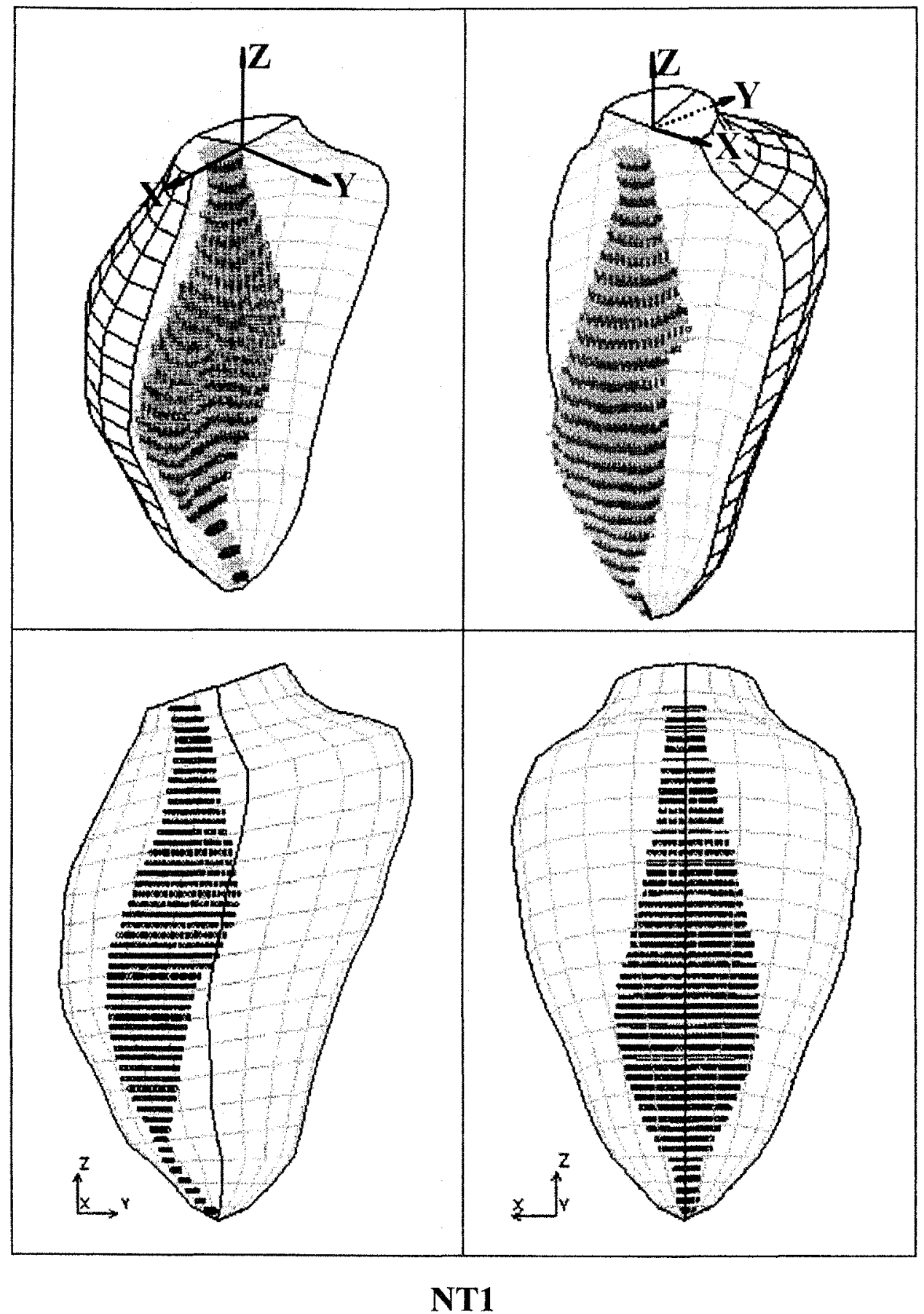

Figure4.6a Different views of the CER region inside the normal left ventricle $(\mathrm{NT} 1, \mathrm{t}=67 \mathrm{~ms})$ 


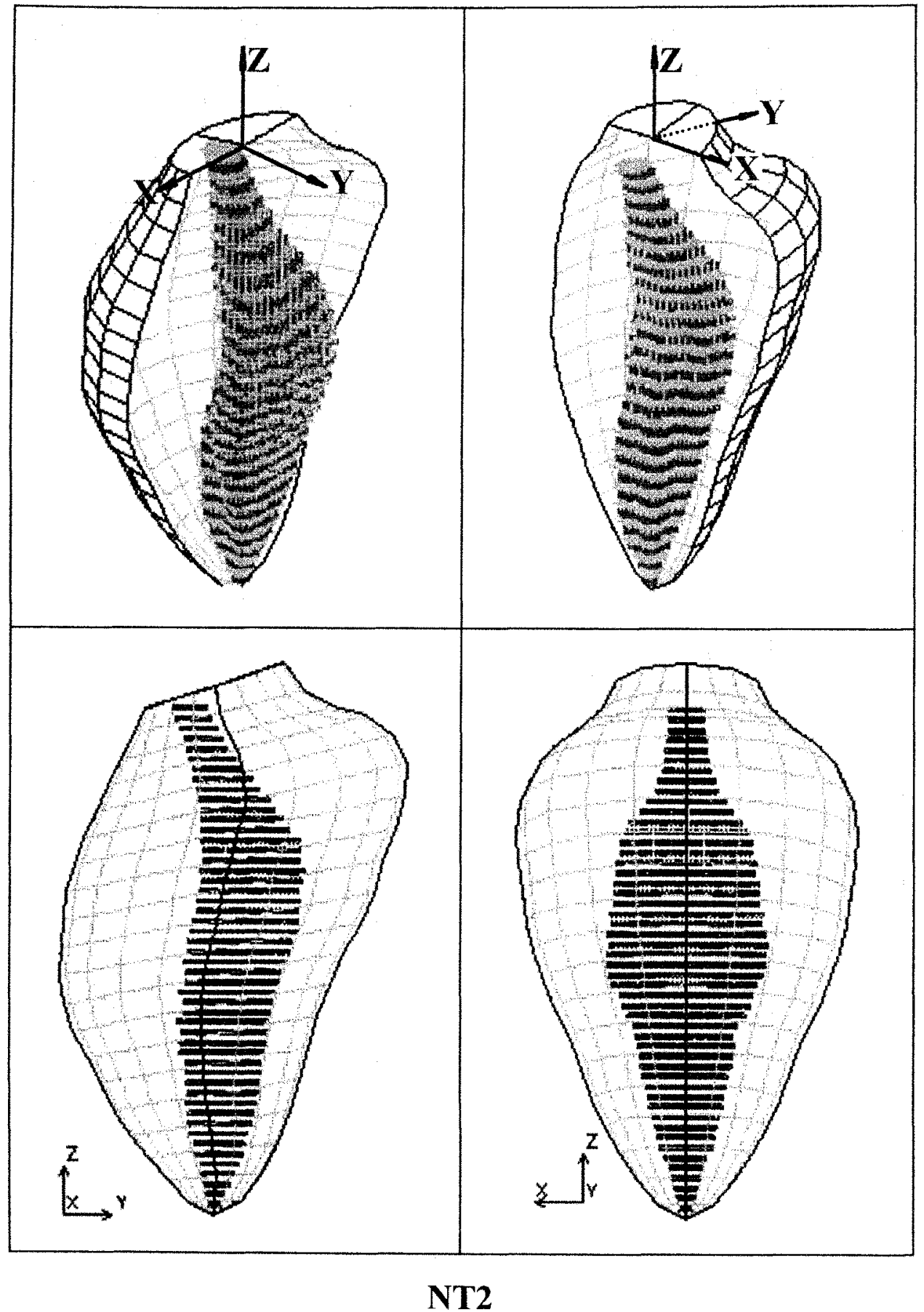

Figure4.6b Different views of the CER region inside the normal left ventricle $(\mathrm{NT} 2, \mathrm{t}=134 \mathrm{~ms})$ 


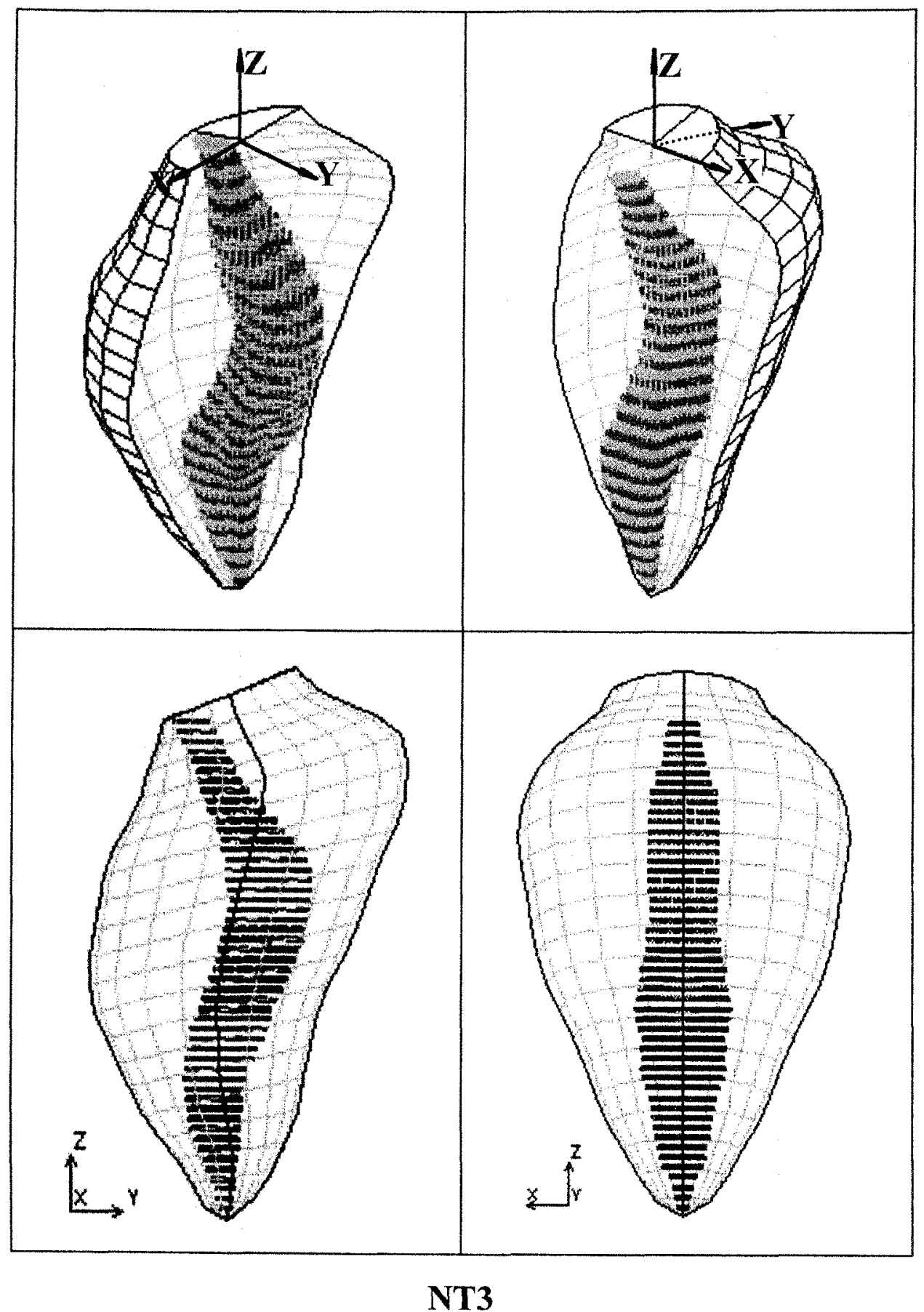

Figure4.6c Different views of the CER region inside the normal left ventricle (NT3, $t=201 \mathrm{~ms}$ ) 


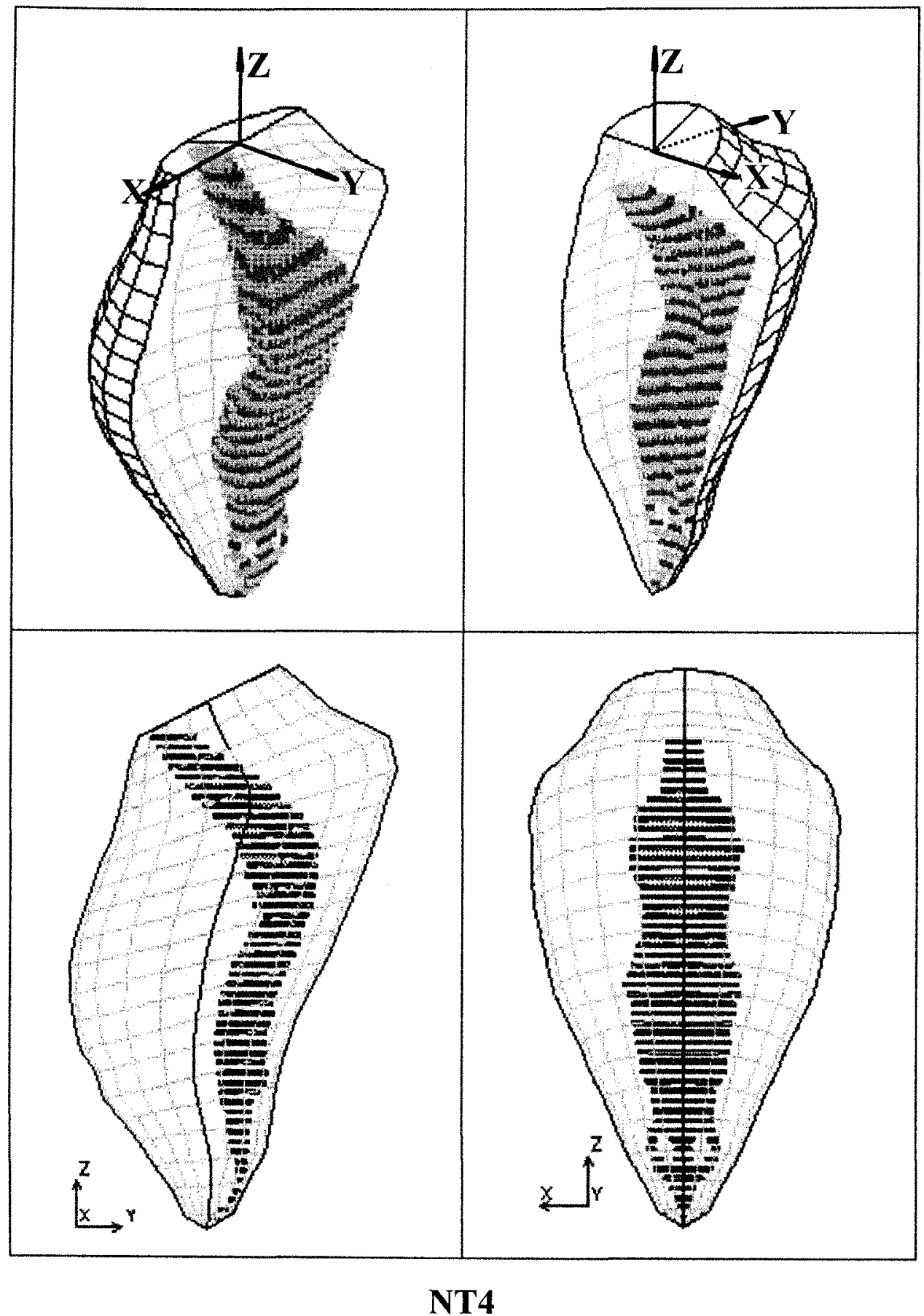

Figure4.6d Different views of the CER region inside the normal left ventricle (NT4, $t=301 \mathrm{~ms}$ ) 


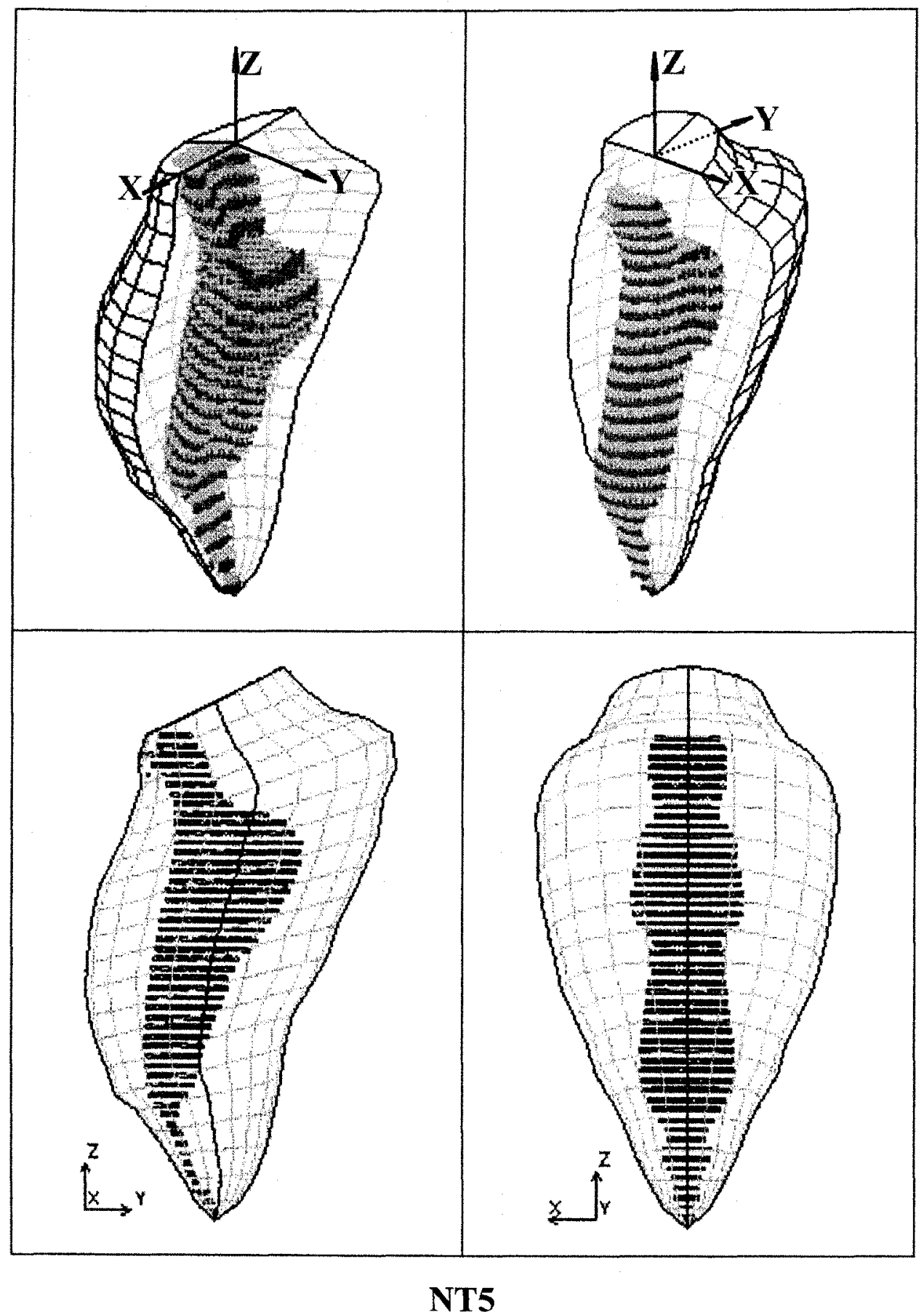

Figure4.6e Different views of the CER region inside the normal left ventricle (NT5, $t=401 \mathrm{~ms})$ 


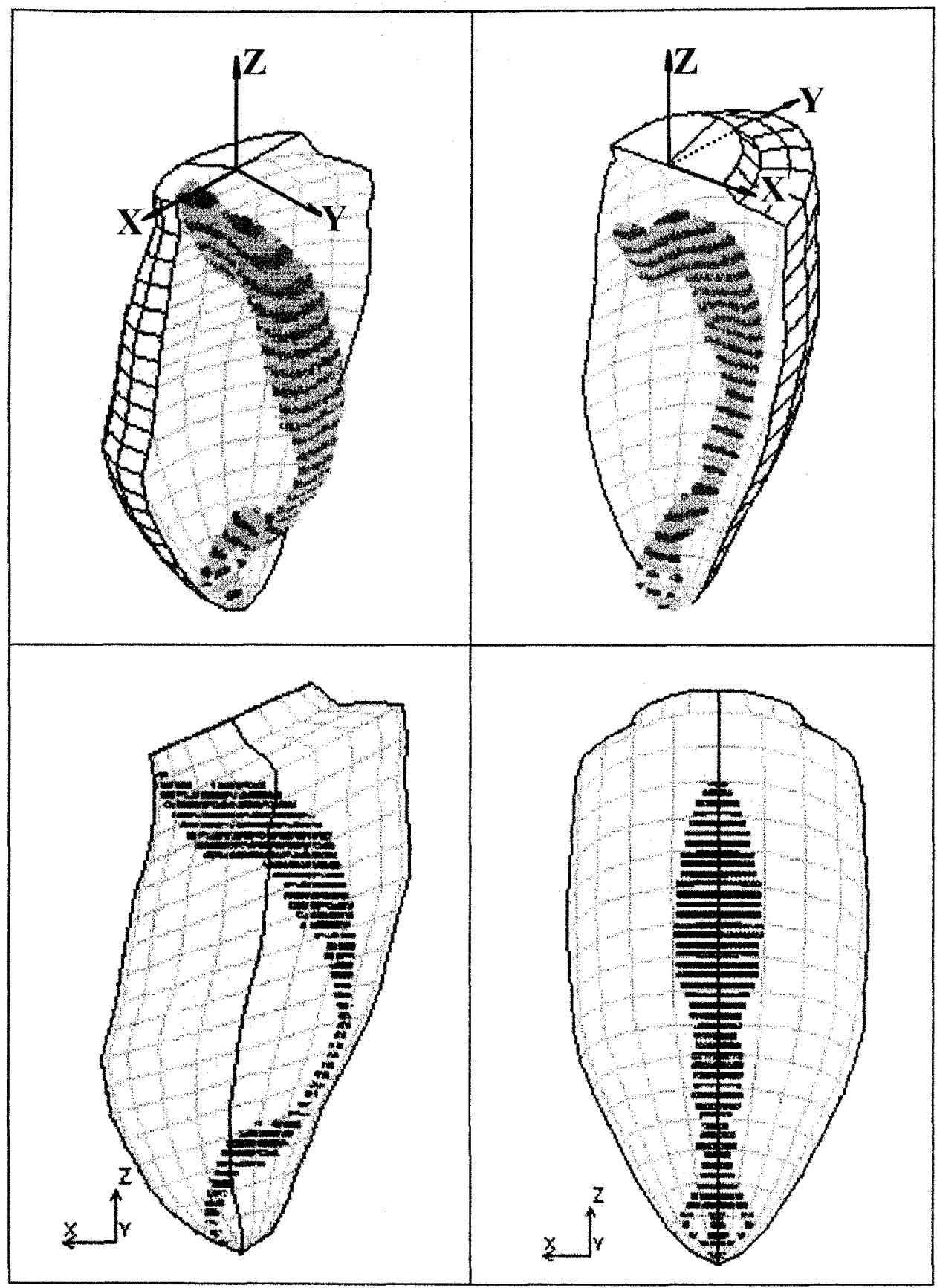

IT1

Figure4.7a Different views of the CER region inside the ischemic left ventricle (IT1, $t=100 \mathrm{~ms})$ 


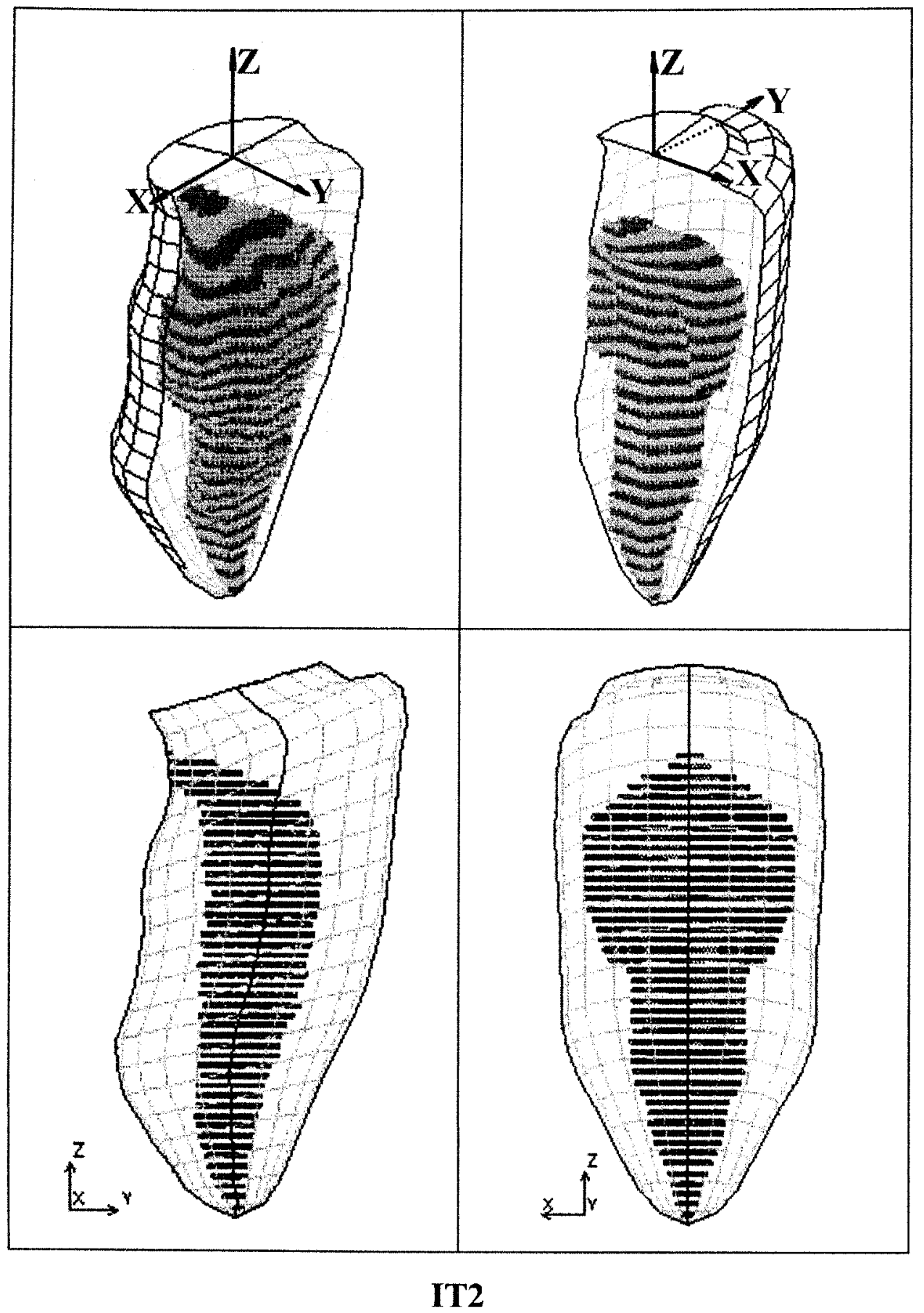

Figure4.7b Different views of the CER region inside the ischemic left ventricle (IT2, $t=200 \mathrm{~ms}$ ) 


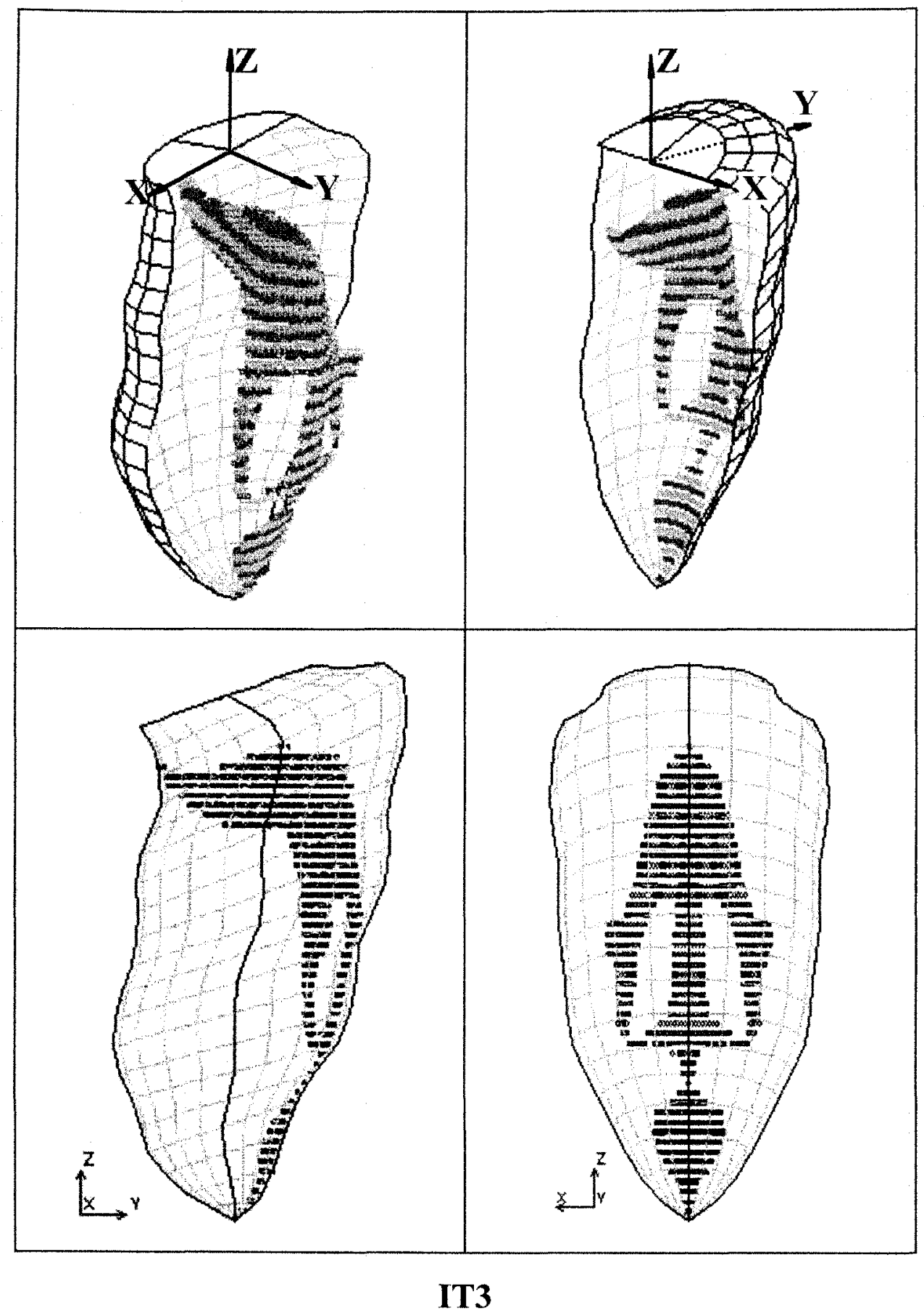

Figure4.7c Different views of the CER region inside the ischemic left ventricle (IT3, $t=267 \mathrm{~ms}$ ) 


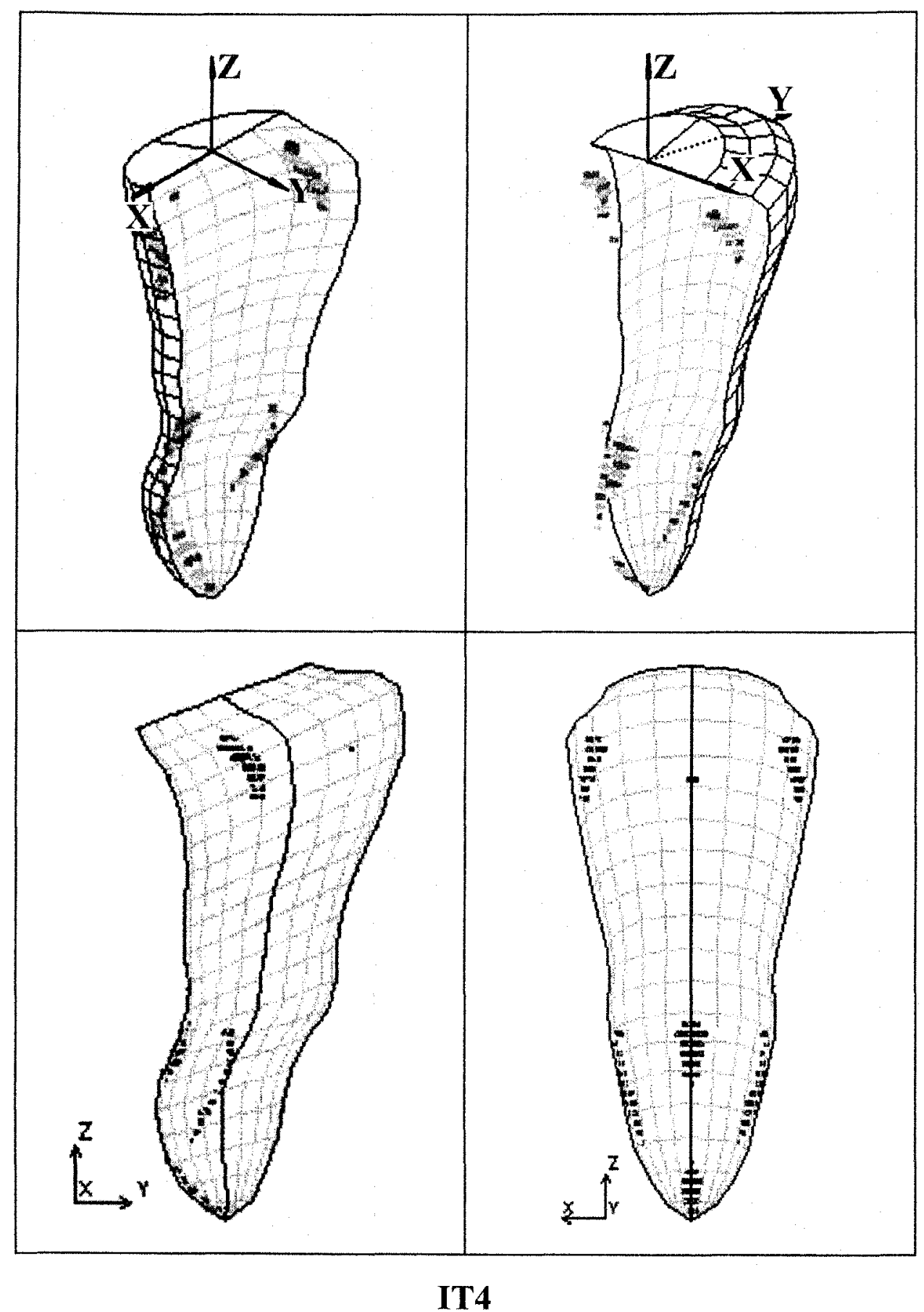

Figure 4.7d Different views of the CER region inside the Ischemic left ventricle (IT4, $t=334 \mathrm{~ms}$ ) 


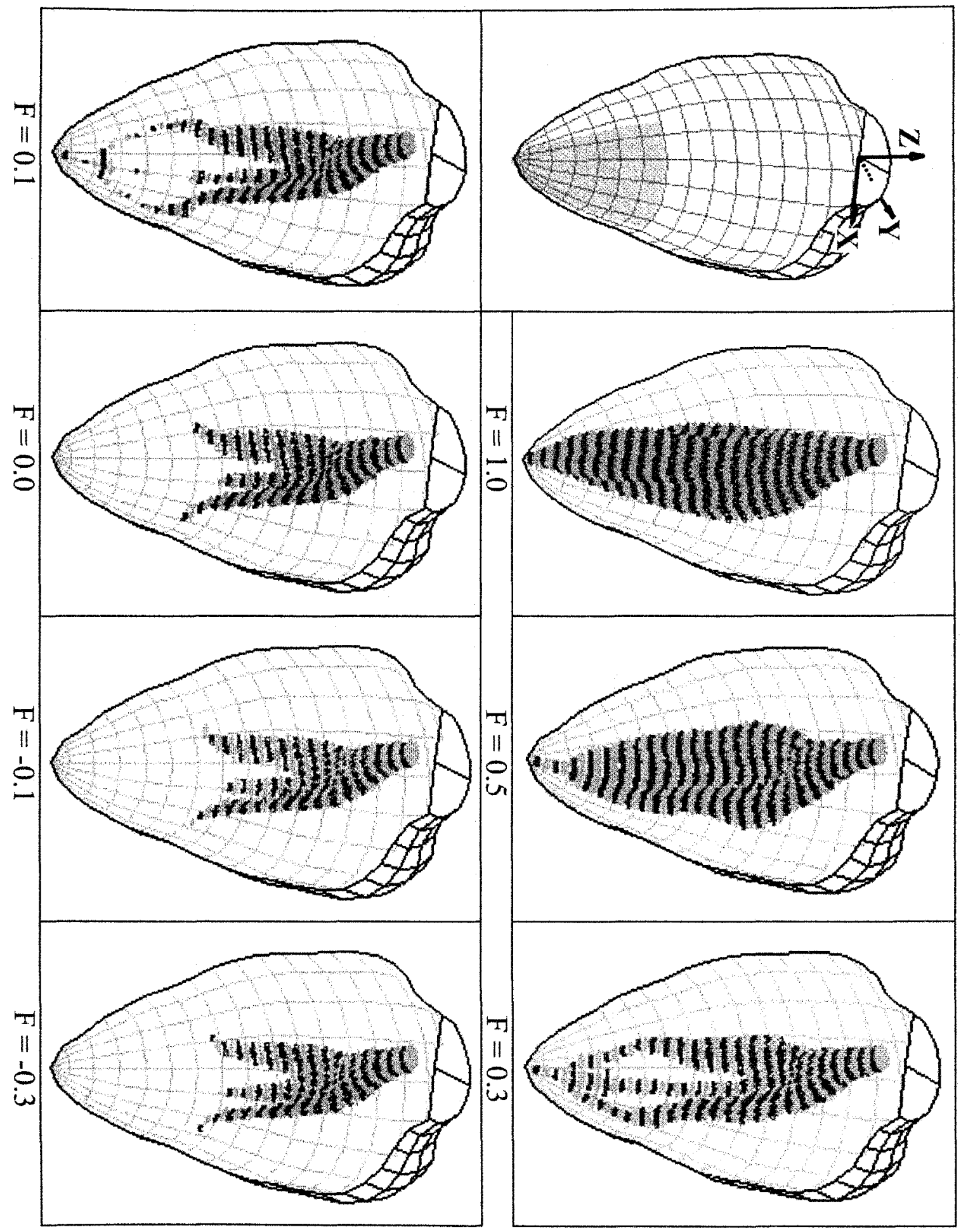

Figure4.8a 3D view of the CER regions inside left ventricles with simulated ischemic region small ischemia on the side 


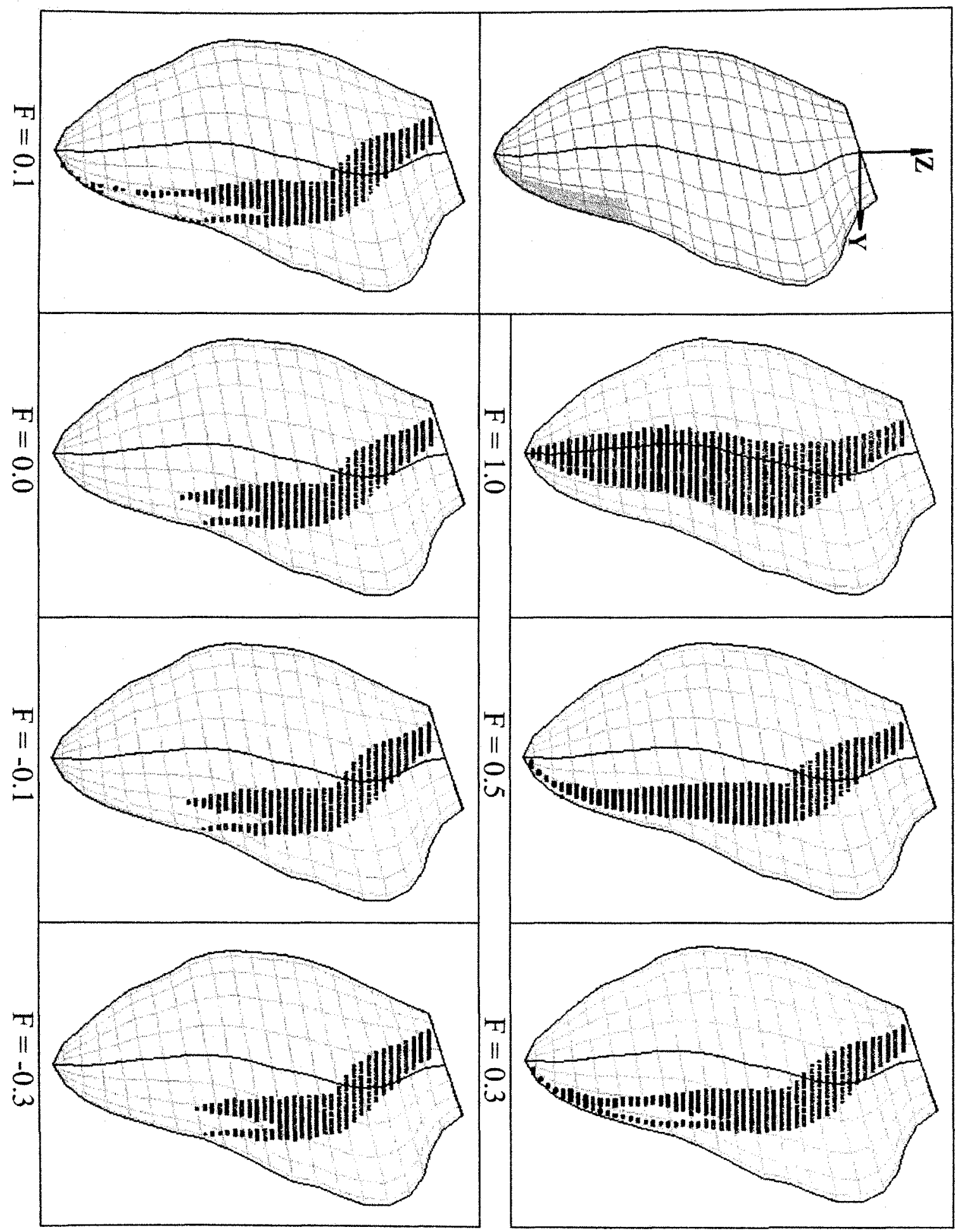

Figure $4.8 \mathrm{~b}$ Front view of the CER regions inside left ventricles with simulated ischemic region small ischemia on the side 


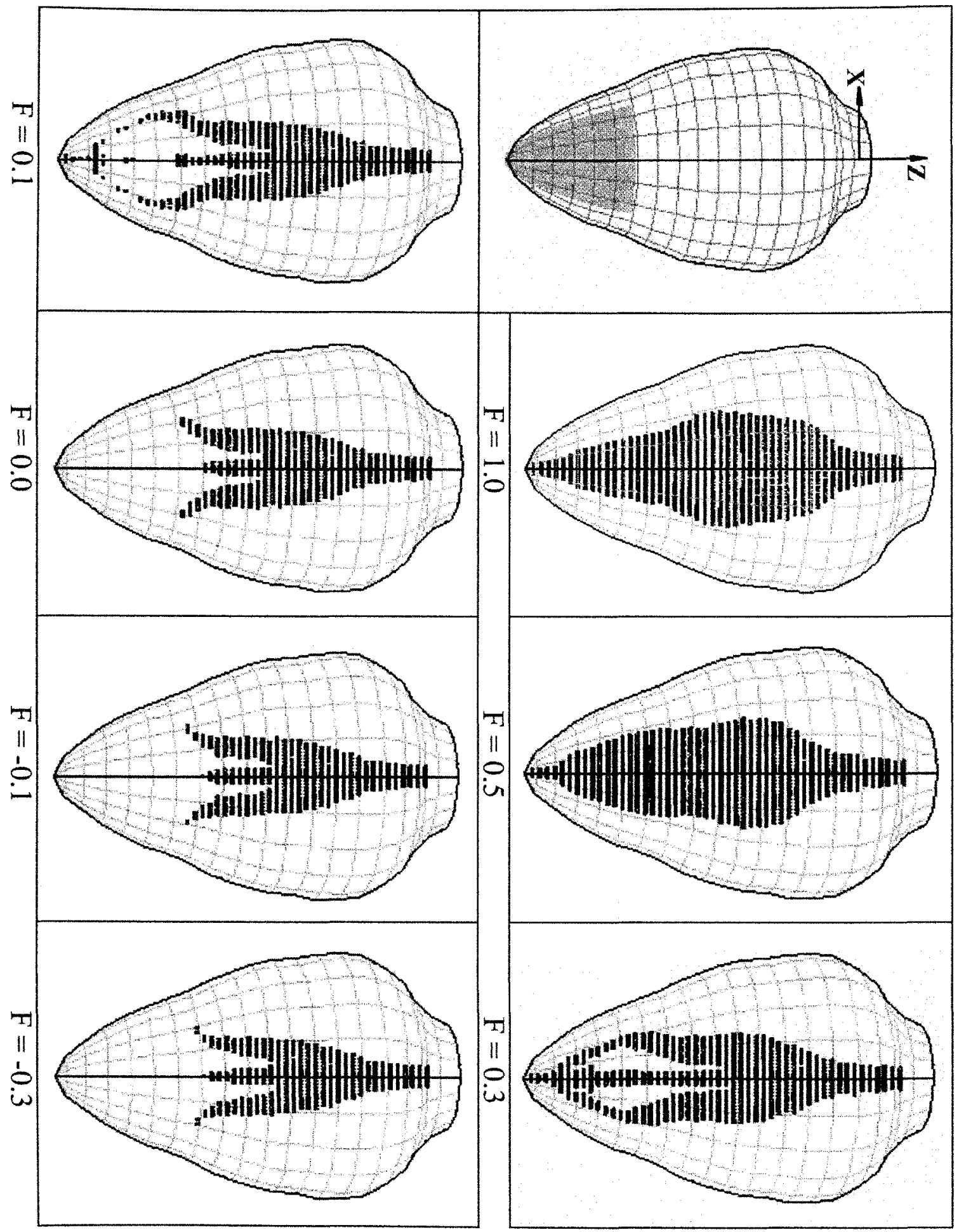

Figure $4.8 \mathrm{c}$ Side view of the CER regions inside left ventricles with simulated ischemic region small ischemia on the side 


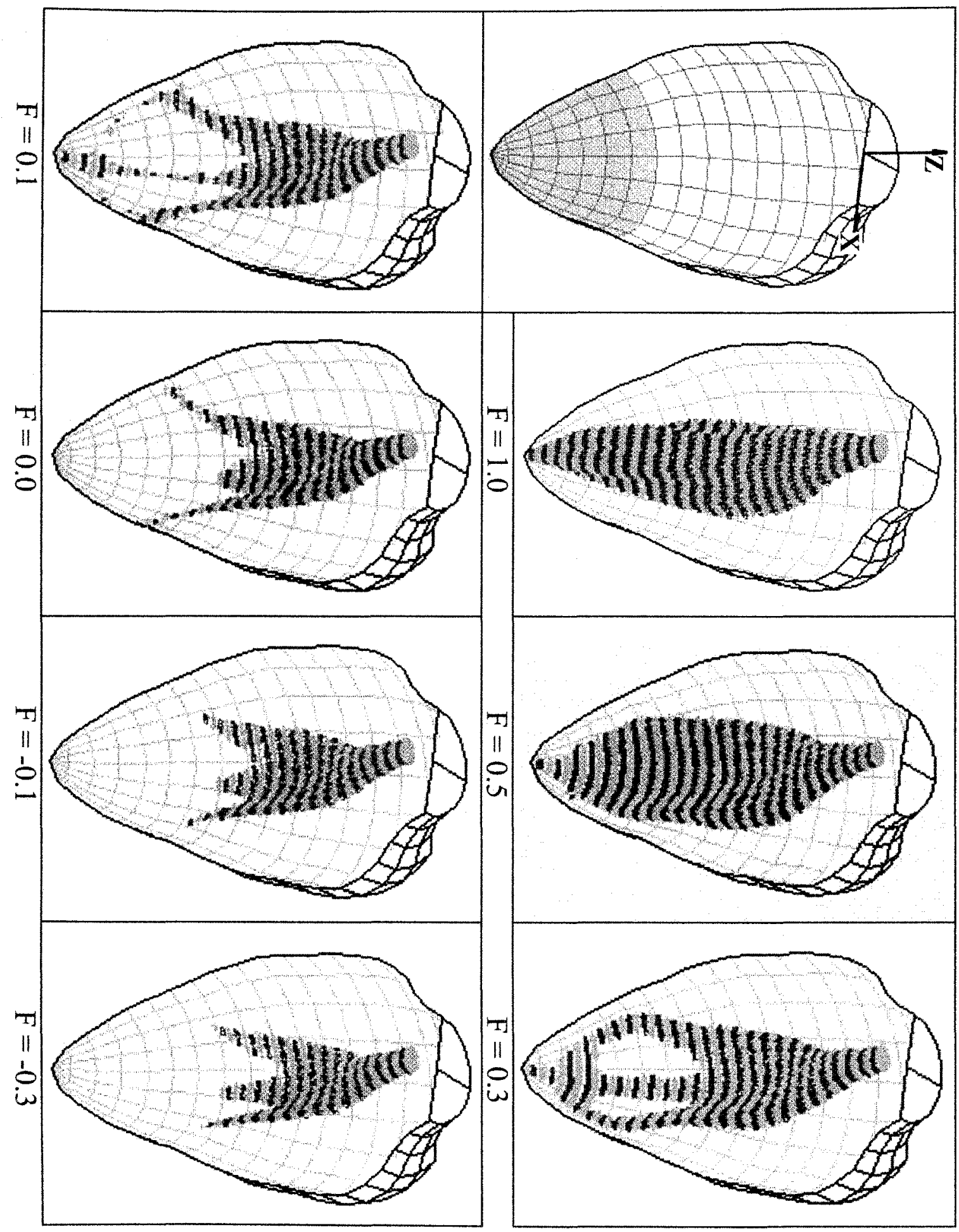

Figure 4.9a 3D view of the CER regions inside left ventricles with simulated ischemic region large ischemia on the side 


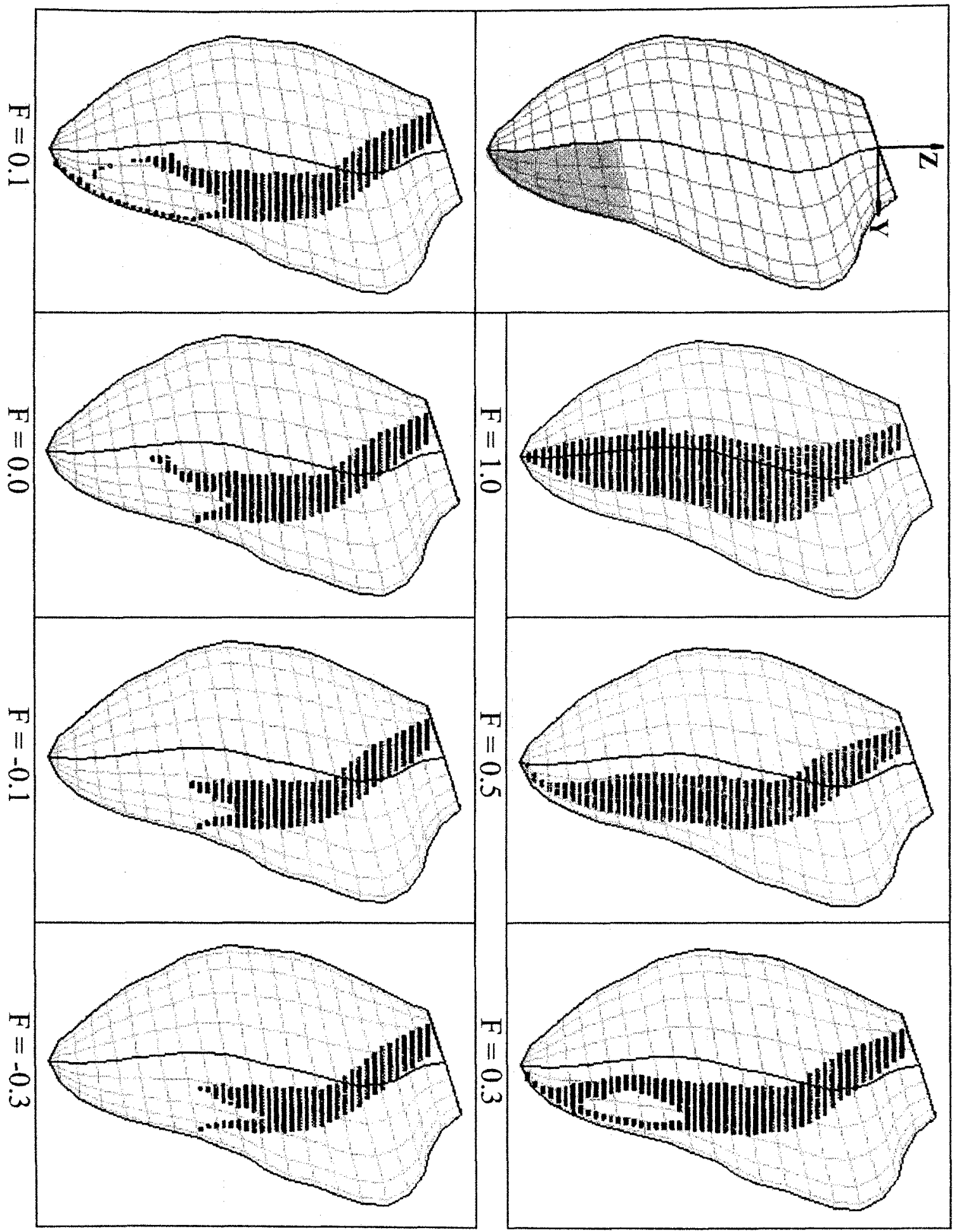

Figure 4.9b Front view of the CER regions inside left ventricles with simulated ischemic region large ischemia on the side 


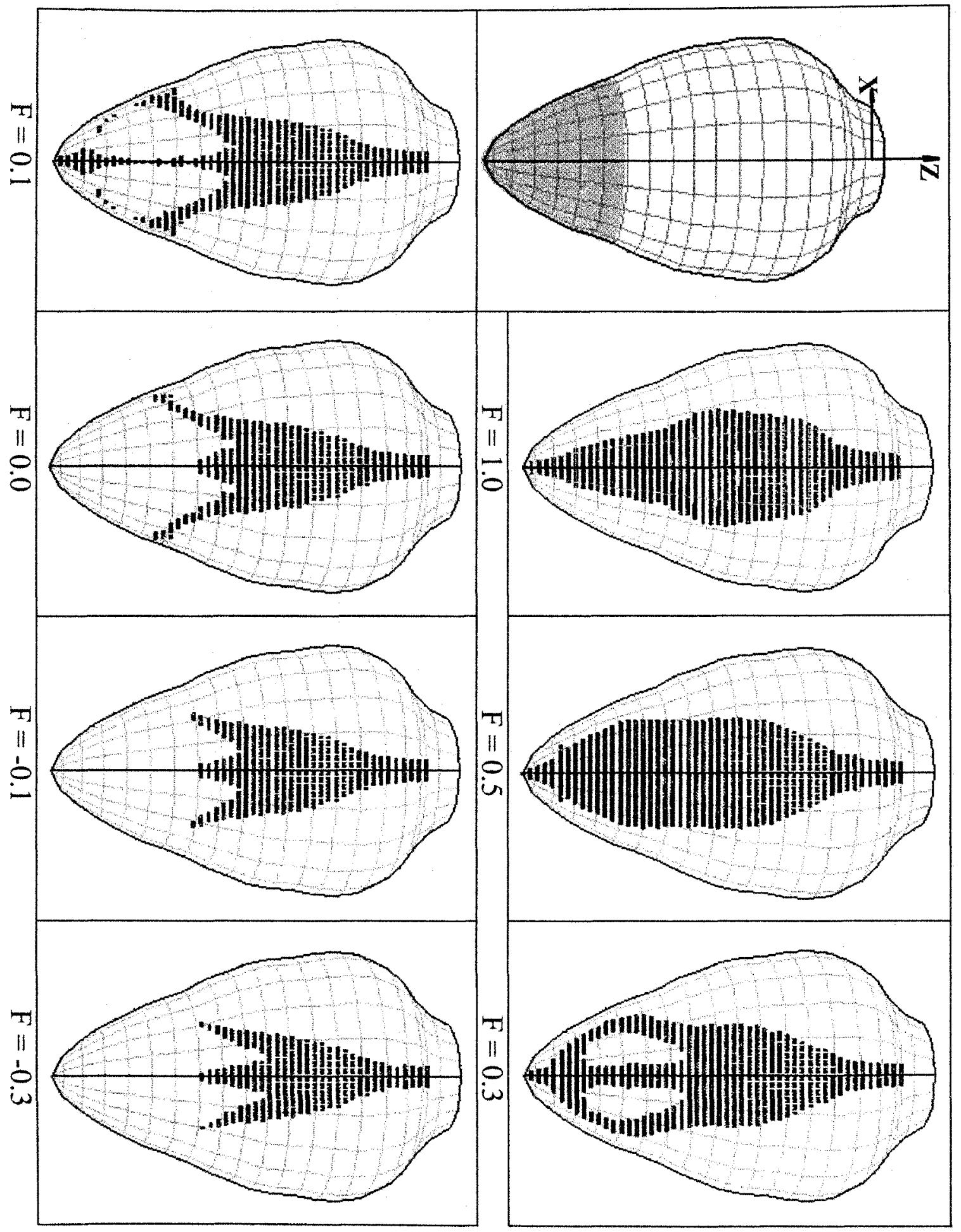

Figure $4.9 \mathrm{c}$ Side view of the CER regions inside left ventricles with simulated ischemic region large ischemia on the side 


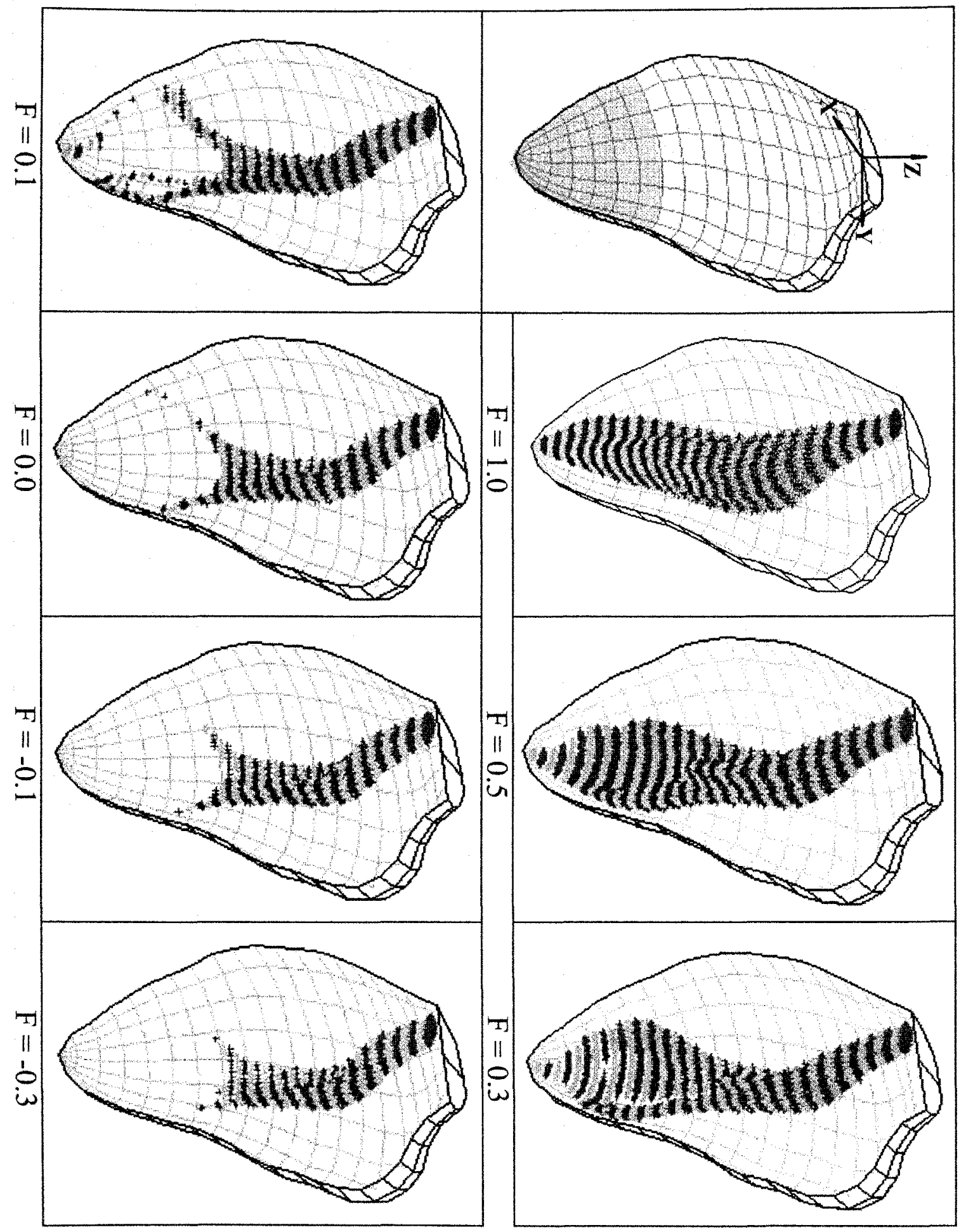

Figure 4.10a 3D view of the CER regions inside left ventricles with simulated ischemic region large ischemia at back 


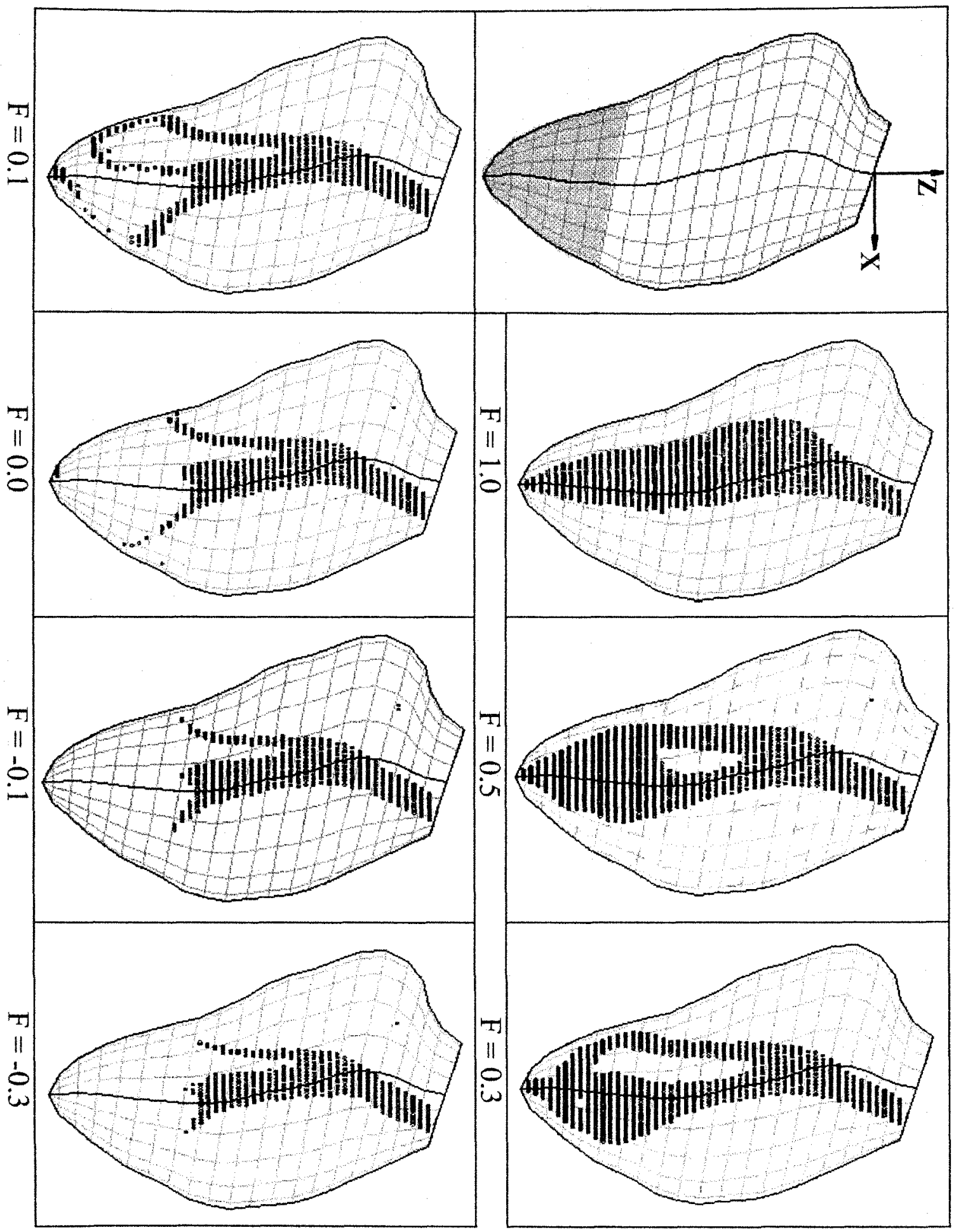

Figure 4.10b Back view of the CER regions inside left ventricles with simulated ischemic region large ischemia at back 


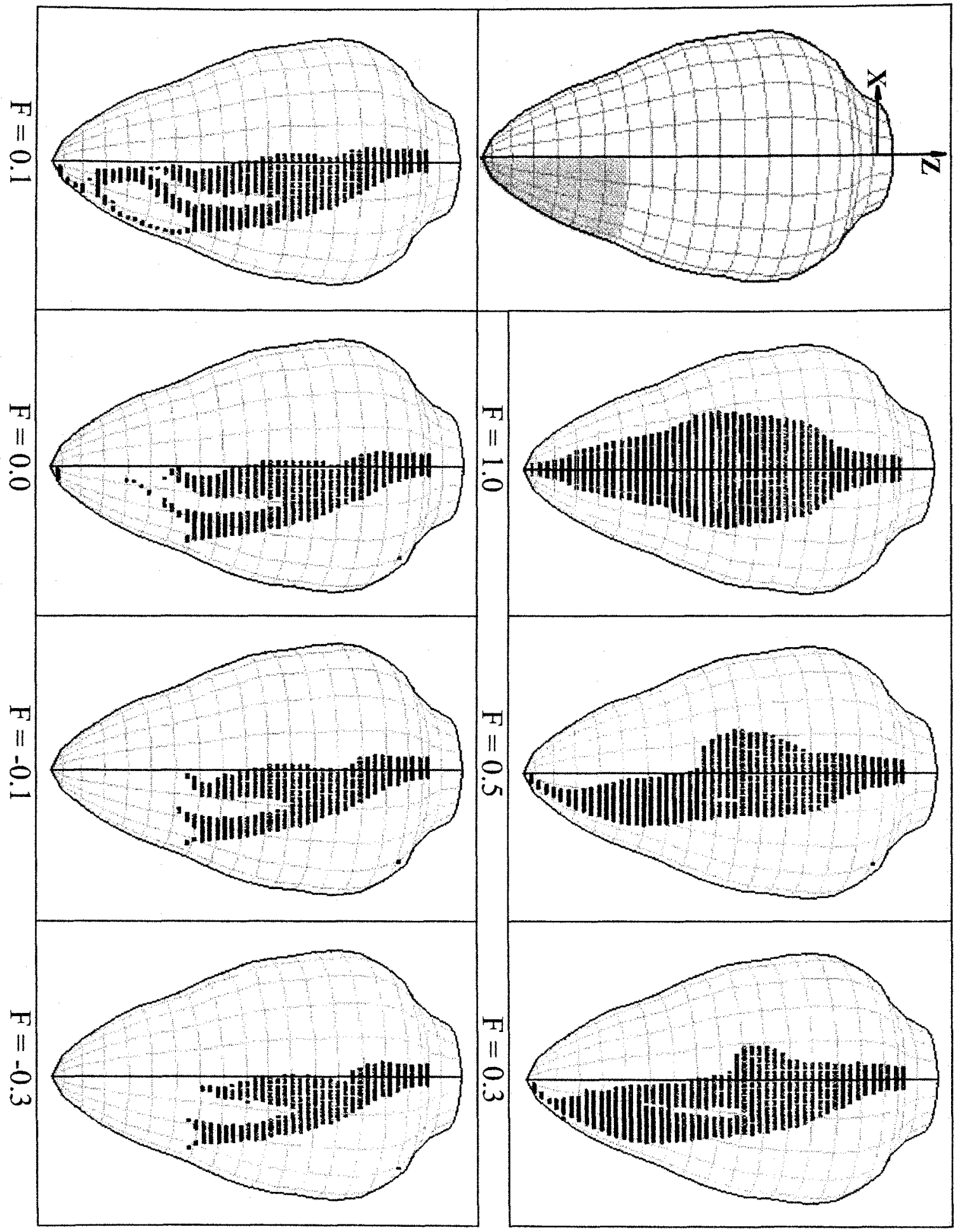

Figure4.10c Side view of the CER regions inside left ventricles with simulated ischemic region large ischemia at back 


\begin{tabular}{|c|c|c|c|c|}
\hline \multicolumn{5}{|c|}{ Normal Left Ventricle } \\
\hline $\begin{array}{c}\text { Time } \\
(\mathrm{ms})\end{array}$ & Time Step & $\begin{array}{c}\text { Outlet } \\
\text { diameter(cm) }\end{array}$ & $\begin{array}{c}\text { Outlet } \\
\text { velocity }(\mathrm{cm} / \mathrm{s})\end{array}$ & Re \\
\hline 0 & NT1 & 1.250 & 64.38 & $\mathbf{2 2 9 9 . 2 4}$ \\
67 & NT2 & 1.230 & 81.62 & $\mathbf{2 8 6 8 . 2 3}$ \\
201 & NT3 & 1.212 & 90.29 & $\mathbf{3 1 2 6 . 2 2}$ \\
301 & NT4 & 1.208 & 28.29 & $\mathbf{6 3 1 . 3 2}$ \\
401 & NT5 & 1.161 & 15.43 & $\mathbf{5 1 2 . 0 2}$ \\
\hline
\end{tabular}

Table 4.1 Time after onset of systole for each Normal LV outline and corresponding Re for each time step

\begin{tabular}{|c|c|c|c|c|}
\hline \multicolumn{5}{|c|}{ Ischemic Left Ventricle } \\
\hline $\begin{array}{c}\text { Time } \\
(\mathrm{ms})\end{array}$ & Time Step & $\begin{array}{c}\text { Outlet } \\
\text { diameter(cm) }\end{array}$ & $\begin{array}{c}\text { Outlet } \\
\text { velocity }(\mathrm{cm} / \mathrm{s})\end{array}$ & Re \\
\hline 0 & IT1 & 1.250 & 27.45 & $\mathbf{9 8 0 . 3 8}$ \\
100 & IT2 & 1.24 & 34.23 & $\mathbf{1 2 0 9 . 0 9}$ \\
200 & IT3 & 1.16 & 39.76 & $\mathbf{1 3 1 7 . 6 9}$ \\
334 & IT4 & 1.13 & 7.12 & $\mathbf{2 2 9 . 7 5 7}$ \\
\hline
\end{tabular}

Table 4.2 Time after onset of systole for each Ischemic LV outline and corresponding $\mathrm{Re}$ for each time step 


\begin{tabular}{|c|ccccc|c|}
\hline \multicolumn{2}{|c}{ NT1 } & NT2 & NT3 & NT4 & NT5 & $\begin{array}{c}\text { Time } \\
\text { Ave. }\end{array}$ \\
\hline \multirow{2}{*}{ Normal } & 0.560 & 0.832 & 0.770 & 0.514 & 0.676 & 0.658 \\
\hline & IT1 & IT2 & IT3 & IT4 & Time Ave. \\
\hline \multirow{2}{*}{ Ischemic } & 0.448 & 0.783 & 0.329 & 0.054 & 0.362 \\
\hline
\end{tabular}

Table 4.3 CER coefficients of each time step of

Normal and Ischemic left ventricle

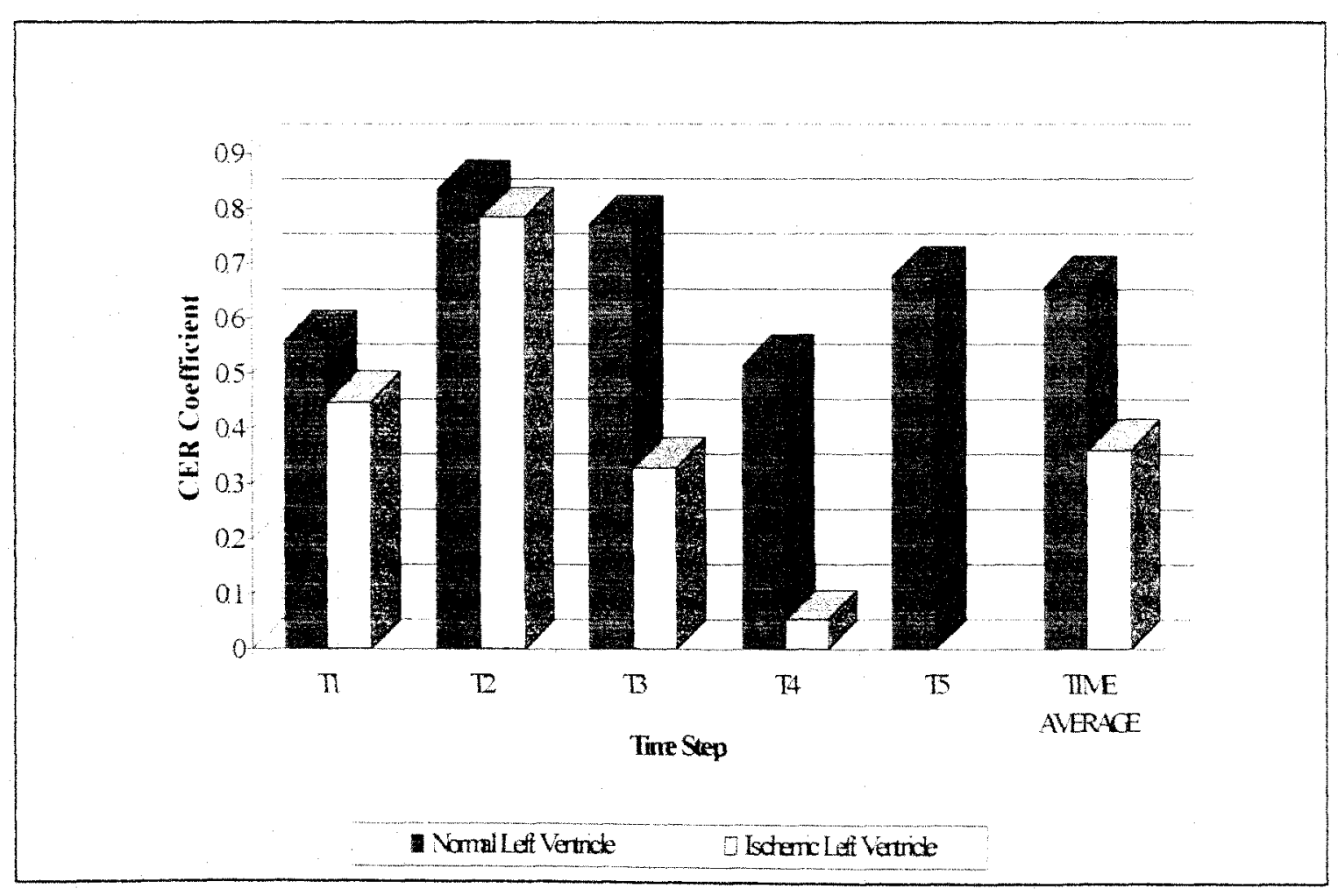

Figure 4.11 Cer coefficient vs. Time Steps for normal and ischemic left ventricles 


\begin{tabular}{|c|cccccc|}
\hline & $\mathbf{F}=\mathbf{0 . 5}$ & $\mathbf{F}=\mathbf{0 . 3}$ & $\mathbf{F}=\mathbf{0 . 1}$ & $\mathbf{F}=\mathbf{0 . 0}$ & $\mathbf{F}=\mathbf{0 . 1}$ & $\mathbf{F}=\mathbf{0 . 3}$ \\
\hline $\begin{array}{c}\text { Size } \\
\text { small @ } \\
\text { side }\end{array}$ & 0.533 & 0.466 & 0.420 & 0.394 & 0.387 & 0.376 \\
\hline $\begin{array}{c}\text { Size } \\
\text { Large @ } \\
\text { side }\end{array}$ & 0.588 & 0.511 & 0.456 & 0.424 & 0.407 & 0.399 \\
\hline $\begin{array}{c}\text { Size } \\
\text { Large @ } \\
\text { back }\end{array}$ & 0.634 & 0.553 & 0.469 & 0.451 & 0.415 & 0.406 \\
\hline
\end{tabular}

Table 4.4 CER coefficient of simulated iscemic left ventricles

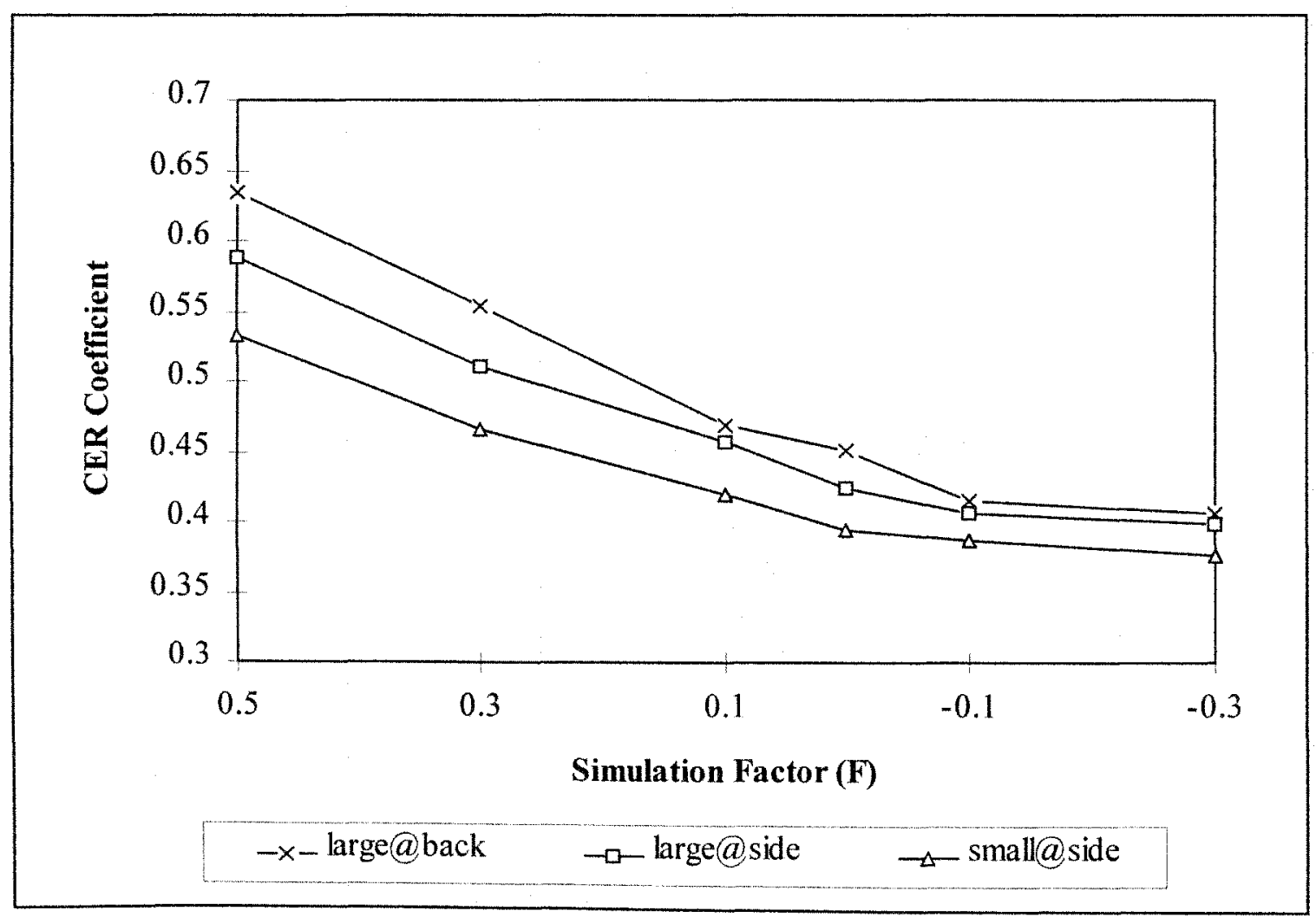

Figure 4.12 CER coefficient vs. simulation factor 


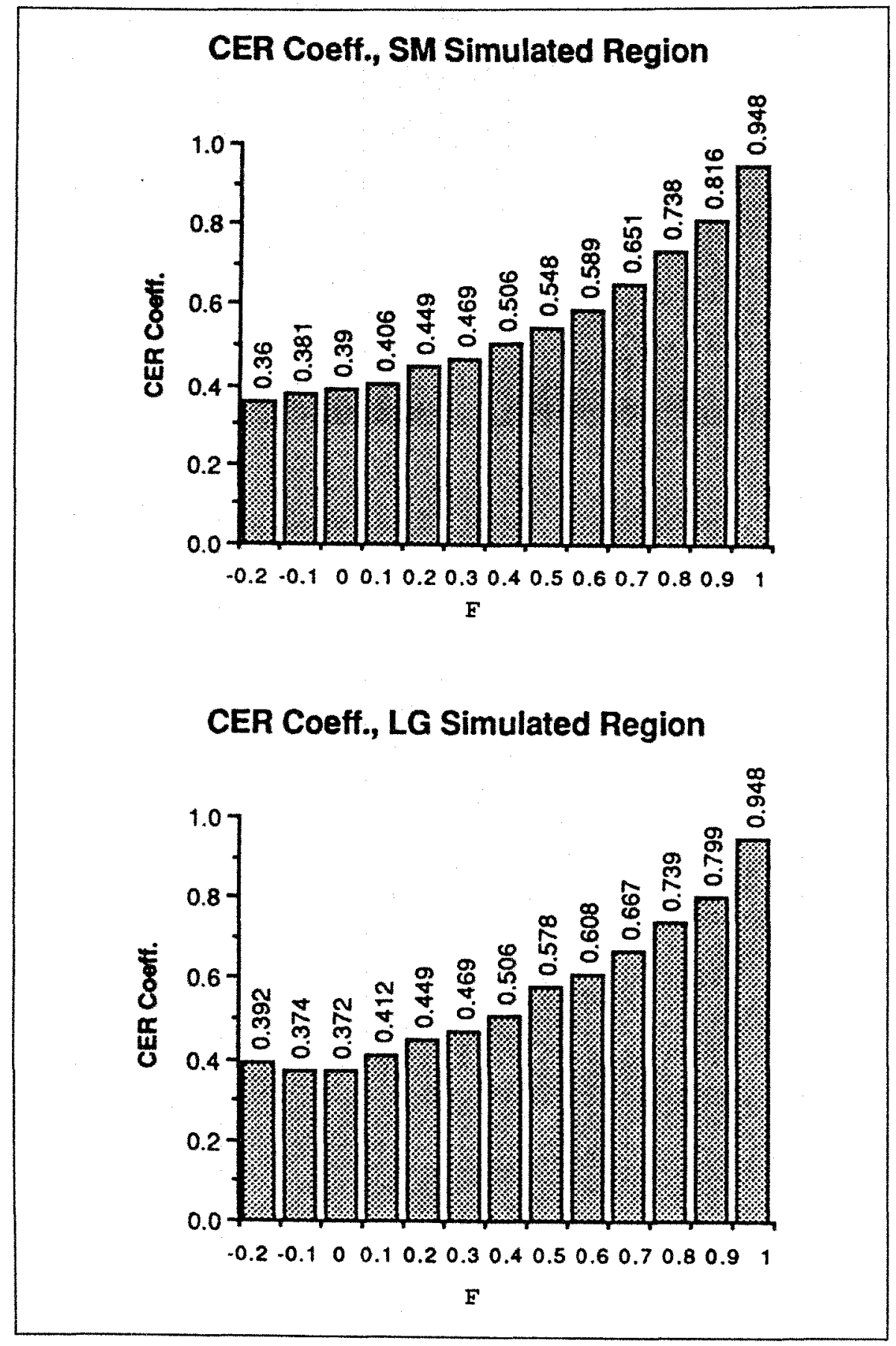

Figure 4.13 CER coefficient vs. Simulation Factor for sphere shape model 


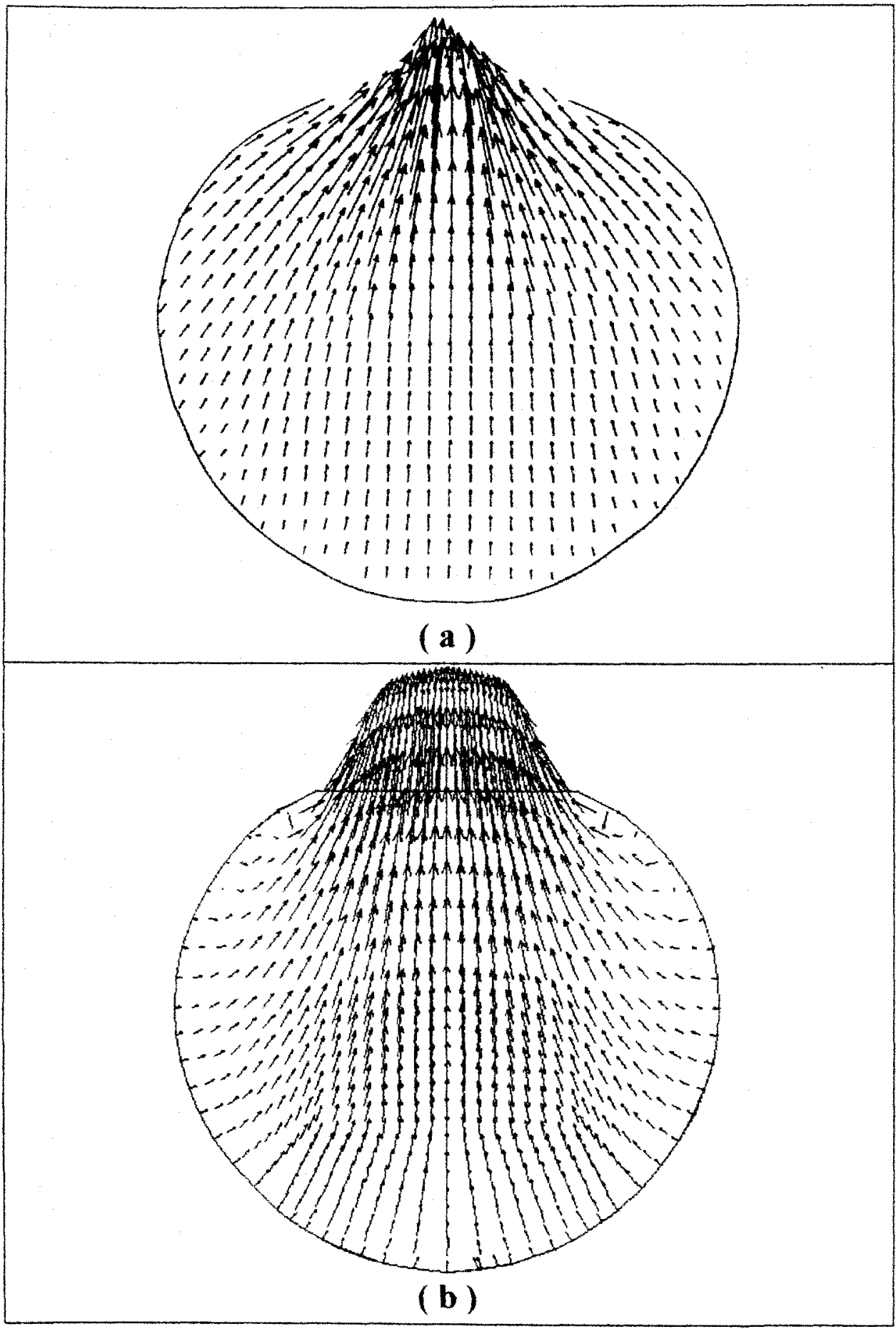

Figure4.14 Velocity vector plots from the numerical simulation and experimental measurements for normal left ventricle (a) Experimental measurement (b) Numerical simulation 


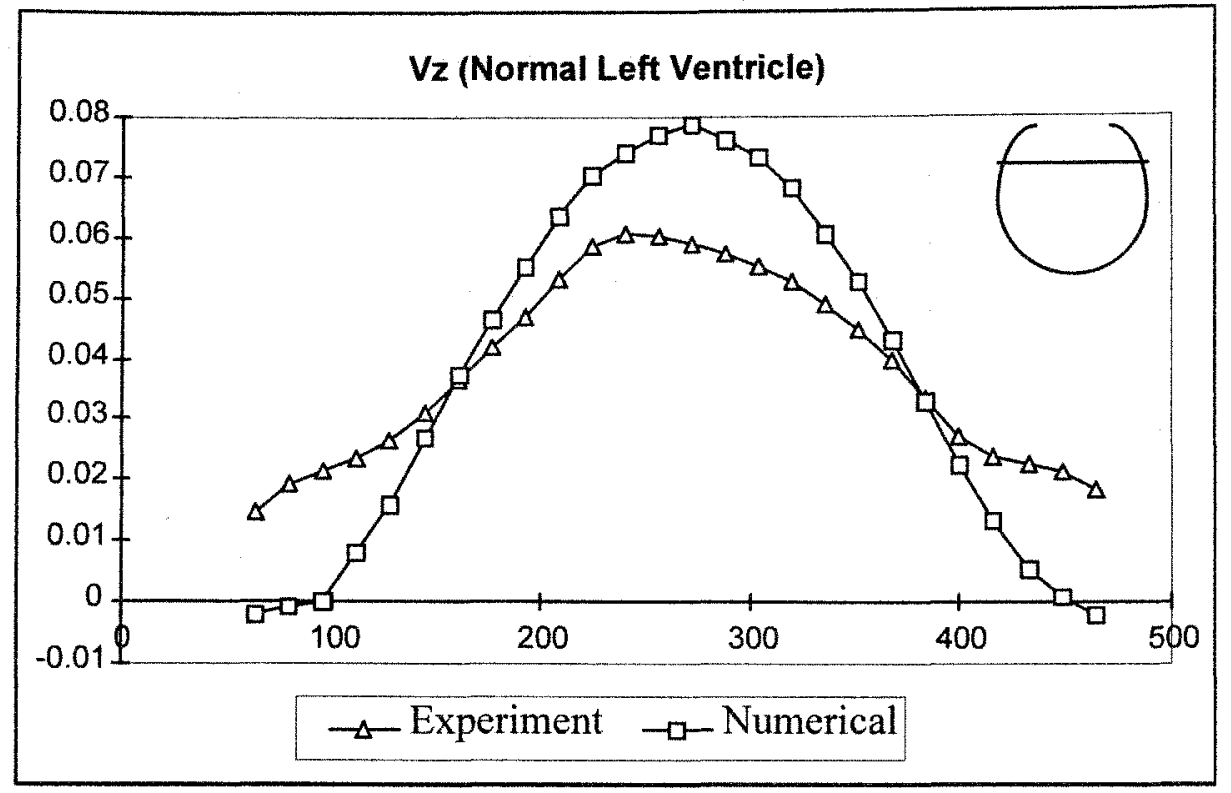

(a)

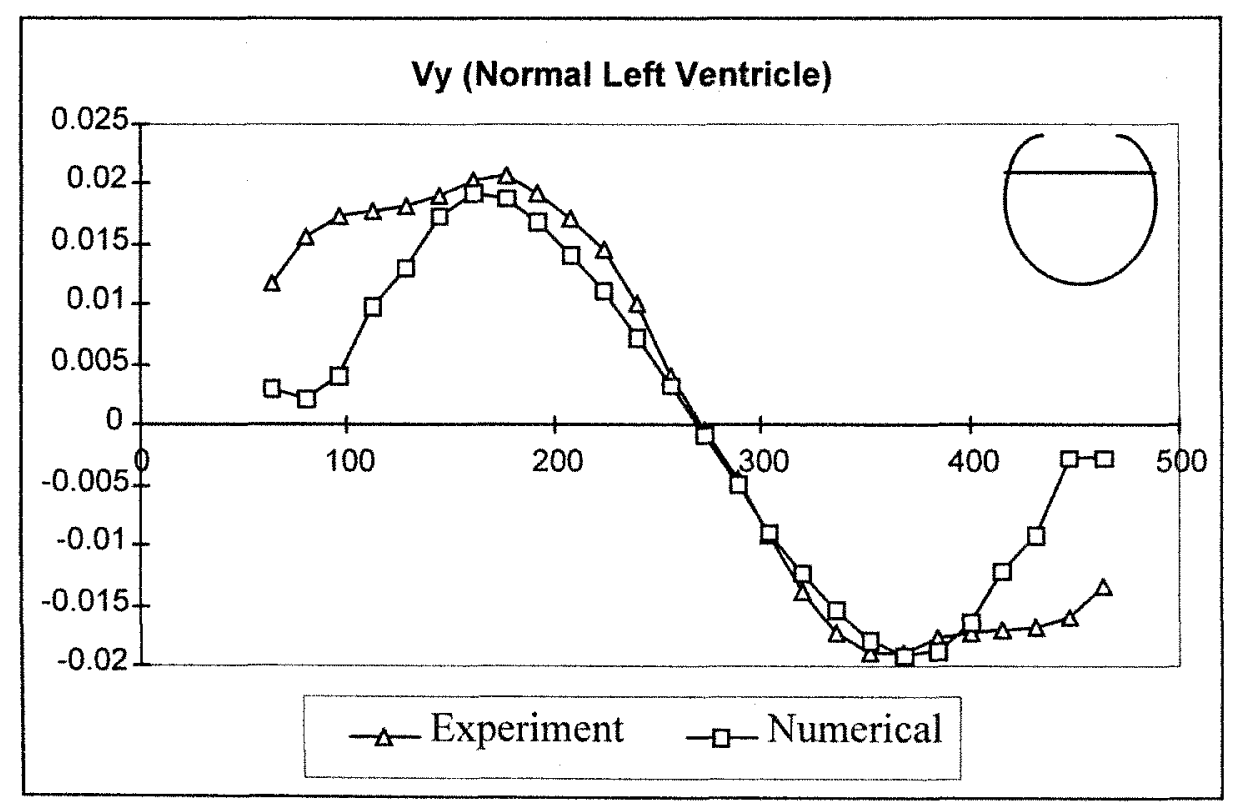

(b)

Figure 4.15 Velocity profile of experimental and numerical results close to outlet
(a) $\mathrm{Z}$ component
(b) Y component 


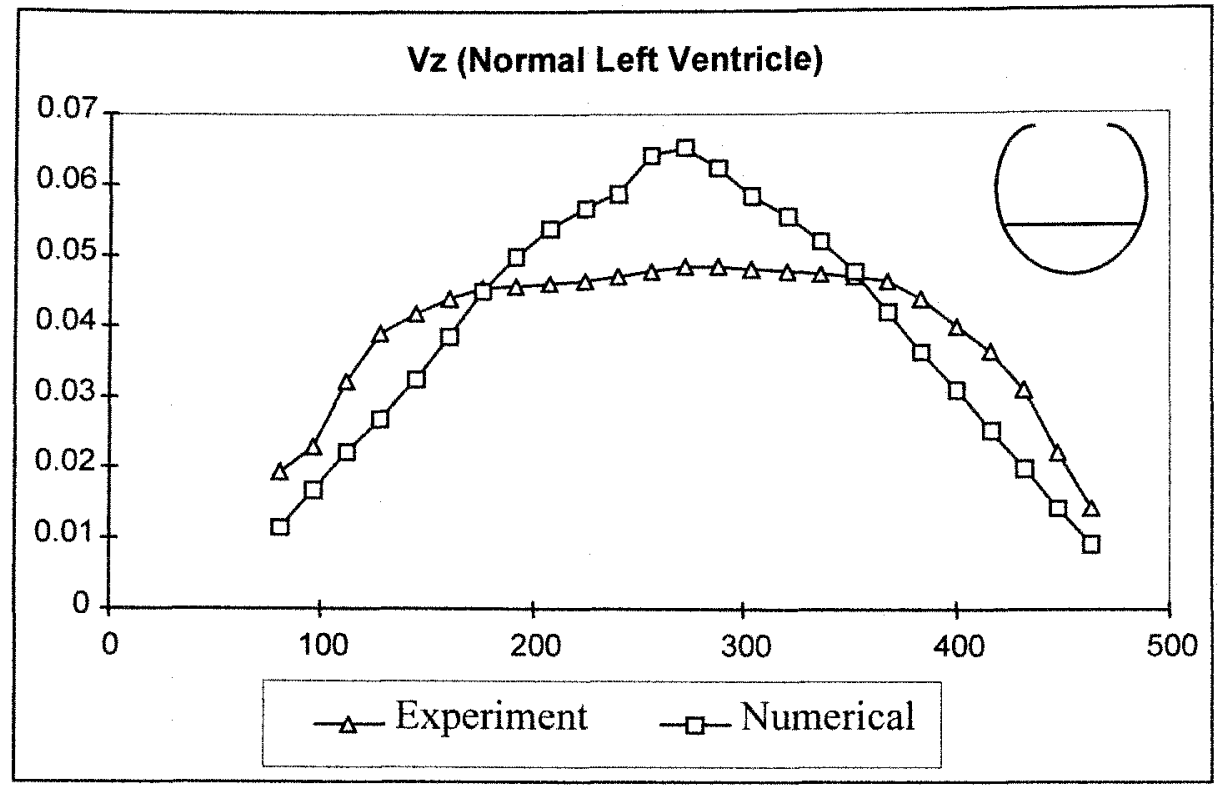

(a)

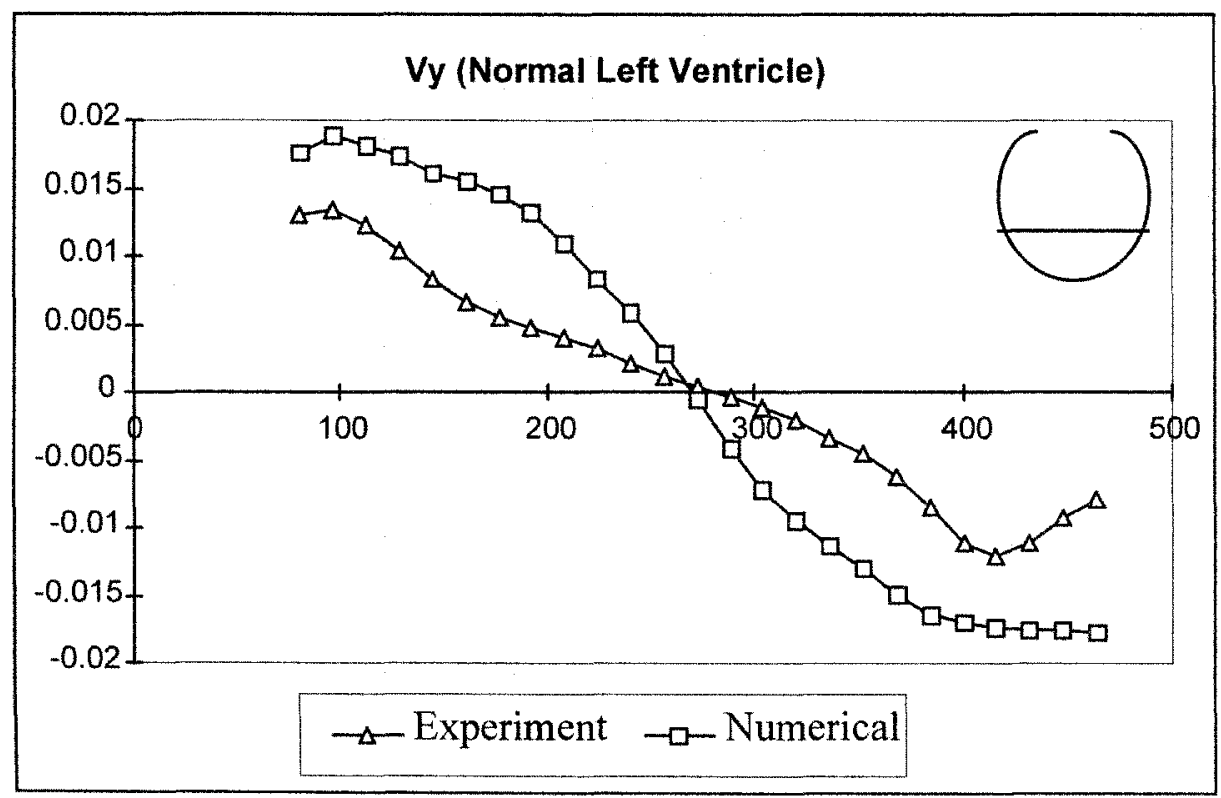

(b)

Figure 4.16 Velocity profile of experimental and numerical results close to bottom
(a) $\mathrm{Z}$ component
(b) Y component 


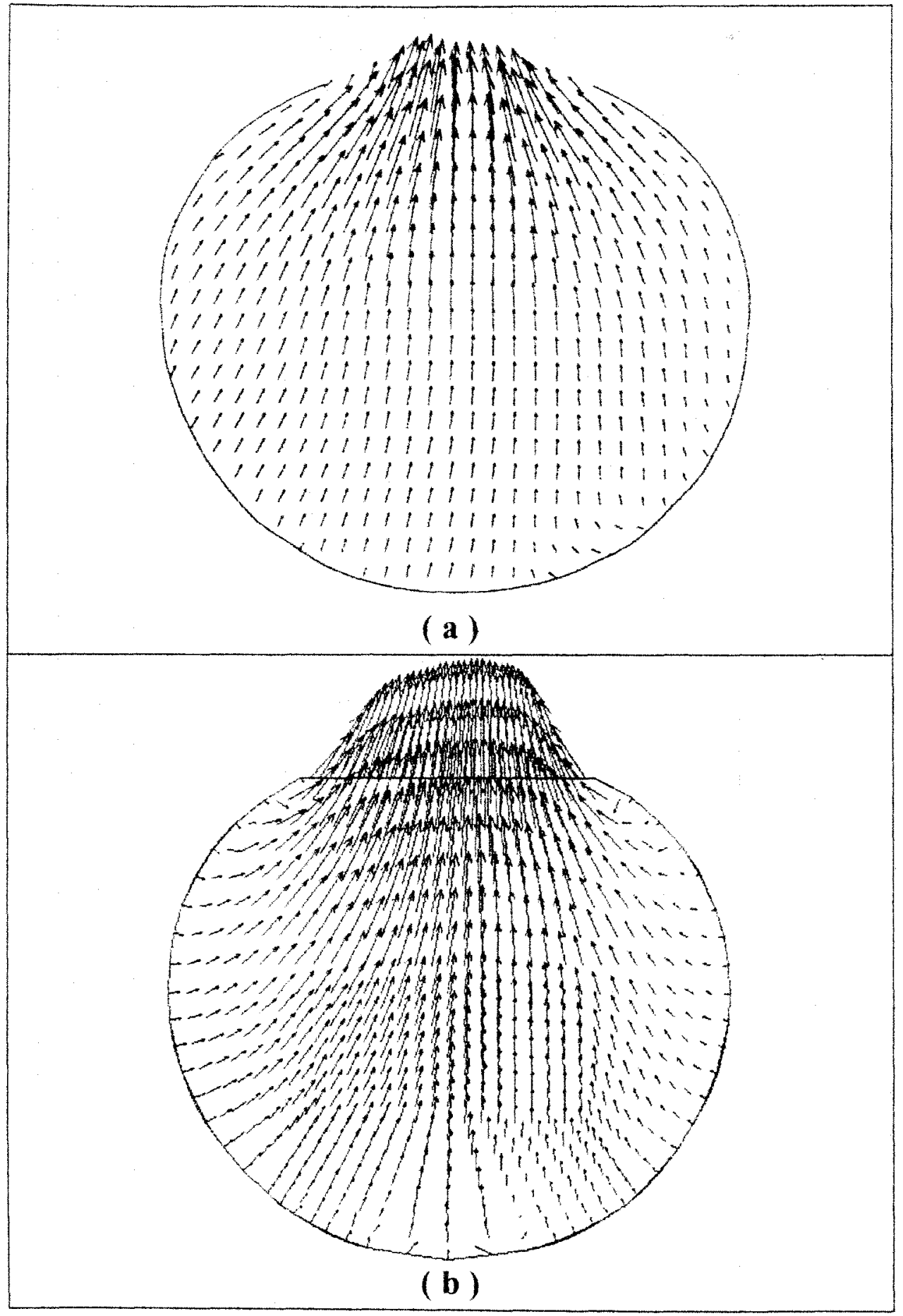

Figure4.17 Velocity vector plots from the numerical simulation and experimental measurements for ischemic left ventricle (a) Experimental measurement (b) Numerical simulation 


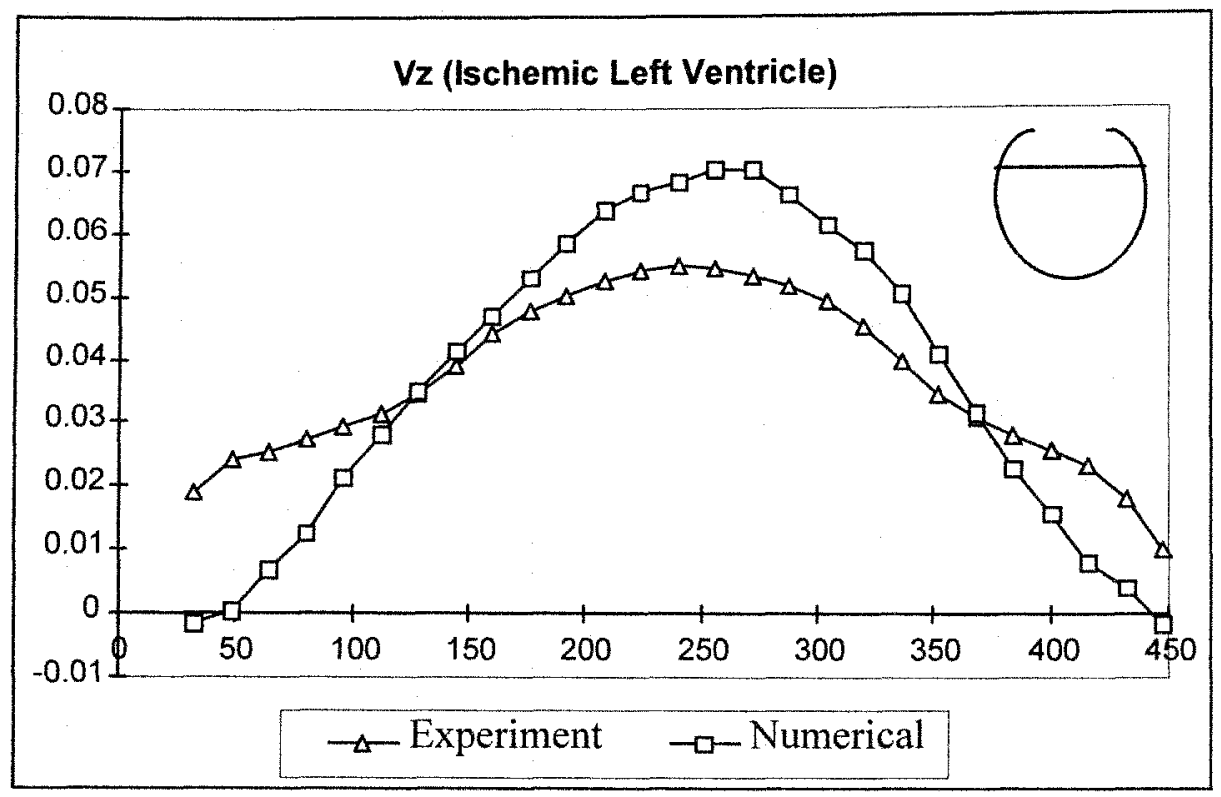

(a)

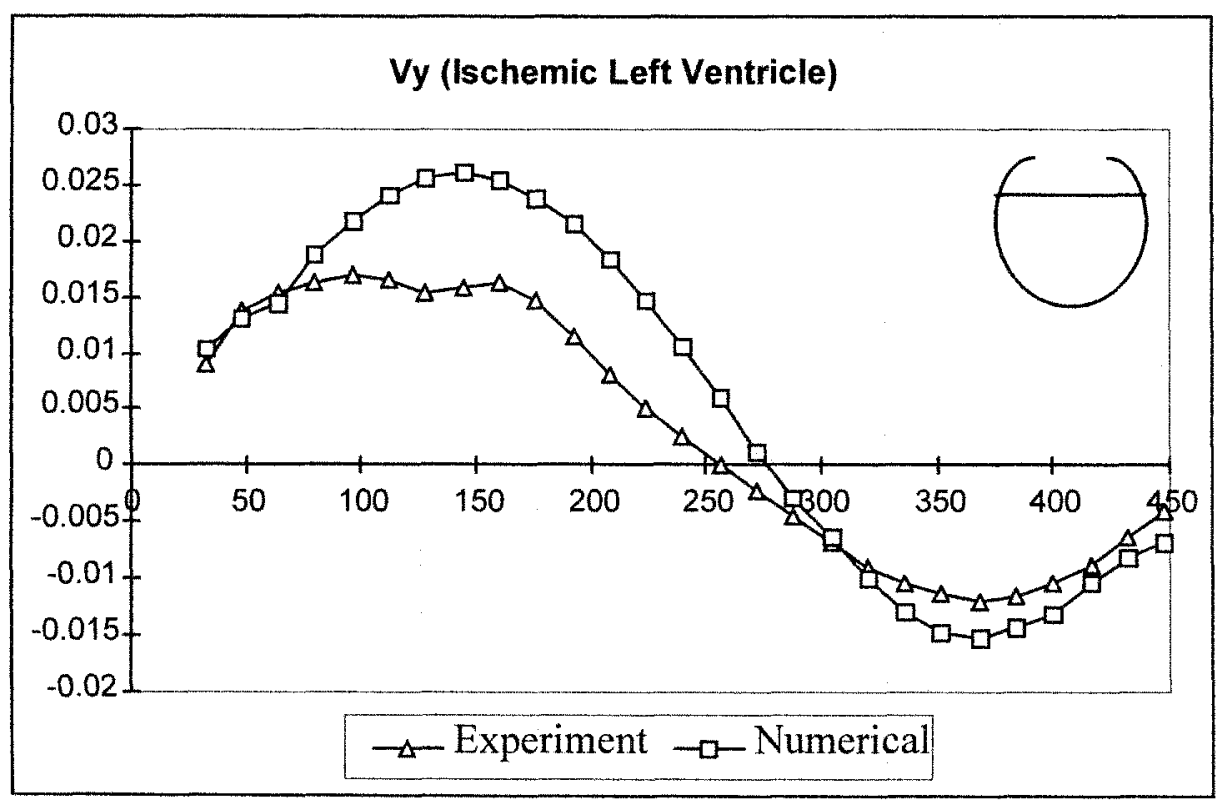

(b)

Figure 4.18 Velocity profile of experimental and numerical results close to bottom
(a) $\mathrm{Z}$ component
(b) Y component 


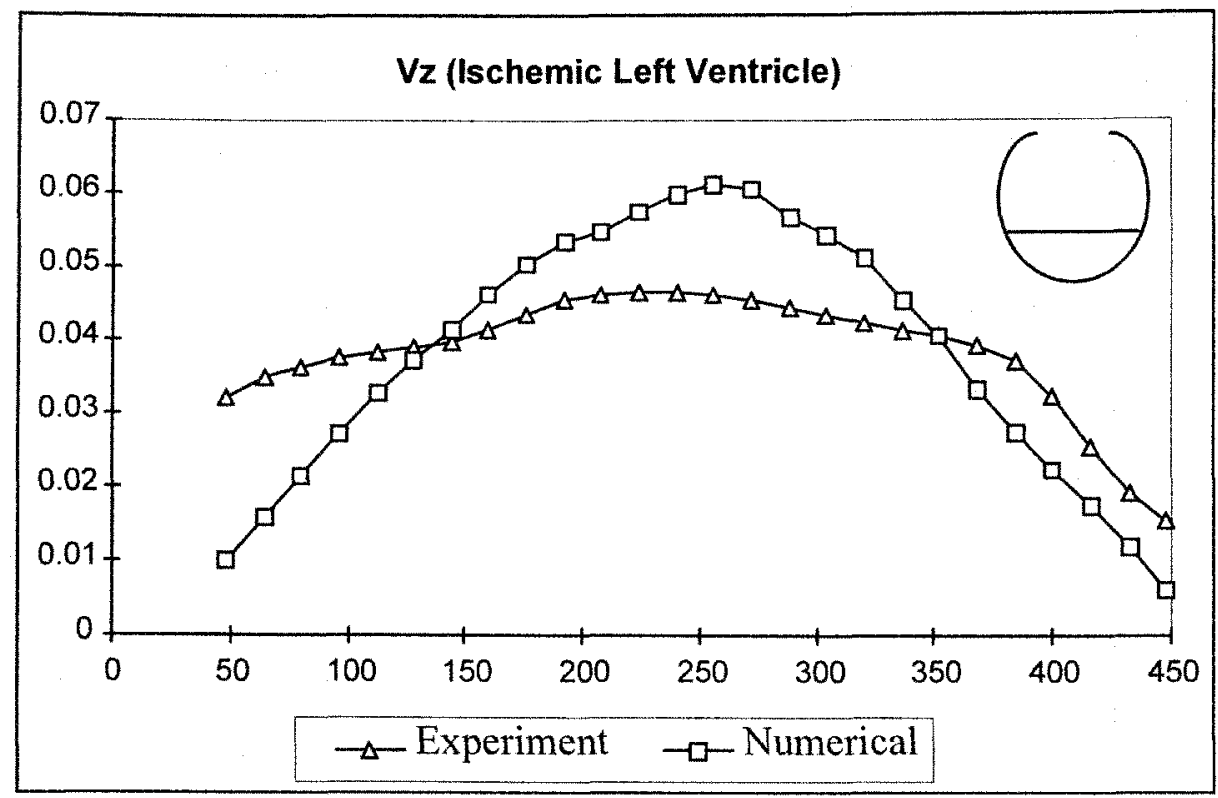

(a)

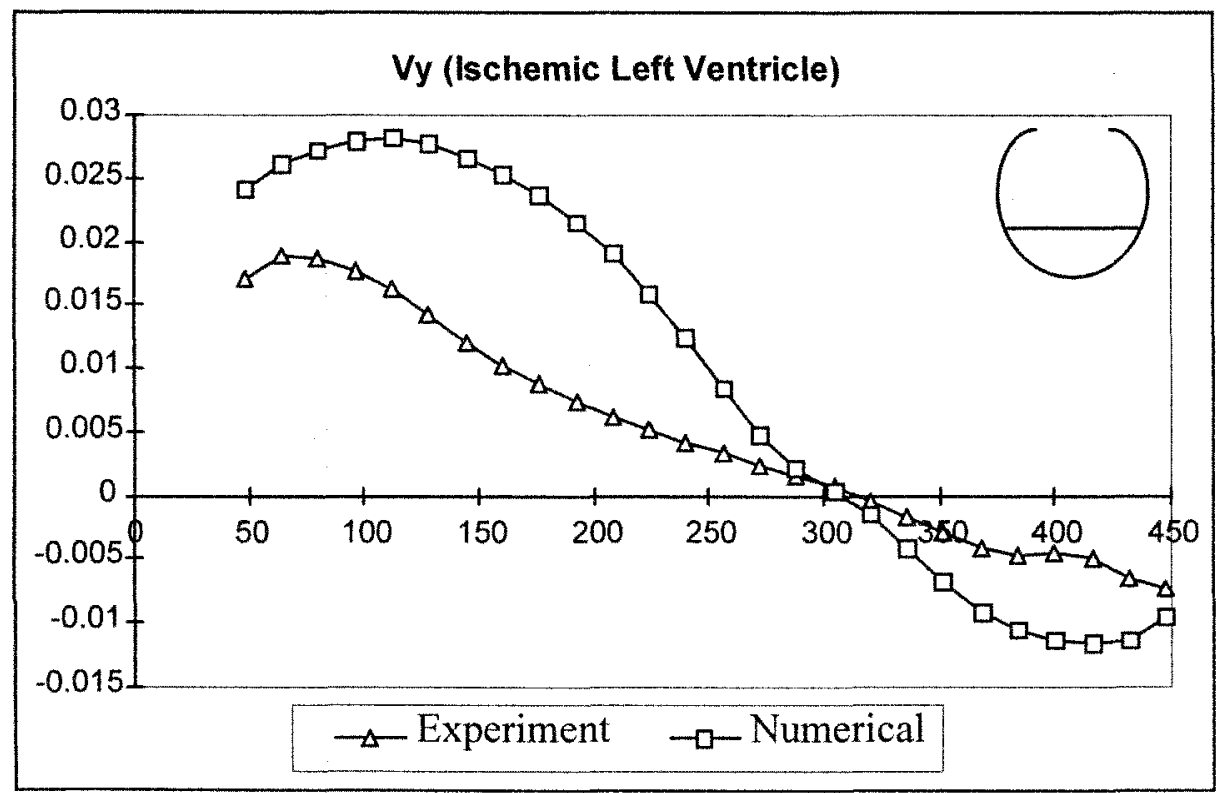

(b)

Figure 4.19 Velocity profile of experimental and numerical results close to bottom
(a) $\mathrm{Z}$ component
(b) Y component 


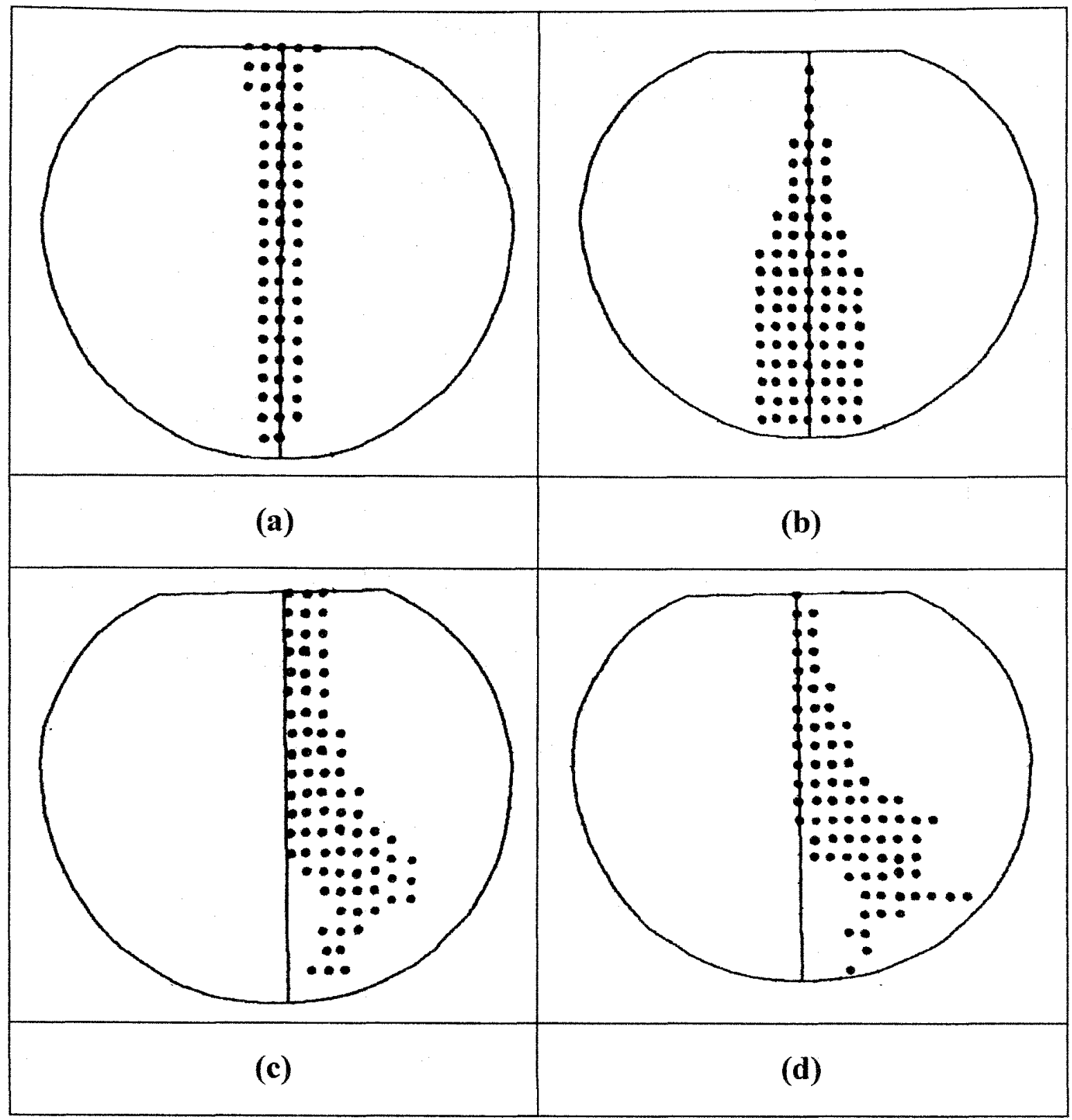

Figure 4.20 Comparison of the CER of experimental and numerical results
(a) numerical normal LV
(b) experimental normal LV
(c) numerical ischemic LV
(d) experimental ischemic LV 


\section{CHAPTER V}

\section{CONCLUSIONS AND RECOMMENDATIONS}

\section{FOR THE FUTURE WORK}

\section{V.1 Conclusion}

The present work is a continuation of the left ventricular flow dynamics study. The main objective was to explore the validity and efficacy of the CER and the CER coefficient as a new diagnostic tool of ischemic heart disease based on a physiologic shaped three-dimensional model, and to obtain a direct qualitative and quantitative validation of the numerical model with experimental measurements.

As in the previous models, the CER appears to be a useful tool to visualize changing flow pattern with changes in wall motion resulting from changes in the severity of the simulated ischemia. For the hypokinesis cases, the CER was able to indicate the approximate position of the ischemia boundary. With the same ischemic region, the CER coefficient was sensitive to the severity of the ischemia. Both the CER and the CER coefficient are more sensitive to degrees of hypokinesis than to degrees of dyskinesis. 
In the previous two-dimensional model, a large CER was considered better. In the current model, a good CER has the appearance of a solid pillar shaped and resides in the middle of the left ventricular cavity. When the CER increased in size in one direction, it would most likely change into a thin flat shape, which corresponding to the wall motion modeled with mild hypokinesis.

Compared to the previous two-dimensional model and threedimensional spherical shaped model, the current model most resembles the geometry of the left ventricle. The current study also included a complete detailed spatial observation of the CER as well as its relationship to the simulated ischemic wall motion. Some new phenomenon in the flow patterns and the CER are observed, which were impossible to observe in the two-dimensional model and were not observed in a spherical shaped threedimensional model. This further provides the necessity for building a threedimensional, real shaped model, which closely resembles the physiological geometry.

Another advantage of this model over the other three-dimensional models is the fact that the boundary conditions are obtained from the left ventricular wall motion. 
The main limitation of the model is the steady state assumption. The contraction of the left ventricle is a transient continuous motion, as well as the resulting flow. However, our current computational resources do not allow a transient computation.

The comparison of the numerical and experimental results gave a fairly good correlation. The experimental results validate the adequacy of the numerical model to predict left ventricular velocity vector fields based on computed wall motion.

\section{V.2 Recommendations for future work}

Although the numerical model used in the current study is a lot closer to the real left ventricle compared with other models, it's still symmetric with respect to the $\mathrm{Y}-\mathrm{Z}$ plane, and the reconstruction method utilized in the current study is very simple. There are many groups working on the threedimensional reconstruction methodology which is complicated yet more accurate. In the future, we should collaborate with other groups in order to build our model with a more accurate geometry.

The governing equation utilized in the current study is for steady state flow, which is not true for the real left ventricular flow. The main reason 
we didn't used the transient simulation as in the two dimensional model is the limitation of the computer resources. In the future, it is hopeful that the transient simulation will be performed.

From the comparison of the experimental and numerical results, we can clearly see the difference between the free surface boundary condition and the quasi-steady inflow we used in the current study. A model of the free surface boundary condition is necessary for the future study.

The current definition of the CER only takes the velocity direction into account. From the comparison of the flow patterns of the model with small ischemia and larger ischemia, the magnitude of the velocity also plays an important role. When eventually the CER is used as a diagnostic index for ischemic heart disease, it should be able to take the flow magnitude into account as well. 


\section{REFERENCES}

1. Adrian, R. J., 1991, "Particle-Imaging Techniques for Experimental Fluid Mechanics", Annu. Rev. Fluid Mech. 23:305-39.

2. Georgiadis, J. G., Wang, M. and Pasipoularides, A., 1992, "Computational Fluid Dynamics of Left Ventricular Ejection", Annals of Biomedical Engineering, 20:81-97.

3. Gonzalez, E. and Schoephoerster, R. T. , 1996, "A Simulation of Three-Dimensional Systolic Flow Dynamics in a Spherical Ventricle: Effects of Abnormal Wall Motion", Annals of Biomedical Engineering, 24:48-57.

4. Gonzalez, E., 1994, "Development of a Three-Dimensional Model of Left Ventricular Flow Dynamics", Master Thesis, Florida International University.

5. Green, J. F., 1987, Fundemental Cardiovascular and Pulmonary Physiology, Second Edition, Philadelphia.

6. Guyton, C., 1991, Textbook of Medical Physiology, Eighth Edition, Philadelphia.

7. Hampton, T., Shim, Y. and Pasipoularides, A, 1992, "Finite Element Analysis of Cardac Ejection Dynamics: Ultrasonic Implications", Advances in Bioengineering, ASME, 22:371-374.

8. Katz, Arnold M., 1977, Physiology of the Heart, Raven Press, New York.

9. McQueen, D. M. and Peskin, C. S., 1988, "A Three-Dimensional Computational Method for Blood Flow in the Heart. II. Contractile Fibers", Journal of Computational Physics, 82:298-297. 
10. Hashimoto, T., Ohte, N. and Narita, H., 1992, "Evaluation of a New Systolic Time Intrval, the Q-V Peak: Effects of heart Rate, Contractile State, and loading Conditions in Dogs", Angiology, 43: 685.

11. Ohte, N., Hashimoto, T and Narita, H., 1990, "Noninvasive Evaluation of Left Ventricular Performance with New Systolic Time Interval, the Q-V Peak, and Comparison with Established Systolic Time Intervals", The American Journam of Cardiology, 66:1018.

12. Peskin, C. S. and McQueen, D. M., 1988, "A Three-Dimensional Computational Method for Blood Flow in the Heart. I. Immersed Elastic Fibers in a Viscous Incompressible Fluid", Journal of Computational Physics, 82:372-405.

13. Peskin, C. S., 1977, "Numerical Analysis of Blood Flow in the Heart Method", Journal of Computational Physics, 25:220-252.

14. Redaelli, A. and Montevecchi, F. M., 1996, "Computational Evaluation of Intraventricular Pressure Gradients Based on a Fluid-Structure Approach", Journam of Biomechanical Engineering, 118:529-538.

15. Schoephoerster, R. T., Silva, C. L. and Ray, G., 1994, "Evaluation of Left Ventricular Function Based on Simulated Flow Dynamics Computed rom Regional Wall Motion", Journam of Biomechanics, 27:125-136.

16. Schoephoerster, R. T., Silva, C. L. and Ray, G., 1993, "Finite Analytic Model for Left Ventricular Systolic Flow Dynamics", Journal of Engineering Mechanics, 119:733-747.

17. Schoephoerster, R. T. and Chandran, K. B., 1991, "Velocity and Turbulence Measurements Past Mitral Valve Prostheses in a Model Left Ventricle", Biomechanics, 24:549-562. 
18. Schoephoerster, R. T., and Ray, G., 1991, "Assessment of Left Ventricular Flow Dynamics Using the Finite Analytic Method: Potential for Clinical Applications", Mechanics Computing in 1990's and Beyond, ASCE, 1:524-528.

19. Silva, C. L., 1991, "A Numerical Model of Systolic Flow Dynamics for Normal and Abnormal Left Ventricular Wall Motion", Master Thesis, Florida International University.

20. Song, S. M. and Leahy, R. M., 1991, "Computation of 3-D Velocity Fields from 3-D Cine CT Images of a Human Heart", IEEE Transactions on Medical Imaging, 10:295-306.

21. Taylor,T. W. and Yamaguchi, T., 1995, "Realistic Three-Dimensional Left Ventricular Ejection Determined from Computational Fluid Dynamics", Med. Eng. Phys., 17:602-608.

22. Taylor, T. W., Okino, H. and Yamaguchi, T.,"Three-Dimensional Analysis of Left Ventricular Ejection Using Computational Fluid Dynamics", Journal of Biomechanical Engineering, 116:127-130, 1994.

23. The American Heart Association, 1977, "Heartbook", E. P. Dutton, New York.

24. Walker, P. G., Cranney, G. B., Grimes, R. Y., Delatore, J., Rectenwald, J., Pohost, G. M. and Yoganathan, A. P., 1996, "Three-Dimensional Reconstruction of the Flow in a Human Left Heart by Using Magnetic Resonance Phase Velocity Encoding", Annals of Biomedical Engineering, 24:139-147.

25. Wendt, J.F., 1992, Computational Fluid Mechanics, Springer-Verlag, New York.

26. Willert, C. E. and Gharib, M., 1991, "Digital Particle Image Velocimetry", Experiments in Fluids, 10:181-193. 
27. Yoganathan, A. P., Lemmon Jr., J. D., Kim,, Y. H., Levine, R. A. and Vesier, C. C., 1995, "A Three-Dimensional Computational Investigation of Intraventricular Fluid Dynamics: Examination into the Initiation of Systolic Anterior Motion of the Mitral Valve Leaflets", Transactions of the ASME, 177:94-102.

28. Yoganathan, A. P., Lemmon Jr., J. D., Kim, Y. H., Walker, P. G., Levine, R. A. and Vesier, C. C., 1994, "A Computatonal Study of a Thin-Walled Three-Dimensional Left Ventricle During Early Systole", Journal of Biomechanical Engineering, 116:307-314. 


\section{APPENDIX A}

/*Prograin Geo_Gen.C

Language: $\mathrm{C}$

This program generate the three dimensional geometry

data from the two-dimensional data

*/

\#include $<$ stdio.h $>$

\#include $<$ math.h $>$

\#define N 5000

\#define N1 $200 / *$ \# of slcies along the long axis*/

\#define N2 $200 / *$ \# of points of each slice*/

\#define PI 3.1415926

\#define R $0.0043271 / *$ demensionalizing coefficient*/

struct POINT\{

double $\mathrm{x}$;

double y;

double $\mathrm{z}$;

int flag;

\} $\mathrm{CO}[\mathrm{N} 1+1][\mathrm{N} 2+1]$;

main()

\{double $\mathrm{X}[\mathrm{N}], \mathrm{Y}[\mathrm{N}]$;

int $\mathrm{i}, \mathrm{j}, \mathrm{n} ; /^{*} \mathrm{n}$ is total points in eash orgional 2-D data file, like of lvh.t0-5.

It may be different for different data file */

double $\mathrm{a}, \mathrm{b} ; /^{*} \mathrm{x}$ y coord of center of outlet*/

int $\mathrm{I} ; I^{*}$ point \# of the lowest point*/

double b1,LX[N1+1],LY[N1+1],RX[N1+1],RY[N1+1];

double b2,lx[N1+1],ly[N1+1],rx[N1+1],ry[N1+1];

double temp;

char stemp[50];

FILE *fp, ${ }^{*}$ pr;

double $r$,alfa,delta,xtemp,ytemp,ztemp;

double a_outlet,b_outlet; /*center of the outlet*/ 
for $(\mathrm{i}=1 ; \mathrm{i}<=\mathrm{N} 1 ; \mathrm{i}++)$

for $(j=1 ; j<=N 2 ; j++)$

$\mathrm{CO}[\mathrm{i}][\mathrm{j}]$.flag $=0$;

$\mathrm{fp}=$ fopen("lvs.t4","r"); /*Read in 2-D origional data*/

$\mathrm{i}=0$;

do \{

$\mathrm{i}=\mathrm{i}+1$;

fscanf(fp, "\%lf,\%lf",\&X[i],\&Y[i]);

$X[i]=-X[i]$;

\} while $(\mathrm{feof}(\mathrm{fp})==0)$;

fclose(fp);

$\mathrm{n}=\mathrm{i}-1$;

a_outlet $=(\mathrm{X}[1]+\mathrm{X}[\mathrm{n}]) / 2 ; / *$ Caculate outlet center $* /$

b_outlet $=(\mathrm{Y}[1]+\mathrm{Y}[\mathrm{n}]) / 2$;

$\mathrm{b} \overline{1}=99999$;

for $(\mathrm{i}=1 ; \mathrm{i}<=\mathrm{n} ; \mathrm{i}++) / *$ Searching for the Apex point*/

$\{\operatorname{if}(\mathrm{bl}>=\mathrm{Y}[\mathrm{i}])$

$\{\mathrm{b} 1=\mathrm{Y}[\mathrm{i}]$;

$\mathrm{I}=\mathrm{i}$;

\}

\}

$\mathrm{fp}=$ fopen("s4.L","r"); /*Read in seperation points on left and right halves.*/ pr=fopen("s4.R","r"); /*s4.L and s4.R are output file segments from FIDAP*/ for $(\mathrm{i}=1 ; \mathrm{i}<=\mathrm{N} 1+1 ; \mathrm{i}++)$

\{

for $(\mathrm{j}=1 ; \mathrm{j}<=24 ; \mathrm{j}++)$

fscanf(fp,"\%s",\&stemp);

fscanf(fp, \%c \%c",\&stemp[1],\&stemp[2]);

if $(i<=2)$

fscanf(fp,"\%lf, \%lf,\%lf",\&LX[i],\&LY[i],\&temp); 
if $(i>2)$

fscanf(fp,"\%lf, \%lf,\%lf",\&LX[i-1],\&LY[i-1],\&temp);

fscanf(fp,"\%c",\&stemp[1]);

for $(\mathrm{j}=1 ; \mathrm{j}<=24 ; \mathrm{j}++)$

fscanf(pr, "\%s",\&stemp);

fscanf(pr,"\%c \%c",\&stemp[1],\&stemp[2]);

if $(i<=2)$

fscanf(pr,"\%lf, \%lf,\%lf",\&RX[i],\&RY[i],\&temp);

if $(i>2)$

fscanf(pr,"\%lf, \%lf,\%lf",\&RX[i-1],\&RY[i-1],\&temp);

fscanf(pr,"\%c",\&stemp[1]);

\}

$\operatorname{lx}[1]=\mathrm{LX}[1] ; \operatorname{ly}[1]=\mathrm{LY}[1]$

$\operatorname{rx}[1]=\mathrm{RX}[1] ; \operatorname{ry}[1]=\mathrm{RY}[1]$;

for $(\mathrm{i}=2 ; \mathrm{i}<=\mathrm{N} 1 ; \mathrm{i}++)$

$\{\mathrm{b} 1=99999 * 99999$;

for $(j=2 ; j<I ; j++)$

$\{$ temp $=(X[j]-L X[i]) *(X[j]-L X[i])+(Y[j]-L Y[i]) *(Y[j]-L Y[i])$;

if (temp $<b 1)$

$\{\mathrm{b} 1=$ temp;

$\mathrm{lx}[\mathrm{i}]=\mathrm{X}[\mathrm{j}]$;

$\mathrm{ly}[\mathrm{i}]=\mathrm{Y}[\mathrm{j}]$;

\}

\}

b2=99999*99999.;

for $(\mathrm{j}=\mathrm{I}+1 ; \mathrm{j}<\mathrm{n} ; \mathrm{j}++)$

$\{$ temp $=(X[j]-R X[i]) *(X[j]-R X[i])+(Y[j]-R Y[i]) *(Y[j]-R Y[i])$;

if (temp $<\mathrm{b} 2)$

\{b2=temp;

$\operatorname{rx}[\mathrm{i}]=\mathrm{X}[\mathrm{j}]$;

$\mathrm{ry}[\mathrm{i}]=\mathrm{Y}[\mathrm{j}]$;

\}

\} 
for $(\mathrm{i}=1 ; \mathrm{i}<=\mathrm{N} 1 ; \mathrm{i}++) / *$ rotate each segment, get the points on each tilted circle $* /$ $\{\mathrm{a}=(\mathrm{lx}[\mathrm{i}]+\mathrm{rx}[\mathrm{i}]) / 2 ; / *$ center of the segment, also rotate center $* /$ $\mathrm{b}=(\mathrm{ly}[\mathrm{i}]+\mathrm{ry}[\mathrm{i}]) / 2$;

$\mathrm{r}=\operatorname{sqrt}((\mathrm{lx}[\mathrm{i}]-\mathrm{rx}[\mathrm{i}]) *(\operatorname{lx}[\mathrm{i}]-\mathrm{rx}[\mathrm{i}])+(\operatorname{ly}[\mathrm{i}]-\mathrm{ry}[\mathrm{i}]) *(\operatorname{ly}[\mathrm{i}]-\mathrm{ry}[\mathrm{i}])) / 2$;

alfa $=\operatorname{atan}((\operatorname{ry}[\mathrm{i}]-\mathrm{ly}[\mathrm{i}]) /(\operatorname{rx}[\mathrm{i}]-\mathrm{lx}[\mathrm{i}])) ; / *$ Angle to rotate*/

delta $=2 * \mathrm{PI} / \mathrm{N} 2$

for $(\mathrm{j}=1 ; \mathrm{j}<=\mathrm{N} 2 ; \mathrm{j}++)$

$\{\mathrm{xtemp}=\mathrm{r} * \cos (\operatorname{delta} *(j-1))$;

ytemp $=r^{*} \sin \left(\operatorname{delta}^{*}(j-1)\right)$;

ztemp $=0$;

if $\left(((\operatorname{delta} *(j-1))>=P I / 4) \& \&\left((\operatorname{delta} *(j-1))<=P I^{*} 3 / 4\right)\right)$

$\mathrm{CO}[\mathrm{i}][\mathrm{j}]$. flag $=1$;

$\mathrm{CO}[\mathrm{i}][\mathrm{j}] \cdot \mathrm{x}=(\mathrm{xtemp}) * \mathrm{R}$

$\mathrm{CO}[\mathrm{i}][\mathrm{j}] . \mathrm{y}=(\mathrm{a}-\mathrm{ap}+\mathrm{ytemp} * \cos (\text { alfa })-z \operatorname{temp} * \sin (\mathrm{alfa}))^{*} \mathrm{R}$;

$\mathrm{CO}[\mathrm{i}][\mathrm{j}] \cdot \mathrm{z}=\left(\mathrm{b}-\mathrm{bp}+\mathrm{ytemp} * \sin (\mathrm{alfa})+\mathrm{ztemp}{ }^{*} \cos (\mathrm{alfa})\right)^{*} \mathrm{R}$;

\}$^{3}$

$1 *$ output to screen the 3-D geometry data. It can be directed to any file*/ printf("0 \%lf \%lf 1 ln",(X[I]-ap)*R,(Y[I]-bp)*R);

for $(\mathrm{i}=1 ; \mathrm{i}<=\mathrm{N} 1 ; \mathrm{i}++)$

$\{$ for $(\mathrm{j}=1 ; \mathrm{j}<=\mathrm{N} 2 ; \mathrm{j}++)$

printf("\%lf \%lf \%lf \%dln",CO[i][j].x,CO[i][j].y,CO[i][j].z,CO[i][j].flag);

\}

\}

/* End of the Program*/ 
/* Fpoints_Gen.c

This program is the newer version of the previous program. Besides generates the 3-D geometry data, it also generates the FIDAP input file for all the points of each geometry with the input of the geometry data file *1

\#include $<$ stdio.h $>$

\#include $<$ math.h $>$

\#define N 5000

\#define N1 $30 / *$ \# of slcies along the long axis */

\#define N2 $16 / *$ \# of points of each slice */

\#defime PI 3.1415926

\#defime R 0.0043271 /*dimensionalize coefficient */

struct POINT \{

double $\mathrm{x}$;

double y;

double $\mathrm{z}$;

int flag;

\} $\mathrm{CO}[\mathrm{N} 1+10][\mathrm{N} 2+10]$;

struct out_point \{

double $\mathrm{x}$;

double y;

double $\mathrm{z}$;

\}out_temp[10],out_p1[10],out_p3[10],out_p6[10],out_p15[10],out_p24[10];

main()

\{double $\mathrm{X}[\mathrm{N}], \mathrm{Y}[\mathrm{N}]$;

int $\mathrm{i}, \mathrm{j}, \mathrm{n} ; /^{*} \mathrm{n}$ is total points of lvh.t0-5*/

double $\mathrm{a}, \mathrm{b} ; / *$ coord of center of each slice*/

int $\mathrm{I} ; \mathrm{I}^{*}$ point \# of the lowest point*/

double b1,LX[N1+10],LY[N1+10],RX[N1+10],RY[N1+10];

double b2, $\operatorname{lx}[\mathrm{N} 1+10], \operatorname{ly}[\mathrm{N} 1+10], \mathrm{rx}[\mathrm{N} 1+10], \mathrm{ry}[\mathrm{N} 1+10]$;

double temp;

char stemp[50];

FILE *fp, ${ }^{*}$ pr; 
double r,alfa,delta,xtemp,ytemp,ztemp;

double ap,bp; /*center of the outlet*

double $\mathrm{X} 0, \mathrm{Y} 0, \mathrm{Z} 0, \mathrm{r} 0$;

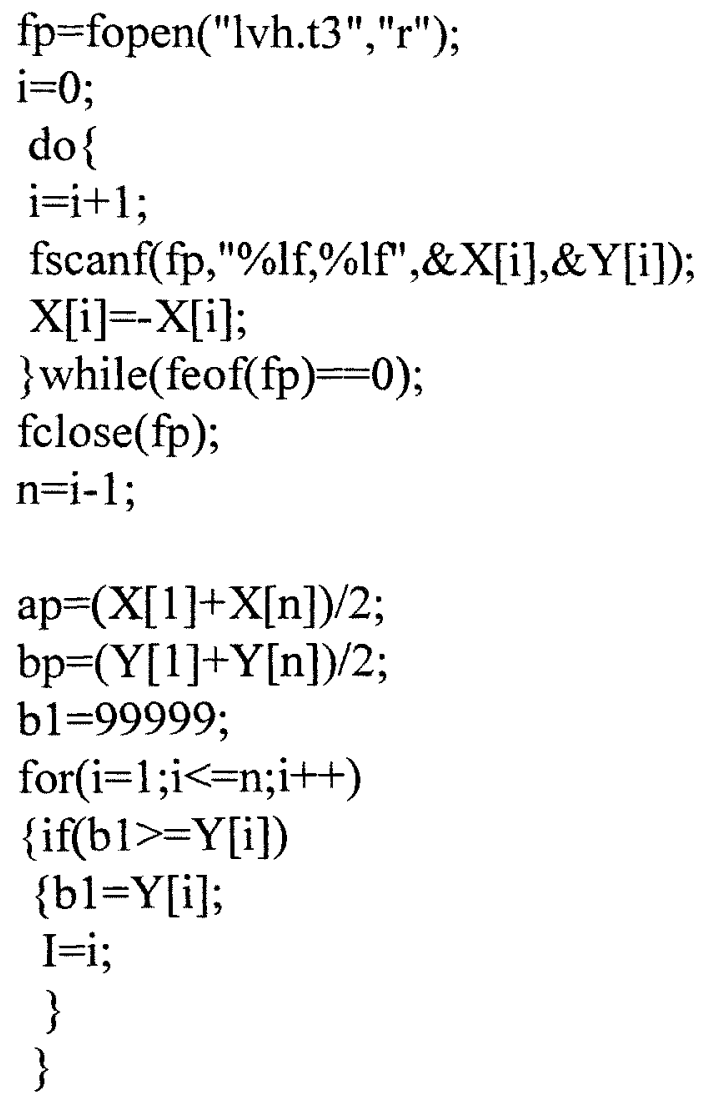


fscanf(fp,"\%c",\&stemp[1]);

for $(\mathrm{j}=1 ; \mathrm{j}<=24 ; \mathrm{j}++)$

fscanf(pr,"\%s",\&stemp);

fscanf(pr,"\%c \%c",\&stemp[1],\&stemp[2]);

if $(\mathrm{i}<=2)$

fscanf(pr,"\%lf, \%lf,\%lf",\&RX[i],\&RY[i],\&temp);

if $(\mathrm{i}>2)$

fscanf(pr,"\%lf, \%lf,\%lf",\&RX[i-1],\&RY[i-1],\&temp);

fscanf(pr,"\%c",\&stemp[1]);

\}

$\operatorname{lx}[1]=\mathrm{LX}[1] ; \mathrm{ly}[1]=\mathrm{LY}[1]$

$\operatorname{rx}[1]=\mathrm{RX}[1] ; \operatorname{ry}[1]=\mathrm{RY}[1] ;$

for $(\mathrm{i}=2 ; \mathrm{i}<=\mathrm{N} 1 ; \mathrm{i}++)$

$\{\mathrm{b} 1=99999$;

for $(\mathrm{j}=2 ; \mathrm{j}<\mathrm{I} ; \mathrm{j}++)$

$\{$ temp $=(X[j]-L X[i]) *(X[j]-L X[i])+(Y[j]-L Y[i]) *(Y[j]-L Y[i])$;

if $($ temp $<$ b1)

$\{\mathrm{b} 1=$ temp;

$\mathrm{lx}[\mathrm{i}]=\mathrm{X}[\mathrm{j}]$;

$1 y[i]=Y[j] ;$

\}

\}

b2 $=99999$;

for $(\mathrm{j}=\mathrm{I}+1 ; \mathrm{j}<\mathrm{n} ; \mathrm{j}++)$

$\{$ temp $=(X[j]-R X[i]) *(X[j]-R X[i])+(Y[j]-R Y[i]) *(Y[j]-R Y[i])$;

if $($ temp $<$ b2)

$\{\mathrm{b} 2=$ temp;

$\mathrm{rx}[\mathrm{i}]=\mathrm{X}[\mathrm{j}]$;

$\operatorname{ry}[\mathrm{i}]=\mathrm{Y}[\mathrm{j}]$

\}

\}

\} 
for $(\mathrm{i}=1 ; \mathrm{i}<=\mathrm{N} 1 ; \mathrm{i}++)$

$\{\mathrm{a}=(\mathrm{lx}[\mathrm{i}]+\mathrm{rx}[\mathrm{i}]) / 2$;

$\mathrm{b}=(\mathrm{ly}[\mathrm{i}]+\mathrm{ry}[\mathrm{i}]) / 2$;

$\mathrm{r}=\operatorname{sqrt}((\mathrm{lx}[\mathrm{i}]-\mathrm{rx}[\mathrm{i}]) *(\mathrm{~lx}[\mathrm{i}]-\mathrm{rx}[\mathrm{i}])+(\mathrm{ly}[\mathrm{i}]-\mathrm{ry}[\mathrm{i}]) *(\mathrm{ly}[\mathrm{i}]-\mathrm{ry}[\mathrm{i}])) / 2$;

alfa $=\operatorname{atan}((\operatorname{ry}[\mathrm{i}]-\mathrm{ly}[\mathrm{i}]) /(\operatorname{rx}[\mathrm{i}]-\mathrm{lx}[\mathrm{i}]))$;

delta $=2 * \mathrm{PI} / \mathrm{N} 2$;

for $(j=1 ; j<=N 2 ; j++)$

$\left\{\mathrm{xtemp}=\mathrm{r}^{*} \cos (\operatorname{delta} *(\mathrm{j}-1))\right.$;

ytemp $=r^{*} \sin (\operatorname{delta*}(j-1))$;

ztemp $=0$;

if $(($ delta $>=P I / 4) \& \&($ delta $<=P I * 3 / 4))$

$\mathrm{CO}[\mathrm{i}][\mathrm{j}]$. flag $=1$;

$\mathrm{CO}[\mathrm{i}][\mathrm{j}] \cdot \mathrm{x}=(\mathrm{xtemp}) * \mathrm{R}$;

$\mathrm{CO}[\mathrm{i}][\mathrm{j}] . \mathrm{y}=\left(\mathrm{a}-\mathrm{ap}+\mathrm{ytemp} \mathrm{p}^{*} \cos (\mathrm{alfa})-z \text { temp* } \sin (\text { alfa })\right)^{*} \mathrm{R}$;

$\mathrm{CO}[\mathrm{i}][\mathrm{j}] . z=\left(b-b p+y t e m p * \sin (\text { alfa })+z \operatorname{tem} p^{*} \cos (\text { alfa })\right)^{*} \mathrm{R}$;

\}

$/^{*}$ output all the points of the cubic regions inside the control volume*/

if $(\mathrm{i}=1)$

\{out_temp[1].x $=0 ;$ out_temp[1].y $=0$; out_temp[1].z $=0$;

out temp[2]. $\mathrm{x}=\mathrm{r} / 2$; out temp[2]. $\mathrm{y}=\mathrm{r} / 2 ;$ out temp[1]. $\mathrm{z}=0$;

out temp[3].x=-r/2; out_temp[3].y=r/2; out_temp[1].z=0;

out_temp[4].x=-r/2; out_temp[4].y=-r/2; out temp[1].z=0;

out_temp[5].x=r/2; out_temp[5].y=-r/2; out_temp[1].z=0;

out temp[6].x=r/2; out temp[6].y=0; out temp $[1] . z=0$;

out_temp[7].x=0; out_temp[7].y=r/2; out_temp[1].z=0;

out_temp[8].x=-r/2; out_temp[8].y=0; out_temp[1].z=0;

out_temp[9].x=0; out_temp[9].y=-r/2; out_temp[1].z=0;

for $(j=1 ; j<=9 ; j++)$

\{out_pl[j].x=(out_temp[j].x $)^{*} \mathrm{R}$;

out_p1[j].y=(a-ap+out_temp[j].y*cos(alfa)-out_temp[j].z*sin(alfa) $)^{*} \mathrm{R}$; out_p1[j].z $=\left(b-b p+o u t \text { temp[j]. } y^{*} \sin (\text { alfa })+o u \text { t temp[j].z* } \cos (\text { alfa })\right)^{*} R$;

\} 
if $(\mathrm{i}==3)$

\{out_temp[1].x=0; out temp[1].y=0; out_temp[1].z=0;

out_temp[2]. $x=r / 2 ;$ out_temp[2].y $=r / 2 ;$ out_temp[1].z=0;

out_temp[3].x=-r/2; out_temp[3].y=r/2; out_temp[1].z=0;

out_temp[4].x=-r/2; out_temp[4].y=-r/2; out_temp[1].z=0;

out_temp [5].x=r/2; out_temp[5].y=-r/2; out_temp[1].z=0;

out_temp[6].x=r/2; out_temp[6].y=0; out_temp[1].z=0;

out_temp[7]. $x=0$; out_temp[7].y $=r / 2$; out temp[1].z=0;

out_temp[8]. $\mathrm{x}=-\mathrm{r} / 2$; out_temp[8]. $\mathrm{y}=0$; out_temp[1].z=0;

out temp[9].x $=0$; out_temp[9].y=-r/2; out_temp[1].z=0;

for $(j=1 ; j<=9 ; j++)$

\{out_p3[j].x=(out_temp[j].x)*R;

out_p3[j].y=(a-ap+out_temp[j].y*cos(alfa)-out_temp[j].z*sin(alfa $) * R$;

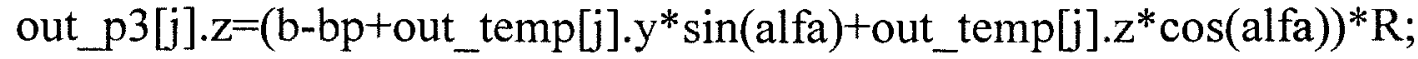

\}

\}

if $(\mathrm{i}==6)$

\{out_temp $[1] . x=0 ;$ out_temp $[1] . y=0 ;$ out_temp $[1] . z=0$;

out temp[2].x $=\mathrm{r} / 2$; out temp[2].y $=\mathrm{r} / 2 ;$ out_temp[1].z=0;

out temp[3]. $\mathrm{x}=-\mathrm{r} / 2$; out temp[3].y=r/2; out temp[1].z=0;

out temp [4].x =-r/2; out temp[4].y=-r/2; out temp[1].z=0;

out temp[5].x $=r / 2 ;$ out temp[5].y=-r/2; out_temp[1].z=0;

out_temp[6]. $\mathrm{x}=\mathrm{r} / 2 ;$ out_temp $[6] . \mathrm{y}=0 ;$ out_temp $[1] . \mathrm{z}=0$;

out_temp[7]. $\mathrm{x}=0$; out_temp[7].y=r/2; out_temp[1].z=0;

out_temp[8].x=-r/2; out_temp[8].y $=0$; out_temp[1].z=0;

out temp[9].x $=0$; out_temp[9].y=-r/2; out_temp[1].z=0;

for $(\mathrm{j}=1 ; \mathrm{j}<=9 ; \mathrm{j}++)$

\{out $\mathrm{p} 6[\mathrm{j}] \cdot \mathrm{x}=$ (out_temp[j].x)*R;

out_p6[j].y=(a-ap+out_temp[j].y*os(alfa)-out_temp[j].z*sin(alfa) $)^{*} \mathrm{R}$;

out_p6[i].z=(b-bp+out_temp[j].y*sin(alfa)+out_temp[j].z*cos(alfa) $)^{*} \mathrm{R}$;

\}$_{\text {if }(i==15)}^{\}}$

\{out_temp[1].x=0; out_temp[1].y=0; out_temp[1].z=0;

out_temp[2].x=r/2; out temp[2].y $=\mathrm{r} / 2$; out_temp[1].z=0;

out_temp[3].x $=-r / 2 ;$ out_temp[3].y $=r / 2$; out_temp[1].z=0; 


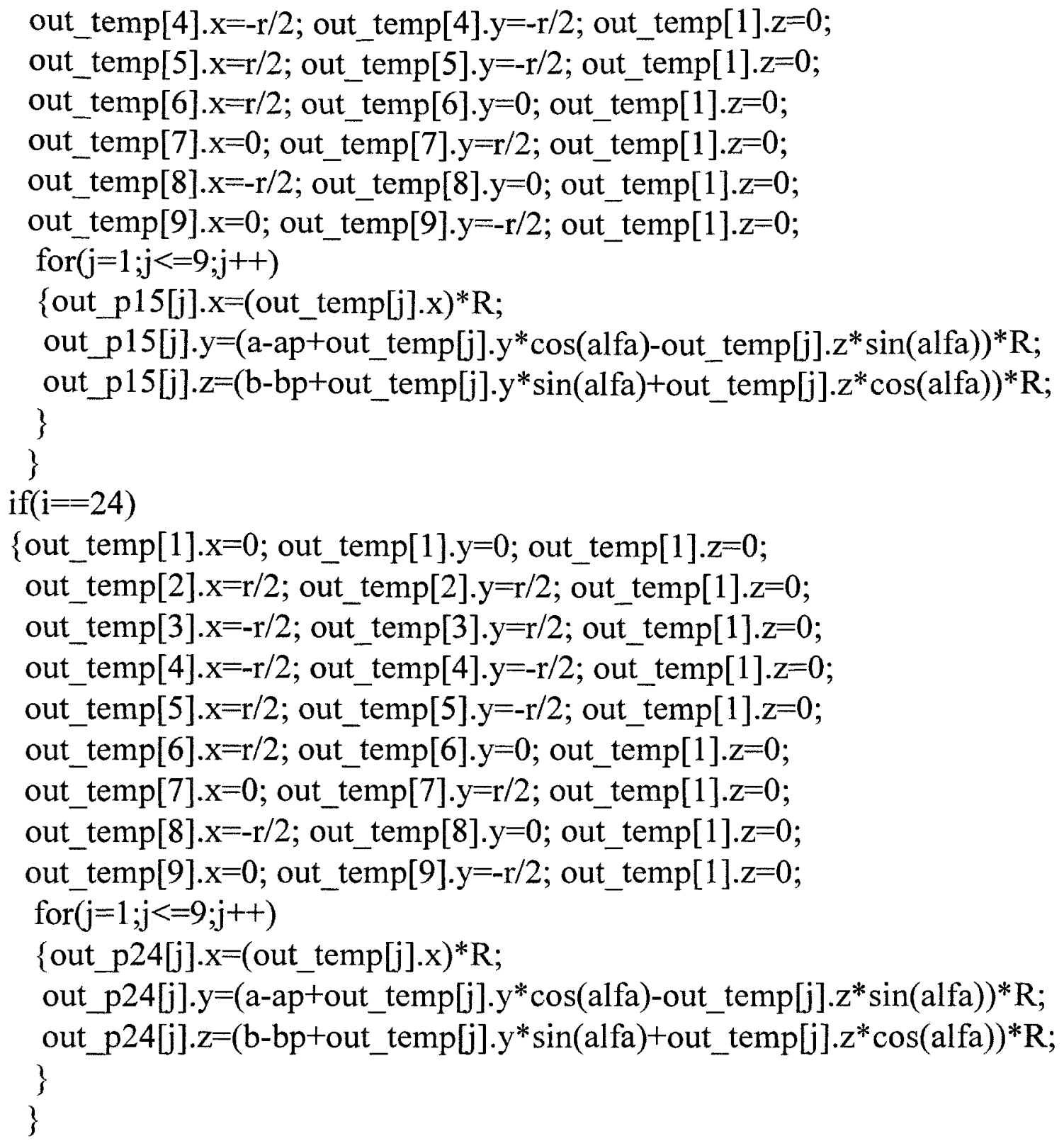

printf("FI-GEN( ELEM $=1, \mathrm{POIN}=1, \mathrm{CURV}=1, \mathrm{SURF}=1, \mathrm{NODE}=0$, $\mathrm{MEDG}=1, \mathrm{MLOO}=1, \mathrm{MFAC}=1, \mathrm{BEDG}=1, \mathrm{SPAV}=1, \mathrm{MSHE}=1, \mathrm{MSOL}$ $\left.=1) \backslash n^{\prime \prime}\right)$ 
printf("POINT( ADD, VISI, CART, COOR, $X=0, Y=\% l f, Z=\% 1 f) \backslash n ",(X[I]-$ ap)*R,(Y[I]-bp)*R);

for $(j=1 ; j<=N 2 ; j=j+2)$

$\{$ for $(\mathrm{i}=1 ; \mathrm{i}<=\mathrm{N} 1 ; \mathrm{i}++)$

printf("POINT( ADD, VISI, CART, COOR, $\mathrm{X}=\% \mathrm{le}, \mathrm{Y}=\% \mathrm{le}, \mathrm{Z}=$ $\%$ le) $\backslash n ", \mathrm{CO}[\mathrm{i}][\mathrm{j}] . \mathrm{x}, \mathrm{CO}[\mathrm{i}][\mathrm{j}] . \mathrm{y}, \mathrm{CO}[\mathrm{i}][\mathrm{j}] . z)$;

\}

$\operatorname{printf}(" \wedge n ")$;

for $(\mathrm{i}=2 ; \mathrm{i}<=\mathrm{N} 2 ; \mathrm{i}=\mathrm{i}+2)$

printf("POINT( ADD, VISI,CART,COOR, $X=\%$ le, $Y=\%$ le, $Z=$ $\%$ le) $\backslash n ", \mathrm{CO}[25][\mathrm{i}] . \mathrm{x}, \mathrm{CO}[25][\mathrm{i}] . \mathrm{y}, \mathrm{CO}[25][\mathrm{i}] . z)$;

for $(\mathrm{i}=1 ; \mathrm{i}<=5 ; \mathrm{i}++)$

printf("POINT( ADD, VISI,CART,COOR, X=\%le, $Y=\% 1 \mathrm{l}, \mathrm{Z}=$ \%le)ไn",out_p15[i].x,out_p15[i].y,out_p15[i].z);

for $(\mathrm{i}=1 ; \mathrm{i}<=5 ; \mathrm{i}++)$

printf("POINT( ADD, VISI,CART,COOR, X=\%le, Y =\%le, Z= $\%$ le)\n",out_p24[i].x,out_p24[i].y,out_p24[i].z);

for $(\mathrm{i}=1 ; \mathrm{i}<=5 ; \mathrm{i}++)$

printf("POINT( ADD, VISI,CART,COOR, X=\%le, Y =\%le, Z= $\%$ le)\n",out_p6[i].x,out_p6[i].y,out_p6[i].z);

for $(\mathrm{i}=6 ; \mathrm{i}<=9 ; \mathrm{i}++)$

printf("POINT( ADD, VISI,CART,COOR, $X=\%$ le, $Y=\%$ le, $Z=$ $\%$ le)ไn",out_p15[i].x,out_p15[i].y,out_p15[i].z);

for $(\mathrm{i}=6 ; \mathrm{i}<=9 ; \mathrm{i}++)$

printf("POINT( ADD, VISI,CART,COOR, $X=\% 1$, $Y=\% 1$, $Z=$ $\%$ le) $\backslash n$ ",out_p24[i].x,out_p24[i].y,out_p24[i].z);

for $(\mathrm{i}=6 ; \mathrm{i}<=9 ; \mathrm{i}++)$ 
printf("POINT( ADD, VISI,CART,COOR, X=\%le, Y =\%le, Z= \%le)\n",out_p6[i].x,out_p6[i].y,out_p6[i].z);

for $(\mathrm{i}=1 ; \mathrm{i}<=5 ; \mathrm{i}++)$

printf("POINT( ADD, VISI,CART,COOR, $\mathrm{X}=\% \mathrm{le}, \mathrm{Y}=\% \mathrm{le}, \mathrm{Z}=$ $\%$ le)\n",out_p3[i].x,out_p3[i].y,out_p3[i].z);

for $(\mathrm{i}=1 ; \mathrm{i}<=5 ; \mathrm{i}++)$

printf("POINT( ADD, VISI,CART,COOR, $X=\%$ le, $Y=\%$ le, $Z=$ $\%$ le)ไn",out_p1[i].x,out_p1[i].y,out_p1[i].z);

for $(\mathrm{i}=6 ; \mathrm{i}<=9 ; \mathrm{i}++)$

printf("POINT( ADD, VISI,CART,COOR, $X=\%$ le, $Y=\% l e, Z=$ \%le) (n",out_p3[i].x,out_p3[i].y,out_p3[i].z);

for $(\mathrm{i}=6 ; \mathrm{i}<=9 ; \mathrm{i}++)$

printf("POINT( ADD, VISI,CART,COOR, $X=\%$ le, $Y=\% 1$, $Z=$ $\%$ le)ไn",out_p1[i].x,out_p1[i].y,out_p1[i].z);

\}

$/ *$ End of the program*/ 


\section{APPENDIX B}

/*Program BCondition. C

Language: $\mathrm{C}$

This program caculates the velocity boundary conditions for natural and simulated ischemic LVs, and outputs the BCs in FIDAP input file format. */

\#include $<$ stdio.h $>$

\#include $<$ math.h $>$

\#define N 2000

\#define M 40100

\#define $\mathrm{n} 11561$

\#define PI 3.1415926535

\#define flag 1

main()

\{double $x[N], y[N], z[N], v x[N], v y[N], v z[N]$;

double lenth,minl,px[M],py[M],pz[M],U;

char s[100],sl;

FILE *fp;

long int $i, j, n, m, n p$, int 1 ,int2, int3;

int nu;

double length,11,alfa1,alfa2, beta1, beta2,alfa, beta;

fp=fopen("DAT","r"); /*DAT is the output file from FIDAP, contains the coordinates of all nodes on the surface*/

for $(\mathrm{i}=1 ; \mathrm{i}<=473 ; \mathrm{i}++)$

$\{$ for $(\mathrm{j}=1 ; \mathrm{j}<=5 ; \mathrm{j}++)$

fscanf(fp, $\%$ s",\&s);

fscanf(fp," \%d",\&nu);

for $(j=1 ; j<=31 ; j++)$

fscanf(fp, $\%$ s",\&s);

fscanf(fp," \%c",\&s1);

fscanf(fp,"\%lf,\%lf, \%lf",\&x[nu],\&y[nu],\&z[nu]);

fscanf(fp, "\%s",\&s); 
for $(\mathrm{i}=474 ; \mathrm{i}<=\mathrm{n} 1 ; \mathrm{i}++)$

$\{$ for $(j=1 ; j<=5 ; j++)$

fscanf(fp, "\%s",\&s);

fscanf(fp," \%d",\&nu);

for $(j=1 ; j<=25 ; j++)$

fscanf(fp,"\%s",\&s);

fscanf(fp," \%c",\&s1);

fscanf(fp, "\%lf,\%lf,\%lf",\&x[nu],\&y[nu],\&z[nu]);

fscanf(fp,"\%s",\&s);

\}

fclose(fp);

fp=fopen("GEO","r");

for $(\mathrm{i}=1 ; \mathrm{i}<=40001 ; \mathrm{i}++)$

\{fscanf(fp,"\%lf \%lf \%lf \%d",\&px[i],\&py[i],\&pz[i],\&j);

\}

fclose(fp);

$\mathrm{n}=\mathrm{i}-1$;

for $(\mathrm{i}=1 ; \mathrm{i}<=\mathrm{n} 1 ; \mathrm{i}++)$

$\{$ if $(\mathrm{z}[\mathrm{i}]<-0.895545)$

$\{\operatorname{minl}=999999.0$;

for $(j=1 ; j<=n ; j+)$

$\{$ lenth $=(x[i]-p x[j]) *(x[i]-p x[j])+(y[i]-p y[j]) *(y[i]-p y[j])+(z[i]-p z[j]) *(z[i]-$ $\mathrm{pz}[\mathrm{j}])$;

if (lenth $<\operatorname{minl})$

$\{\min =$ lenth;

$\mathrm{np}=\mathrm{j}$;

\}

\}

$\operatorname{vx}[\mathrm{i}]=(\mathrm{px}[\mathrm{np}]-\mathrm{x}[\mathrm{i}]) /(0.067)$;

$v y[i]=(p y[n p]-y[i]) /(0.067)$;

$\mathrm{vz}[\mathrm{i}]=(\mathrm{pz}[\mathrm{np}]-\mathrm{z}[\mathrm{i}]) /(0.067)$;

\} 


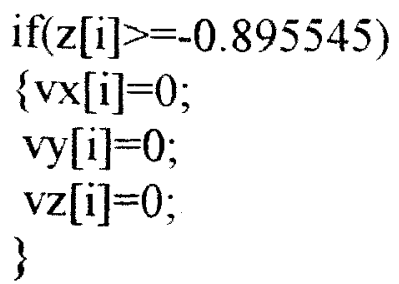

printf("BCNODE( ADD, VELO, NODE $=\% d$, CONS, X $=\%$ le, $Y=\%$ le, $Z=$ \%le )(n",i,vx[i],vy[i],vz[i]);

\}

\}

/*End of the Program*/ 